\title{
Automatic enhancement in 6D supergravity and F-theory models
}

\author{
Nikhil Raghuram, ${ }^{a}$ Washington Taylor $^{b}$ and Andrew P. Turner ${ }^{c}$ \\ ${ }^{a}$ Department of Physics, \\ Robeson Hall, 0435, Virginia Tech, 850 West Campus Drive, Blacksburg, VA 24061, U.S.A. \\ ${ }^{b}$ Center for Theoretical Physics, Department of Physics, \\ Massachusetts Institute of Technology, \\ 77 Massachusetts Avenue, Cambridge, MA 02139, U.S.A. \\ ${ }^{c}$ Department of Physics and Astronomy, University of Pennsylvania, \\ Philadelphia, PA 19104, U.S.A. \\ E-mail: nikhilr@vt.edu, wati@mit.edu, turnerap@sas.upenn.edu
}

\begin{abstract}
We observe that in many F-theory models, tuning a specific gauge group $G$ and matter content $M$ under certain circumstances leads to an automatic enhancement to a larger gauge group $G^{\prime} \supset G$ and matter content $M^{\prime} \supset M$. We propose that this is true for any theory $G, M$ whenever there exists a containing theory $G^{\prime}, M^{\prime}$ that cannot be Higgsed down to $G, M$. We give a number of examples including non-Higgsable gauge factors, nonabelian gauge factors, abelian gauge factors, and exotic matter. In each of these cases, tuning an F-theory model with the desired features produces either an enhancement or an inconsistency, often when the associated anomaly coefficient becomes too large. This principle applies to a variety of models in the apparent $6 \mathrm{D}$ supergravity swampland, including some of the simplest cases with $\mathrm{U}(1)$ and $\mathrm{SU}(N)$ gauge groups and generic matter, as well as infinite families of $\mathrm{U}(1)$ models with higher charges presented in the prior literature, potentially ruling out all these apparent swampland theories.
\end{abstract}

KEYwords: F-Theory, Gauge Symmetry, Supergravity Models

ArXiv EPrint: 2012.01437 


\section{Contents}

1 Introduction 1

2 Statement of the conjecture $\quad 3$

3 Review of 6D supergravity, F-theory realizations, and the swampland 4

3.1 6D supergravity theories and their realizations in F-theory 5

3.2 The 6D supergravity swampland 6

$\begin{array}{lll}\text { 3.2.1 Constraints on 6D supergravity theories } & 7\end{array}$

3.2.2 Apparent swampland for $T=0$, nonabelian $G$, and generic matter 8

3.2.3 Apparent swampland with abelian $G \quad 9$

$\begin{array}{ll}3.2 .4 & \text { Apparent swampland with } T>0\end{array}$

3.2.5 Apparent swampland with exotic matter for nonabelian $G \quad 11$

4 Nonabelian examples

$\begin{array}{lll}4.1 & \text { Non-Higgsable clusters } & 11\end{array}$

$\begin{array}{lll}4.2 & \text { Automatic enhancement of } \mathfrak{s u}(2) \text { gauge algebra } & 12\end{array}$

$\begin{array}{lll}4.2 .1 & \mathfrak{s u}(2) \rightarrow(\mathrm{SU}(2) \times \mathrm{SU}(2)) / \mathbb{Z}_{2} \text { automatic enhancement } & 12\end{array}$

$\begin{array}{lll}4.2 .2 & \mathfrak{s u}(2) \rightarrow(\mathrm{SU}(2) \times \mathrm{U}(1)) / \mathbb{Z}_{2} \text { automatic enhancement } & 15\end{array}$

$\begin{array}{lll}4.2 .3 & \mathfrak{s u}(2) \rightarrow \mathrm{SO}(3) \text { and massless charge sufficiency } & 16\end{array}$

$4.3 T=0$ models with large $\mathfrak{s u}(N)$ gauge algebras at $b=1 \quad 16$

$\begin{array}{lll}4.4 & \text { Other } \mathrm{SU}(N) \text { examples } & 19\end{array}$

$\begin{array}{lll}\text { 4.4.1 Enhancements of } \mathfrak{s u}(3) \text { and beyond for general } b & 19\end{array}$

4.4.2 Enhancements of $\mathfrak{s u}(N)$ for $g=0$, small $b \cdot b \quad 20$

$\begin{array}{lll}4.5 & \text { Examples with } \mathfrak{s p}(N) \text { and } \mathfrak{s o}(N) \text { algebras } & 21\end{array}$

5 Abelian examples $\quad 22$

$\begin{array}{lll}5.1 & \mathrm{U}(1) \\ 5.2 & \mathrm{U}(1) & 22\end{array}$

$\begin{array}{lll}5.2 \mathrm{U}(1) \text { infinite families } & 23\end{array}$

5.3 Infinite family of $\mathfrak{s u}(13) \oplus \mathfrak{u}(1)$ models 29

5.4 Automatic enhancement for $(\mathrm{SU}(3) \times \mathrm{SU}(2) \times \mathrm{U}(1)) / \mathbb{Z}_{6}$ models 30

6 Positivity conditions and local conditions for automatic enhancement $\quad 30$

$\begin{array}{lll}7 & \text { Exotic matter and discrete gauge groups } & \mathbf{3 3}\end{array}$

7.1 An $\mathfrak{s u}(2)$ model with two half-hypermultiplets of 4 matter 34

$\begin{array}{lll}7.2 & \text { More exotic matter } & 36\end{array}$

$\begin{array}{lll}7.3 & \text { Discrete gauge groups } & 38\end{array}$ 
8 Automatic enhancement in lower-dimensional theories

8.1 Automatic enhancement in $4 \mathrm{D} \mathcal{N}=1$ F-theory models 39

8.2 Automatic enhancement in 5D and 4D theories with 8 supercharges 39

9 Conclusions and further directions $\quad 40$

A Explicit analysis of rational sections $\quad 42$

A.1 $(\mathrm{SU}(2) \times \mathrm{U}(1)) / \mathbb{Z}_{2}$ model $\quad 42$

A.2 $(\mathrm{SU}(2) \times \mathrm{SU}(2)) / \mathbb{Z}_{2}$ and $\mathrm{SU}(2) / \mathbb{Z}_{2}$ models 42

$\begin{array}{lll}\text { B } U(1) \times U(1) \text { model } & 43\end{array}$

C Absence of adjoint matter on $C$ when $C+K_{B}$ is not effective 44

D Another test of automatic enhancement in a U(1) model 47

\section{Introduction}

Six-dimensional supergravity is a promising arena in which to understand the relationship between possible low-energy theories of quantum gravity and UV completions in the context of string theory. In ten dimensions, it has been shown that all supersymmetric theories of gravity coupled to gauge fields in flat space-time that satisfy anomaly cancellation and all other known quantum consistency conditions are realized, at the level of massless spectra, through some version of string theory $[1,2]$. There has been recent progress towards showing that a similar "string universality" principle holds for supergravity theories in eight (as well as nine and seven) space-time dimensions [3, 4]. In six dimensions, the space of theories becomes significantly more rich: six is the largest space-time dimension in which matter fields can arise in representations other than the adjoint for a supersymmetric quantum theory of gauge fields and gravity. The space of $6 \mathrm{D}$ supergravity theories is, however, still reasonably manageable. For the most part, the theory consists of a set of distinct continuous branches connected by various transitions into a single large metamoduli space. The massless spectra in these different branches are strongly constrained by gravitational, gauge-gravitational and pure gauge anomaly conditions.

F-theory [5-7] provides a powerful nonperturbative tool for exploring a large part of the global meta-moduli space of $6 \mathrm{D}$ supergravity theories in terms of the geometric moduli spaces of elliptic Calabi-Yau threefolds, which are linked together in a large connected network [8-11] by transitions that can change the number of tensor multiplets [7, 12], as well as standard Higgs transitions and more exotic "matter transitions" [13]. There is a close connection between the elliptic Calabi-Yau geometry of F-theory and the structure of the string charge lattice and anomaly coefficients in the corresponding low-energy $6 \mathrm{D}$ supergravity theory, making it possible in many branches of the theory to clearly identify the geometric ingredients needed for the UV completion through F-theory of the given 
branch of the low-energy 6D supergravity moduli space (see, e.g., [9]). It was conjectured in [14] that string universality should hold for the massless spectra of $6 \mathrm{D}$ supergravity theories, so that any massless spectrum associated with a supersymmetric theory of gauge fields and gravity not realized in string or F-theory should be provably inconsistent. At this point in time, however, despite ongoing progress both in understanding the range of possible F-theory constructions and in identifying new quantum consistency conditions for $6 \mathrm{D}$ supergravity theories, there are still many theories, including some infinite classes of theories, in the apparent swampland $[15]^{1}$ of $6 \mathrm{D}$ low-energy supergravity theories that obey anomaly cancellation as well as all other known quantum consistency conditions and yet have no known F-theory realization.

In this paper we find a unifying principle that underlies a range of the theories in the current apparent 6D supergravity swampland, and we formulate a precise conjecture for a class of theories that seem to be impossible to realize in F-theory despite satisfying the known 6D quantum consistency conditions. This paper was motivated by the observation that certain apparently consistent $(\mathrm{SU}(3) \times \mathrm{SU}(2) \times \mathrm{U}(1)) / \mathbb{Z}_{6}$ models cannot be realized in F-theory by a universal Weierstrass realization identified earlier by the authors [17]. Instead, the rank of the gauge group is forced to increase when the anomaly coefficients associated with the gauge factors are sufficiently large. This kind of enhancement had also been seen in simple $\mathrm{SU}(2)$ models $[18,19]$. Further exploration has revealed that indeed many models that seem consistent but resist an F-theory realization fit with this same general pattern. In this paper, we generalize these observations in the formulation of a conjecture, and we elaborate on range of different situations where this kind of automatic enhancement occurs. If the general conjecture we present here and its stronger forms for specific gauge groups hold for general $6 \mathrm{D}$ supergravity theories it would rule out many theories in the apparent $6 \mathrm{D}$ supergravity swampland.

The structure of this paper is as follows: In section 2, we formulate the central conjecture, which essentially states that given any $6 \mathrm{D}$ supergravity theory that has a gauge group and matter content contained within those of another consistent theory that can be realized in F-theory, but where the former theory cannot be reached by a Higgs deformation of the latter theory, the former theory does not arise in F-theory. In section 3, we give a brief review of some of the main types of theories that appear currently to be in the $6 \mathrm{D}$ supergravity swampland. In the following two sections, we go through a number of examples where we can demonstrate explicitly that the conjecture holds for standard F-theory constructions. section 4 addresses a variety of examples with nonabelian gauge theories, including non-Higgsable clusters and theories with gauge group $\mathrm{SU}(N)$, and section 5 describes examples with an abelian gauge group, including in particular some infinite families of apparently consistent theories with gauge group U(1) and arbitrarily large charges that

\footnotetext{
${ }^{1}$ Note: the term "swampland" is used slightly differently by different authors. Here, we refer to the "apparent swampland" as those theories that satisfy all known quantum consistency conditions for theories coupled to quantum gravity but have no known string realization; a theory can be removed from the apparent swampland if a string realization is found, or if a new quantum consistency constraint is identified that rules out the theory. A recent review of work on swampland related problems, mostly focusing on questions separate from the 6D supergravity issues addressed here, can be found in [16].
} 
were previously known. In section 6, we give a slightly stronger characterization of the conjecture in terms of positivity/effectiveness for a variety of the specific simple cases studied, and also give a sufficient local condition in some cases for a theory to either be enhanced or broken in the F-theory realization. In section 7, we consider some more complicated cases, focusing in particular on theories with exotic nonabelian matter representations and those with discrete gauge groups. The discussion includes situations in which the application of the conjecture formulated here is less clear but there are interesting related questions. In section 8 , we briefly consider how the ideas of this paper may be extrapolated to lowerdimensional supergravity theories such as $\mathcal{N}=1$ and $\mathcal{N}=24 \mathrm{D}$ supergravity theories, and in section 9 we make some concluding remarks.

The work in this paper is complemented by a separate but related work also nearing completion [19] that addresses questions of completeness of the charge spectra and massless charge spectra in $6 \mathrm{D}$ supergravity theories and their F-theory realizations.

\section{Statement of the conjecture}

The basic proposal of this paper is the following conjecture for 6D F-theory and supergravity theories:

Automatic Enhancement Conjecture. Given any anomaly-consistent $6 D$ supergravity theory with gauge group $G$ and massless matter hypermultiplets in a set of representations $M$, such that there exists another anomaly-consistent theory with gauge group $G^{\prime} \supset G$ and matter representations $M^{\prime} \supseteq M$ that is realized in F-theory but cannot be broken through a supersymmetry-preserving Higgsing process to the theory with gauge group $G$, the theory with gauge group $G$ cannot be realized in F-theory. Equivalently, trying to tune the gauge group and matter content $G, M$ in an F-theory Weierstrass model leads to an automatic enhancement of the singularity types to a theory with gauge group and matter content $G^{\prime} \supset G, M^{\prime} \supseteq M$.

Some comments on this conjecture:

- Note that the theory with gauge group and matter content $G^{\prime}, M^{\prime}$ must contain the theory with $G, M$ in the strong sense that all other features of the theory must be the same. In particular, the string charge lattice that contains the anomaly coefficients (associated with $H_{1,1}(B, \mathbb{Z})$ of the compactification base $B$ in an F-theory description; see, e.g., [9]) and the positivity cone on this lattice (associated with the cone of effective divisors in F-theory) must be the same, and the anomaly coefficients of the gauge factors in $G$ must match their counterparts in $G^{\prime}$.

- We have stated this conjecture in the context of F-theory. Much of the bulk of this paper consists of a variety of examples where this conjecture holds for standard tunings in the F-theory context. While in some cases, such as the situation of nonHiggsable clusters (section 4.1), the conjecture can be explicitly proven, in other situations the evidence is only partial even in the F-theory context. Nonetheless, it is tempting to speculate that this conjecture holds not only in F-theory but for general 
6D supergravity theories. If so, this would eliminate many of the known examples of $6 \mathrm{D}$ supergravity theories in the swampland of theories that appear consistent by all known criteria but which are not realized in F-theory.

- This conjecture complements the Massless Charge Sufficiency Conjecture made in [19], which asserts that the massless charge lattice of a $6 \mathrm{D}$ supergravity theory generates the full charge lattice of the theory. As discussed further in section 4.2.3, in some cases such as the enhancement of a theory with gauge algebra $\mathfrak{s u}(2)$ with a large anomaly coefficient, the massless charge sufficiency conjecture is closely related to the automatic enhancement conjecture and can be similarly interpreted as leading to the addition of extra elements in the coroot lattice of the gauge group $G$.

- On a practical level, enhancements typically occur because the choice of divisor classes necessary to obtain the correct matter spectrum causes the elliptic fibration to take some special structure. A parameter could be forced to be zero because its divisor class is ineffective, or multiple parameters may share common factors because their classes only admit reducible sections. And if a parameter's divisor class is trivial, the elliptic fibration may exhibit enhanced features such as the presence of additional rational sections. Since these effects occur in the geometry, one may naively wonder if the $G, M$ model could be obtained from the $G^{\prime}, M^{\prime}$ model by a T-brane deformation $[20,21]$. However, examples of the Automatic Enhancement Conjecture exhibit a field-theoretic obstruction to Higgsing $G^{\prime}$ to $G$. T-brane deformations should respect these obstructions, implying that automatic enhancement cannot be evaded in this fashion.

- The conjecture as stated above corresponds to the complete $6 \mathrm{D}$ supergravity theory and its F-theory realizations, and is stated in a very general way. Most of the examples we describe here also admit a more specific and stronger formulation in a way that depends on the particular group/algebra in question. In particular, in many cases the enhancement mechanism is required when the anomaly coefficients of the relevant gauge factors theory violate a particular positivity condition, associated with an effectiveness condition for the corresponding F-theory curves. When this condition is violated either an enhancement must occur or there cannot exist a compatible Ftheory model. In many cases these positivity constraints lead to sufficient conditions that can be formulated completely locally in the F-theory geometry. We illustrate these positivity and local aspects in many of the examples described in the following sections, and summarize these versions of the conjecture in section 6 .

\section{Review of $6 \mathrm{D}$ supergravity, F-theory realizations, and the swampland}

In this section we briefly review some aspects of the current apparent "swampland" of $6 \mathrm{D}$ supergravity theories that appear consistent from all known quantum consistency conditions but that have no known F-theory realization. 


\subsection{D supergravity theories and their realizations in F-theory}

A $6 \mathrm{D} \mathcal{N}=1$ supergravity theory is characterized by a massless spectrum that contains a fixed number of tensor multiplets $T$, a gauge group $G$, and a set of hypermultiplet matter fields $M$ living in certain representations of the gauge group $G$. The gauge group can have nonabelian and abelian factors, as well as discrete components. The conditions of gravitational, gauge-gravitational, and pure gauge anomaly cancellation [22, 23] impose fairly strong constraints on the set of possible gauge groups and massless matter spectra. When the number of tensor multiplets satisfies $T<9$, it has been proven that there are a finite number of distinct possible massless spectra $G, M$ for theories with purely nonabelian gauge groups $G$ [9]. On the other hand, even when there are no tensor multiplets $(T=0)$, there exist infinite families of theories with a simple $\mathrm{U}(1)$ gauge group and different combinations of charged matter fields $[24,25]$ that satisfy anomaly cancellation and other known consistency conditions.

A wide range of $6 \mathrm{D} \mathcal{N}=1$ supergravity theories can be realized using F-theory [5-7]. A six-dimensional F-theory model is defined by an elliptic Calabi-Yau threefold $X$, and can be thought of as a nonperturbative type IIB compactification on the (real four-dimensional) complex Kähler surface $B$ that acts as the base of the elliptic fibration $X$. Introductions to the relevant aspects of F-theory compactifications can be found in $[10,26]$. The set of $6 \mathrm{D}$ F-theory models forms a connected meta-moduli space; the allowed bases are connected through nonperturbative transitions that change the number of tensor multiplets $[7,12]$, and over any given base $B$ the set of valid $\mathrm{F}$-theory models forms a continuous connected moduli space parameterized by the coefficients $f, g$ in the Weierstrass model

$$
y^{2}=x^{3}+f x+g .
$$

Here $f, g$ can be thought of as functions on the base $B$, or more precisely as sections of line bundles $\mathcal{O}\left(-4 K_{B}\right), \mathcal{O}\left(-6 K_{B}\right)$, where $K_{B}$ is the canonical class of the base. Codimensionone loci where the elliptic fiber becomes singular lead to nonabelian factors of the gauge group $G$, while abelian and discrete factors have a more subtle global characterization. Codimension-two loci where the elliptic fiber becomes singular in general characterize massless matter fields in the 6D supergravity theory. There is thus a close correspondence between the geometry of the F-theory compactification and the structure of the low-energy $6 \mathrm{D}$ supergravity theory.

One particularly important connection between low-energy physics and F-theory is that there is a simple geometric interpretation of the anomaly coefficients $a, b_{i}, \tilde{b}_{\alpha}$ associated with terms in the $6 \mathrm{D}$ supergravity action of the form $B \wedge R \wedge R, B \wedge F_{i} \wedge F_{i}, B \wedge F_{\alpha} \wedge F_{\alpha}$ respectively, with $F_{i}, F_{\alpha}$ denoting nonabelian and abelian gauge field strength factors. In particular, $a$ corresponds to the canonical class $K_{B}$, and $b_{i}$ corresponds to the locus $C_{i}$ in $B$ where the nonabelian gauge factor $G_{i}$ is supported. Note that for $k$ abelian factors there are anomaly coefficients $\tilde{b}_{\alpha \beta}$ associated with terms $B \wedge F_{\alpha} \wedge F_{\beta}$; we use the notation $\tilde{b}_{\alpha}=\tilde{b}_{\alpha \alpha}$. These are special cases of the more general identification between the string charge lattice $\Lambda$ of the $6 \mathrm{D}$ supergravity theory, in which $a, b_{i}, \tilde{b}_{\alpha}$ are elements, and the lattice $H_{1,1}(B, \mathbb{Z})$ given by the set of algebraic curves in the base $B$, including $-K_{B}$ and $C_{i}$, with the natural 
intersection form on homology. Because of this isomorphism, we will frequently move freely back and forth between $a, b_{i} \in \Lambda$ and $K_{B}, C_{i} \in H_{1,1}(B, \mathbb{Z})$ in various parts of the discussion. A useful feature of this correspondence is that the arithmetic genus of a (possibly singular) curve $C_{i}$ supporting a nonabelian gauge factor $G_{i}$ is encoded in the anomaly coefficients of the low-energy theory through $[27,28]$

$$
g_{i}=\frac{1}{2}\left(a \cdot b_{i}+b_{i} \cdot b_{i}\right)+1 .
$$

To organize our brief review of some classes of models in the $6 \mathrm{D}$ supergravity swampland, we will find it helpful to distinguish "generic" from exotic matter fields for a given gauge group. A precise definition of which matter fields are generic for a $6 \mathrm{D}$ supergravity theory with a fixed gauge group $G$ (and for fixed anomaly coefficients, which are assumed to be not too large) can be given by identifying the generic matter fields as those that arise on the branch of moduli space of greatest dimension for fixed $G$ and associated anomaly coefficients [29]. This definition of generic turns out to match with the simplest types of codimension-two singularities that give matter in the F-theory picture and also ties in naturally to the structure of the anomaly constraints: at least for simple theories, the number of generic matter representations is equal to the number of anomaly constraints, so in these cases there is a unique generic matter content for fixed $G$ once the anomaly coefficients are fixed.

Another aspect of F-theory constructions that is relevant for the analysis of this paper is that for a given gauge algebra $\mathfrak{g}$, the moduli space of models with matter fields in the generic representations is captured in many cases by a "universal" 2 Weierstrass model, with a general algebraic form parameterized by the classes of the curves supporting the gauge factors. In [17] we developed a systematic Jacobian-based methodology for checking that a given family of Weierstrass model constructions is universal by checking that the number of independent free complex parameters in the Weierstrass model matches the dimensionality of the branch of the moduli space determined by the number of uncharged scalars. Such universal Weierstrass models include, for example, the well-known Tate tunings of most nonabelian gauge factors [30], the Morrison-Park U(1) Weierstrass models [18], and the universal $(\mathrm{SU}(3) \times \mathrm{SU}(2) \times \mathrm{U}(1)) / \mathbb{Z}_{6}$ models developed in [17]. Note that universal Weierstrass models are also known for some classes of models containing specific combinations of representations that include exotic matter.

\subsection{The $6 \mathrm{D}$ supergravity swampland}

While for many classes of theories there is a fairly close match between the set of possible Ftheory constructions and the $6 \mathrm{D} \mathcal{N}=1$ supergravity theories that are allowed from anomaly cancellation and other known quantum consistency conditions, there are also many theories that appear to be in the swampland. In [9, 24, 25], for example, some infinite families of apparent swampland models were identified. In general, both supergravity and F-theory models are understood best for theories with no tensor multiplets $(T=0)$, and with

\footnotetext{
${ }^{2}$ In [17], these universal Weierstrass model constructions were referred to as "generic"; here we change terminology to "universal" to avoid confusion with other uses of the term generic.
} 
nonabelian gauge groups and generic matter representations. The structure of the theories becomes more complicated as the number of tensor multiplets increases, particularly at $T \geq 9$, when abelian and discrete gauge factors are included, and when exotic (non-generic) matter representations are considered. Over the last decade or so, there has been gradual progress in better understanding both F-theory constructions of these more complicated classes of models and also in identifying further quantum consistency constraints that must be satisfied by any consistent $\mathcal{N}=16 \mathrm{D}$ supergravity theory; as these theories are better understood, further classes of apparent swampland models have also been identified. We briefly review some of this work here, first summarizing some additional constraints on $6 \mathrm{D}$ supergravity and F-theory constructions, and then focusing on some of the simplest and largest classes of apparent swampland models, for which the Automatic Enhancement Conjecture is relevant.

\subsubsection{Constraints on 6D supergravity theories}

In addition to the constraints of local and global anomaly cancellation, a consistent $6 \mathrm{D}$ $\mathcal{N}=1$ supergravity theory must satisfy a variety of further quantum consistency conditions. We briefly summarize some of these further constraints here.

One simple constraint is that the gauge kinetic term proportional to $F^{2}$ must have a negative coefficient; otherwise the theory has a perturbative instability. This leads through supersymmetry to the condition that the anomaly coefficients $b_{i}, \tilde{b}_{\alpha}$ must lie in the positive cone of the $6 \mathrm{D}$ string charge lattice $\Lambda$. This was used, for example, in [31] to rule out an infinite family of models that had appeared to lie in the swampland [32].

Another constraint, proven in [33], is that the charge lattice $\Lambda$ must be unimodular (self-dual) under the Dirac pairing between strings; this follows automatically from Ftheory, where the lattice $H_{1,1}(B, \mathbb{Z})$ is always unimodular, but this condition must also hold more generally in $6 \mathrm{D}$ supergravity, ruling out other classes of potential swampland models.

Some further constraints on the anomaly coefficients $a, b_{i}, \tilde{b}_{\alpha}$ were identified in [34, $35]$ using global and local anomaly cancellation conditions (a subset of these constraints were also found in $[25,36]$ ). These constraints are stronger if one makes the additional assumption that the $6 \mathrm{D}$ theory can be defined on any spin manifold with an arbitrary gauge bundle. For example, under these assumptions the anomaly coefficient $a$ must be a characteristic vector in the subspace of $\Lambda$ spanned by the nonabelian anomaly coefficients $b_{i}$, meaning that $a \cdot b_{i}+b_{i} \cdot b_{i} \in 2 \mathbb{Z}$. This is true for all F-theory compactifications (since, e.g., the genus (3.2) is always an integer), but can rule out some theories that might appear to be in the swampland.

There are a number of further constraints that are known to hold in F-theory but have not been proven as consistency conditions for general quantum $6 \mathrm{D}$ supergravity theories. Theories satisfying the known 6D supergravity quantum consistency conditions but violating these F-theory constraints appear to be in the swampland, pending a proof that these conditions are more generally necessary for $6 \mathrm{D}$ supergravity consistency. The primary goal of this paper is to present evidence that the automatic enhancement conjecture should be a similar F-theory constraint. We summarize briefly here some other constraints in this class. Note that beyond standard F-theory models there are also other possible con- 
structions such as the "frozen" phase of F-theory [37], which can realize some models that otherwise may appear to be in the swampland. As far as we know the models we study here do not have any such realization through any formulation of string theory including the standard class of F-theory constructions.

One constraint that follows directly from F-theory is the Kodaira constraint [9], which states that $-12 a-\sum_{i} \nu_{i} b_{i}$ must lie in the positivity cone of $\Lambda$, which corresponds to the cone of effective divisors in $H_{1,1}(B, \mathbb{Z})$. Here $\nu_{i}$ corresponds to the number of 7-branes needed to form the nonabelian factor $G_{i}$ of the gauge group, e.g., for $\mathrm{SU}(N), \nu=N$. Various $6 \mathrm{D}$ supergravity theories that look otherwise consistent violate this condition and hence cannot be realized in F-theory; we mention some explicit examples later in this section.

Another constraint that is widely expected to hold in all consistent quantum theories of gravity in dimensions $D>3$ is the condition of charge completeness [38], which states that there exist excitations of the theory with all possible charges in the weight lattice of the symmetry group $G$. This was proven recently for all quantum gravity theories with a holographic dual [39]. This condition is proven for all 6D F-theory models in [19] using Poincaré duality, although there is no general proof for flat space $6 \mathrm{D}$ supergravity theories.

A further class of constraints can be identified if one assumes that the charge completeness hypothesis holds in the sense that there are dynamical string excitations for every charge in the string lattice $\Lambda$. Under this assumption, there must be a consistent anomalyfree theory on the worldvolume of such strings, which leads to additional constraints on the set of allowed spectra for $6 \mathrm{D}$ supergravity theories. This method was used in [40], for example, to show that the rank of the gauge group of 10D type I string theory must match with the known value of $\mathfrak{s o}(32)$. In [2], this approach was used to show that consistency of the theory on the string worldvolume places additional constraints on the spectra of $6 \mathrm{D}$ supergravity theories; among other things, these constraints would rule out several infinite families of apparent swampland models with $T>9$ that were found in [9]. In [41], this approach was used to place bounds on the number of abelian gauge factors that can arise in a $6 \mathrm{D}$ supergravity theory, improving on previous bounds found from anomaly conditions in $[24]$.

The focus in this paper is on 6D supergravity models that obey all of the abovementioned constraints but still have no F-theory realization. To organize the discussion we summarize some of the models that are still in the apparent swampland, including some that violate the conditions above that are known to hold in F-theory vacua but are otherwise apparently consistent $6 \mathrm{D}$ supergravity theories.

\subsubsection{Apparent swampland for $T=0$, nonabelian $G$, and generic matter}

We begin with the simplest set of cases, with no tensors, a nonabelian gauge group, and generic matter. Because of the absence of tensor fields, the anomaly coefficients $a, b_{i}$ are integers. We focus first on theories with gauge group $\mathrm{SU}(N)$ and then consider other gauge groups. For $G=\mathrm{SU}(N)$, generic matter fields are the fundamental and adjoint representation, as well as the two-index antisymmetric representation when $N \geq 4$ [29].

With gauge group $\mathrm{SU}(2)$, there are anomaly-allowed theories for anomaly coefficients $1 \leq b \leq 12$ [29]. Naively, it seems that these theories can all be constructed directly in 
F-theory by directly tuning the appropriate gauge factor in the Weierstrass model. When $b=9,10,11,12$, however, complications arise. In the case $b=12$, as discussed further in [19], this construction gives a theory with $\mathbb{Z}_{2}$ Mordell-Weil torsion, so the gauge group is actually $\mathrm{SO}(3)$; in this case, the massless charge sufficiency conjecture stated in that paper asserts that the pure $\mathrm{SU}(2), b=12$ theory cannot be realized in F-theory. For $b=10,11$, it was observed in [19] that the naive tuning of this model gives an extra $\mathrm{SU}(2)$ factor and Mordell-Weil torsion so $G=(\mathrm{SU}(2) \times \mathrm{SU}(2)) / \mathbb{Z}_{2}$. These cases, as well as the case $b=9$, are in the apparent swampland and satisfy the conditions of the Automatic Enhancement Conjecture, and are described in more detail in section 4.2.1 and section 4.2.2.

For gauge group $\mathrm{SU}(N)$, there are anomaly-allowed theories for $1 \leq b \leq 24 / N$. Again, naively these can all be constructed using a Tate tuning [30]. But again, in some cases the resulting model does not have the desired $\mathrm{SU}(N)$ gauge group. It was pointed out in $[42,43]$ that for $b=1$ this construction does not give theories with gauge groups $\mathrm{SU}(21)$ and $\mathrm{SU}(23)$ and generic matter, so these are in the apparent swampland. These cases, as well as the other cases with $N \geq 18$ are examples of the Automatic Enhancement Conjecture, as we describe in more detail in section 4.3. Note that the case of SU(24), which lacks fundamental matter and has an actual gauge group $\mathrm{SU}(24) / \mathbb{Z}_{2}$ is an example where Mordell-Weil torsion is forced, in accord with the Massless Charge Sufficiency Conjecture [19].

Similar apparent swampland theories arise for $\mathfrak{s p}(N)$ and $\mathfrak{s o}(N)$; we discuss some cases in section 4.5 .

\subsubsection{Apparent swampland with abelian $G$}

When we include abelian $\mathrm{U}(1)$ gauge factors, the story becomes more complicated. In F-theory, U(1) factors come from global features of the geometry, which makes them more subtle to understand. For theories with a gauge group U(1) and generic $(q=1,2)$ matter, there are apparent swampland models closely related to the simplest $\mathrm{SU}(2)$ models discussed above; we describe in section 5.1 how these models also satisfy the Automatic Enhancement Conjecture. Even for theories with a single $\mathrm{U}(1)$ as the gauge group and $T=0$, when larger charges are considered there are infinite families of charge configurations that satisfy anomaly cancellation conditions [24, 25], even though there can only be a finite number of distinct spectra compatible with F-theory constructions [9]. In section 5.2, we show that at least the simplest of these families satisfies the conditions of the Automatic Enhancement Conjecture.

Combining nonabelian and abelian factors, one can also find infinite families of anomaly-free theories with gauge algebras of the form $\mathfrak{s u}(N) \oplus \mathfrak{u}(1)$, for example. (Some infinite families of this type with $T=1$ were constructed in [24], and similar constructions are possible with $T=0$.) In section 5 , we show that the previously identified infinite classes of theories of this kind satisfy the conditions of the stronger version of the Automatic Enhancement Conjecture that would render these theories inconsistent, although we also identify some further infinite families for which the application of the conjecture is less transparent. We also show in this section that the Automatic Enhancement Conjecture applies to some theories with gauge group $(\mathrm{SU}(3) \times \mathrm{SU}(2) \times \mathrm{U}(1)) / \mathbb{Z}_{6}$ when constructed with the universal Weierstrass form identified in [17]. 
Going beyond single $\mathrm{U}(1)$ factors, at this point our understanding of F-theory models with multiple $\mathrm{U}(1)$ factors and/or discrete gauge group factors is still sufficiently limited that it is difficult to clearly delineate the swampland. This is in large part because abelian $\mathrm{U}(1)$ and discrete factors come from global aspects of the F-theory geometry, unlike nonabelian gauge factors that come from local structure. We do not attempt to give any systematic analysis here of theories with multiple U(1) factors or discrete gauge factors, though we make some general comments about theories with discrete gauge symmetries in section 7.3 .

\subsubsection{Apparent swampland with $T>0$}

When the number of tensor multiplets is nonzero, $T>0$, the structure of the theory becomes more complicated, and questions about the swampland have additional complications. The anomaly coefficients $a, b_{i}, \tilde{b}_{\alpha}$ become vectors as $\Lambda$ becomes a signature $(1, T)$ lattice, corresponding to $H_{1,1}(B, \mathbb{Z})$. There is a positivity cone for strings in $\Lambda$, which in the F-theory description corresponds to effective divisors in $B$ but which is not as well understood in the $6 \mathrm{D}$ supergravity context. A variety of apparent swampland models may arise corresponding to lattices $\Lambda$ with positivity cones that do not match any F-theory geometry. The condition identified in [34] that $a$ must be a characteristic vector, at least for the nonabelian anomaly coefficients, puts some concrete constraint on the allowed combinations of $\Lambda, a$, but there are still many theories at larger $T$ that appear consistent and yet have no F-theory realization. We focus here on some of these that are most relevant for this story.

The most basic new feature that can arise at $T>0$ is the appearance of a vector $x \in \Lambda$ that lies in the positivity cone and satisfies $x \cdot x<0$. When an F-theory construction involves a base $B$ that contains an effective curve $C$ with $C \cdot C<-2$, the negative normal bundle on $C$ forces a Kodaira singularity in the elliptic fibers over that curve that gives a gauge group of at least $\mathrm{SU}(3)$ in the corresponding 6D supergravity theory [44]. From the point of view of supergravity, there is no obvious inconsistency with an element $x$ satisfying $x \cdot x<-2$ lying in the positivity cone without a corresponding gauge factor in the theory. Thus, such models lie in the apparent swampland. In section 4.1, we show that these models are a simple example of the Automatic Enhancement Conjecture. Note that under the assumption of charge completeness for the string charge lattice, one could alternatively argue that anomaly cancellation on the worldvolume of a string with string charge $x$ can only be satisfied in the presence of the appropriate non-Higgsable gauge factor $G \supset \mathrm{SU}(3)$, using analysis along the lines of [2, 45-47].

Already with a single tensor multiplet $(T=1)$, the range of possible theories expands significantly and there are more theories that have been identified in the apparent swampland. In particular, in [24] there are infinite families of apparent swampland models identified with $T=1$ and gauge algebras $\mathfrak{u}(1)$ and $\mathfrak{s u}(13) \oplus \mathfrak{u}(1)$. Both of these families can be ruled out by the Automatic Enhancement Conjecture, as we discuss in section 5. The $\mathrm{U}(1)$ family is closely related to the infinite family of $\mathrm{U}(1)$ theories at $T=0$ found in [25], but the $\mathfrak{s u}(13) \oplus \mathfrak{u}(1)$ family is somewhat more subtle. 
When $T \geq 9$, the classification of theories gets even more complicated, due in particular to the fact that $a \cdot a=9-T$ ceases to be positive (this condition follows from gravitational anomaly cancellation). An illustration of some of the complications that arise at $T \geq 9$ is given in appendix C. For $T \geq 9$, there is no proof known that even constrains the number of distinct spectra for nonabelian gauge groups and matter to be finite, though as mentioned earlier some of the infinite families of apparent swampland models identified in [9] violate the conditions found in [2] that hold when string charge completeness is assumed.

\subsubsection{Apparent swampland with exotic matter for nonabelian $G$}

In addition to the generic matter representations for a nonabelian gauge group $G$ there are also non-generic matter representations possible that can still satisfy anomaly cancellation. Three-index antisymmetric representations of $\mathrm{SU}(N)$ for $N=6,7,8$ are fairly well understood $[13,30,42,48]$ and can arise through a Tate-type tuning of the Weierstrass model [49]. Related exotic multi-charged matter representations include, e.g., the $(\mathbf{6}, \mathbf{2})$ of $\mathrm{SU}(4) \times \mathrm{SU}(2)$ and the trifundamental of $\mathrm{SU}(2)$. More exotic matter representations can be characterized as having a non-vanishing "genus" contribution (3.2), corresponding to the arithmetic genus of a singularity in the divisor supporting the gauge factor in an F-theory realization. In [28], it was argued that only a fairly limited set of exotic matter representations for nonabelian groups can be realized in F-theory without having a codimension two locus where $f, g$ vanish to degrees $(4,6)$, which generally signifies a superconformal sector [50-52]. These limited higher-genus exotic matter representations that can be realized through F-theory models on a singular gauge divisor basically contain only the two-index symmetric representation of $\mathrm{SU}(N)$, the three-index symmetric representation of $\mathrm{SU}(2)$, and analogous representations of $\operatorname{Sp}(N)$. It has been suggested that some other exotic representations such as the $(\mathbf{5 6}, \mathbf{2})$ of $\mathrm{E}_{7} \times \mathrm{SU}(2)$, which naively involves a $(4,6)$ locus, may be possible without a superconformal sector [53] through T-brane magic. Though this is not well understood in F-theory, it is believed that there are heterotic models containing this matter representation without superconformal sectors.

With this general classification of exotic matter, there are two kinds of apparent swampland theories. First, there are anomaly-consistent theories containing the exotic matter representations that can arise from F-theory constructions on singular divisors, but in configurations that do not seem to arise from F-theory. Some examples of such apparent swampland theories were described in [28]; we show in section 7.1 that some such examples satisfy the conditions of the Automatic Enhancement Conjecture. Second, are any theories that contain matter in representations of nonabelian gauge factors not believed to arise in consistent F-theory models. We make some brief comments on such models in section 7.2.

\section{Nonabelian examples}

\subsection{Non-Higgsable clusters}

One of the simplest situations in which automatic enhancement must occur in F-theory is when $G$ is trivial and there is a primitive positive charge $q$ in the string charge lattice 
with Dirac pairing $q \cdot q<-2$ with itself and $q \cdot a=-q \cdot q-2$ with the $B R R$ anomaly coefficient $a$. In any F-theory construction where the complex surface base $B$ on which the theory is compactified contains a curve $C$ of self-intersection -3 or below, everywhere in the F-theory moduli space of elliptically fibered Calabi-Yau threefolds parameterized by Weierstrass models over $B$ there is a singularity in the elliptic fibration that forces at least a gauge algebra $\mathfrak{s u}(3)$ with an anomaly coefficient $b=C$, where the string charge lattice containing the anomaly coefficient is identified with $H_{1,1}(B, \mathbb{Z})$ in any $6 \mathrm{D}$ F-theory model [44]. Thus, in such a theory with a primitive positive charge $b$ where $b \cdot b=-3$, $a \cdot b=1$ there is an automatic enhancement $G=1 \rightarrow G^{\prime} \supseteq \mathrm{SU}(3)$. the self-intersection $b \cdot b$ decreases, the rank of the forced gauge group increases, with, e.g., $b \cdot b=-4 \Rightarrow G^{\prime} \supseteq \mathrm{SO}(8)$, $\ldots, b \cdot b<-8 \Rightarrow G^{\prime}=\mathrm{E}_{8}$, and with $b \cdot b \leq-13$ not allowed in any F-theory model. In all these situations, the gauge group $G^{\prime}$ is "non-Higgsable" in the sense that there is no local Higgsing deformation that can break the gauge group while preserving supersymmetry. In particular, in these cases there is no Higgsing from $G^{\prime} \rightarrow G$, as expected in the Automatic Enhancement Conjecture.

Note that in this case the Automatic Enhancement Conjecture can be stated purely locally: we expect that any charge $b$ in the positivity cone of the string charge lattice $\Lambda$ that satisfies $b \cdot b<-2$ and satisfies $a \cdot b=-2-b \cdot b$ (corresponding to $g=0$ from eq. (3.2)) must support a gauge factor $G^{\prime} \supseteq \mathrm{SU}(3)$, with correspondingly larger factors for more negative $b \cdot b$. Furthermore, for similar reasons there cannot be any a curve in the positivity cone with $g>0$ and $b \cdot b<0$ in any F-theory construction [44].

In this class of automatic enhancements there is a complete proof that the enhancement must occur in the context of F-theory. In other cases, our understanding of the F-theory geometry is less complete and while we can give many examples of standard tunings that lead to automatic enhancement, the proof that this must always occur even in the Ftheory context is less complete. Whether the automatic enhancement associated with non-Higgsable clusters can be proven purely in the $6 \mathrm{D}$ supergravity context through other approaches is also discussed briefly in section 3.2.4.

\subsection{Automatic enhancement of $\mathfrak{s u}(2)$ gauge algebra}

Another very simple set of examples of the kind of automatic enhancement implied by the Automatic Enhancement Conjecture arises in the case of 6D F-theory models with gauge group $\mathrm{SU}(2)$, generic matter (fundamental and adjoint representations), and no tensor multiplets (associated with the base $\mathbb{P}^{2}$ ), where the anomaly coefficient for the gauge group is taken to be $b=10,11$. This was one of the first examples of the automatic enhancement mechanism observed, and was encountered in $[18,19]$. We describe how the automatic enhancement occurs in F-theory for this kind of theory and then describe some further generalizations thereof.

\subsection{1 $\mathfrak{s u}(2) \rightarrow(\mathrm{SU}(2) \times \mathrm{SU}(2)) / \mathbb{Z}_{2}$ automatic enhancement}

A universal form for the Weierstrass model giving a gauge algebra $\mathfrak{s u}(2)$ and the generic fundamental and adjoint matter representations can be constructed either from the Tate form $[30,54]$, or by direct analysis of the general Weierstrass model with the appropriate 
Kodaira singularity type $[42] .{ }^{3}$ Such a universal Weierstrass model with gauge algebra $\mathfrak{s u}(2)$ realized on a curve $\{\sigma=0\}$ takes the form

$$
\begin{aligned}
& f=-\frac{1}{48} \phi^{2}+\sigma f_{1}, \\
& g=\frac{1}{864} \phi^{3}-\frac{1}{12} \phi \sigma f_{1}+\sigma^{2}\left(g_{2}\right) .
\end{aligned}
$$

The classes of $f, g, \sigma$ in the F-theory base are $[f]=-4 K_{B},[g]=-6 K_{B},[\sigma]=C$, where $K_{B}$ is the canonical class of the base. In the $6 \mathrm{D}$ supergravity theory, $K_{B}$ and $C$ correspond to the anomaly coefficients $a, b$ of the $B R^{2}, B F^{2}$ terms respectively; we freely go back and forth between the geometric (curve in $H_{1,1}(B, \mathbb{Z})$ ) and field theory (anomaly coefficient in the $6 \mathrm{D}$ supergravity string charge lattice) notations $K_{B} \leftrightarrow a, C \leftrightarrow b$ throughout the discussion in this and subsequent sections. The discriminant locus associated with the Weierstrass model (4.1) is

$$
\Delta=\frac{1}{16} \phi^{2}\left(\phi g_{2}-f_{1}^{2}\right) \sigma^{2}+\mathcal{O}\left(\sigma^{3}\right) .
$$

The class of the coefficient $g_{2}$ is $\left[g_{2}\right]=-6 a-2 b$. When $b$ is sufficiently large, this class becomes ineffective and $g_{2}=0$. In such a situation, the discriminant becomes $\Delta=\sigma^{2} f_{1}^{2}\left(-\phi^{2}+64 f_{1} \sigma\right) / 16$. This discriminant is divisible by $f_{1}^{2}$, and so there is an automatic enhancement of at least an extra $\mathfrak{s u}(2)$ algebra on the vanishing locus of $f_{1}$, where $\left[f_{1}\right]=-4 a-b$. As shown in [19], the resulting Weierstrass model also has $\mathbb{Z}_{2}$ torsion, so the gauge group becomes $G^{\prime}=(\mathrm{SU}(2) \times \mathrm{SU}(2)) / \mathbb{Z}_{2}$. The fact that the enhanced gauge group arises automatically from the Weierstrass tuning (which is the universal and minimal (UFD) tuning needed for the $\mathfrak{s u}(2)$ algebra) implies that there is no Higgsing from the $G^{\prime}$ theory to the anomaly-free theory with algebra $\mathfrak{s u}(2)$. This corresponds to the absence of adjoint matter fields under the extra $\mathfrak{s u}(2)$ algebra factor on $\left\{f_{1}=0\right\}$ (for further explanation/discussion on this point, see appendix C).

Simple examples The simplest cases in which this enhancement occurs is when the base is $\mathbb{P}^{2}$ and the anomaly coefficient is $b=10$ or $b=11$. In these cases an additional $\mathfrak{s u}(2)$ factor arises on $f_{1}$ with $\left[f_{1}\right]=2,1$ respectively. Since degree one and two curves on $\mathbb{P}^{2}$ are rational curves of genus 0 , the additional factor carries no adjoint matter. Furthermore, the only fundamental matter fields for the additional $\mathfrak{s u}(2)$ are also charged under the original $\mathfrak{s u}(2),{ }^{4}$ so the enhanced $G^{\prime}=(\mathrm{SU}(2) \times \mathrm{SU}(2)) / \mathbb{Z}_{2}$ cannot be Higgsed to the anomaly-free $\mathrm{SU}(2)$ theory on $b=10,11$, matching the conditions of the Automatic Enhancement Conjecture. Note that there are Higgsings of the $G^{\prime}$ theory down to a theory with gauge algebra

\footnotetext{
${ }^{3}$ We assume here that the $\mathfrak{s u}(2)$ algebra is realized through a UFD-type construction. Non-UFD constructions typically give exotic matter representations [28]. Although in principle it may be possible that in some special cases non-UFD models can be realized with only generic matter types, the analysis of appendix D suggests that this will not occur in practice.

${ }^{4}$ This can be seen from the form of the discriminant locus; the only loci over $\left\{f_{1}=0\right\}$ where the vanishing order of $\Delta$ increases are those where either $\sigma=0$, corresponding to matter jointly charged under both $\mathfrak{s u}(2)$ factors, and those where $\phi=0$, corresponding to an enhancement to a Kodaira type III singularity that carries no matter.
} 
$\mathfrak{s u}(2)$; in particular, Higgsing on the bifundamental fields can break these $G^{\prime}$ theories down to an $\mathrm{SO}(3)$ on $b=12$ with only adjoint matter essentially by deforming $f_{1} \sigma \rightarrow \sigma^{\prime}$ where $\sigma^{\prime}$ is a generic degree 12 curve in $\mathbb{P}^{2}$. Such a Higgsing, however, cannot reproduce the original $b=10,11$ theory that acquired the automatic enhancement.

This kind of $\mathfrak{s u}(2)$ enhancement can also occur on many other F-theory bases, not just on $\mathbb{P}^{2}$. On the Hirzebruch surface $\mathbb{F}_{m}$, for example, if $\{\sigma=0\}$ is $n$ times the curve $\tilde{S}$ of positive self-intersection $+m$, then in a basis where $S$ is the curve of self-intersection $-m$ and $F$ is the fiber curve with $F \cdot F=0, F \cdot S=1$ (such that $\tilde{S}=S+m F$ ), the class of $g_{2}$ is

$$
\left[g_{2}\right]=-6 K_{B}-2 C=6(2 S+(2+m) F)-2 n(S+m F)=(12-2 n) S+(12+6 m-2 n m) F .
$$

This becomes ineffective when $n>3+6 / m$. So, for example, on the Hirzebruch surface $\mathbb{F}_{3}$, tuning an $\mathfrak{s u}(2)$ gauge algebra on $6 \tilde{S}$ would force an additional $\mathfrak{s u}(2)$ to arise on the curve $f_{1}=0$, where $\left[f_{1}\right]=-4 K_{B}-C=2 S+2 F$. Part of this enhancement leads to an enhancement of the $\mathrm{SU}(3)$ gauge factor on the -3 curve $S$, since $\left[f_{1}\right]$ contains $2 S$ as a component. This can be seen (using the Zariski decomposition as in [44]) by noting that $[\sigma] \cdot S=0,[\phi] \cdot S=-2,\left[f_{1}\right] \cdot S=-4$, so the degrees of vanishing of $f, g, \Delta$ on $S$ are $2,3,6$ and the gauge factor on $S$ is enhanced to $\mathrm{SO}(7)$. The remaining part of $\left[f_{1}\right]$ is $\left[f_{1}\right]-2 S=2 F$; this is a reducible curve, giving two $\mathfrak{s u}(2)$ factors each on a copy of $F$, so the total gauge group of the enhanced theory is

$$
G^{\prime}=(\mathrm{SU}(2) \times \mathrm{SU}(2) \times \mathrm{SU}(2) \times \mathrm{SO}(7)) / \mathbb{Z}_{2} .
$$

The matter content includes 6 bifundamentals between the first $\mathrm{SU}(2)$ factor and each of the other $\mathrm{SU}(2)$ factors, and a half-hypermultiplet in the $(\mathbf{2}, \mathbf{8})$ between each of the enhanced $\mathrm{SU}(2)$ factors and the $\mathrm{SO}(7)$ factor (analogous to the matter appearing in the $-2,-3,-2$ non-Higgsable cluster [44]). It is clear that there is no Higgsing of this model possible that would leave the initial $\mathrm{SU}(2)$ factor unbroken. Many other similar models can be realized on other bases with non-Higgsable gauge factors.

Positivity condition and local formulation The condition under which the automatic $\mathfrak{s u}(2)$ enhancement mechanism enhancement must occur is that the divisor $\left[g_{2}\right]=-6 a-2 b$ is ineffective, corresponding to the condition in the $6 \mathrm{D}$ supergravity theory that $-6 a-2 b$ is not in the (closure of the) positivity cone in $\Lambda$. Thus in the case of $\mathfrak{s u}(2)$ a stronger formulation of the conjecture asserts that an $\mathfrak{s u}(2)$ factor with anomaly coefficient $b$, where $-6 a-2 b$ is not in the positivity cone, is not possible unless there is also at least another $\mathfrak{s u}(2)$ factor with anomaly coefficient $b^{\prime}=-4 a-b$. The "local" information about the $\mathfrak{s u}(2)$ algebra is encoded in the quantities $a \cdot b, b \cdot b$, or equivalently $g, b \cdot b$, corresponding in the F-theory picture to the genus (through eq. (3.2)) and self-intersection of the curve $C$ supporting the desired $\mathfrak{s u}(2)$ factor. When $b \cdot b>0$, for example, it is then a sufficient condition for $\left[g_{2}\right]$ to be outside the positivity cone that $-3 a \cdot b<b \cdot b$. As an example of the local version of $\mathfrak{s u}(2)$ enhancement, we can thus conjecture that whenever a theory contains an $\mathfrak{s u}(2)$ algebra with an anomaly coefficient $b$ satisfying $b \cdot b>0, b \cdot b>-3 a \cdot b$ there must also be a nonabelian gauge factor of rank at least one with an anomaly coefficient $b^{\prime}=\left[f_{1}\right]=-4 a-b$, 
or the theory cannot be consistent at least with any F-theory construction. Note that when this $b^{\prime}$ vanishes there is no additional factor, but there is an additional section as discussed in section 4.2.3. When $b^{\prime}$ is not in the (closure of the) positivity cone, then the Weierstrass model develops codimension one $(4,6)$ loci and is inconsistent. This local formulation clearly matches with the examples above; for example, when $T=0,-a=3$, the extra $\mathfrak{s u}(2)$ factor arises when $b=10,11$. Finally, note that on the F-theory side it is possible that $f_{1}$ is reducible, so that the additional gauge factor may be present with a pair (or more) of anomaly coefficients $b_{1}, \ldots, b_{k}$ with $b^{\prime}=b_{1}+\cdots+b_{k}$.

Enhancements that break the theory Note that there are also cases where a similar forced enhancement in the Weierstrass model can lead to codimension one $(4,6)$ loci, which break the F-theory construction; these cases give swampland models that are not addressed by the global version of the automatic enhancement conjecture as stated in section 2 , but which are addressed by the more specific positivity condition and local version stated above. As a simple example of this consider an $\mathfrak{s u}(2)$ factor on the curve $C=4 \tilde{S}$ in the Hirzebruch surface base $\mathbb{F}_{12}$. In this case, $\left[g_{2}\right]=4 S-12 F$ is ineffective and $g_{2}$ vanishes. We furthermore have $\left[f_{1}\right]=4 S+8 F$, so $f_{1}$ vanishes to order 4 on $S$. Since $\phi$ vanishes to order 2 on $S$, there is a codimension one $(4,6)$ singularity on $S$ so no F-theory model is possible. One way of understanding this is that the automatic enhancement on any curve intersecting or containing a -12 curve would naively lead to matter charged under the $\mathrm{E}_{8}$, which is not possible. This model fits with the local version of the $\mathfrak{s u}(2)$ automatic enhancement conjecture as stated above, since $b \cdot b=192,-a \cdot b=56$, so $-3 a \cdot b=168<b \cdot b$, so the local version of the conjecture rules out the theory with gauge group $\mathrm{E}_{8} \times \mathrm{SU}(2)$ with anomaly coefficients $S, 4 \tilde{S}$.

\subsection{2 $\mathfrak{s u}(2) \rightarrow(\mathrm{SU}(2) \times \mathrm{U}(1)) / \mathbb{Z}_{2}$ automatic enhancement}

An interesting further class of related examples occurs with the above $\mathfrak{s u}(2)$ tuning when $\left[g_{2}\right]=0$, so $g_{2}$ is a constant. In this case we get an automatic enhancement to a theory with an additional $\mathrm{U}(1)$ factor. The story is similar to the preceding enhancement that gives the additional $\mathrm{SU}(2)$ factor. One way to understand the resulting $(\mathrm{SU}(2) \times \mathrm{U}(1)) / \mathbb{Z}_{2}$ theory is to consider the theory with gauge group $(\mathrm{SU}(2) \times \mathrm{SU}(2)) / \mathbb{Z}_{2}$ that would arise with $\left[g_{2}\right]=0$, so $g_{2}$ is a constant. In this situation, $\left[f_{1}\right]=-4 a-b=-a$, so $\left[f_{1}\right]$ is a genus one curve; as in the cases above, all fundamental matter in the extra $\mathfrak{s u}(2)$ factor on this curve comes from bifundamentals with the original $\mathfrak{s u}(2)$. Thus, we can Higgs this theory on the adjoint of the second $\mathrm{SU}(2)$, giving a theory with gauge group

$$
G^{\prime}=(\mathrm{SU}(2) \times \mathrm{U}(1)) / \mathbb{Z}_{2},
$$

where all U(1)-charged matter is in the $\mathbf{2}_{1 / 2}$ representation. Thus, the $G^{\prime}$ theory cannot be Higgsed further to a pure $\mathrm{SU}(2)$ theory and this represents another class of automatic enhancements satisfying the conjecture.

This argument is somewhat implicit. A more explicit analysis (see appendix A) shows that when $g_{2}$ is a constant, the Weierstrass model takes the form of a Morrison-Park model [18] with the additional $\mathrm{SU}(2)$ factor on $\{\sigma=0\}$. As for the enhancements described 
above, this story continues to hold in the presence of non-Higgsable gauge factors on more complicated bases, though often the presence of the $\mathrm{U}(1)$ factor simply gives $\mathrm{U}(1)$ charges to the non-Higgsable gauge factors and does not enhance them further. The simplest example of this enhancement arises on the base $\mathbb{P}^{2}$ when an $\mathfrak{s u}(2)$ factor is tuned on a curve of degree $b=9$.

Note that this class of enhancement conditions is similar to that described in section 4.2 .1 , but occurs in conditions where $-3 a=b$ holds identically. This is not really a local condition, as $a \leftrightarrow K_{B}$ involves global information about the geometry in F-theory, as would be expected when abelian $\mathrm{U}(1)$ factors are involved.

\subsection{3 $\mathfrak{s u}(2) \rightarrow \mathrm{SO}(3)$ and massless charge sufficiency}

Note that a similar mechanism to the Automatic Enhancement Conjecture operates when we tune an $\mathfrak{s u}(2)$ algebra on a curve $\sigma$ in the class $-4 a$. In such a situation, $\left[f_{1}\right]=0$ and there is no new gauge factor. But as shown in [19], in F-theory there is automatically a new $\mathbb{Z}_{2}$ element in Mordell-Weil torsion, which gives the gauge group the global structure $\mathrm{SO}(3)=\mathrm{SU}(2) / \mathbb{Z}_{2}$. While this is not an automatic enhancement, this is a case of the Massless Charge Sufficiency condition described in [19]. For example, on the base $\mathbb{P}^{2}$, tuning an $\mathfrak{s u}(2)$ algebra on curves of degrees 9,10,11 leads in F-theory to an automatic enhancement through the mechanism described above, while tuning an $\mathfrak{s u}(2)$ algebra on a curve of degree 12 leads in F-theory to an $\mathrm{SO}(3)$ theory. Thus, if both the Automatic Enhancement Conjecture and the Massless Charge Sufficiency Conjecture are correct in $6 \mathrm{D}$ supergravity, then there would be no swampland for $6 \mathrm{D} \mathcal{N}=1$ theories with $T=0$, gauge group $\mathrm{SU}(2)$, and generic (fundamental and adjoint) matter.

There is a sense in which the Automatic Enhancement Conjecture and the Massless Charge Sufficiency Conjecture are special cases of a common phenomenon. The appearance of the new element of Mordell-Weil torsion in the $\mathfrak{s u}(2) \rightarrow \mathrm{SO}(3)$ situation can in some sense be thought of as adding a new element to the coroot lattice of $G$, within the linear span of $G$. In this sense, the principal difference between the Automatic Enhancement Conjecture and the Massless Charge Sufficiency Conjecture is whether the additional coroot added increases the dimensionality of the gauge algebra or lies in the same vector space. This interpretation is very clear in the F-theory context, where these coroots correspond to homology classes in the compactification space. It would be interesting to investigate whether a similar interpretation can be given directly in $6 \mathrm{D}$ supergravity that would unify these principles.

The local version of this class of conditions is similar to that described in section 4.2.1, but occurs in conditions where $-4 a=b$ holds identically, so $b^{\prime}=\left[f_{1}\right]=0$. (Note that if $-4 a-b=(-a)+(-3 a-b)=0$ then $-3 a-b$ cannot lie in the positivity cone since $-a$ does for all F-theory models.)

\section{3 $T=0$ models with large $\mathfrak{s u}(N)$ gauge algebras at $b=1$}

Similar enhancements occur in models with $\mathfrak{s u}(N)$ gauge algebras for $N>2$. Let us consider models with $T=0$ tensor multiplets, an $\mathfrak{s u}(N)$ gauge group with a $b=1$ anomaly 
coefficient, and the following spectrum of charged hypermultiplets: ${ }^{5}$

$$
3 \times \frac{N(N-1)}{2}+(24-N) \times N .
$$

Such massless spectra are consistent with the anomaly cancellation conditions for $N \leq 24$; for larger $N$, we would require a negative number of fundamental hypermultiplets. Note that these representations (fundamental and two-index antisymmetric), along with the adjoint, are the generic matter representations for $\mathfrak{s u}(n)$.

There are also consistent $T=0$ models with $\mathfrak{s u}(N) \oplus \mathfrak{u}(1)$ gauge algebras and generic matter where the anomaly coefficient for the $\mathfrak{s u}(N)$ factor is $b=1$, at least for $N<19$. For even $N$, the spectrum of charged hypermultiplets is

$3 \times\left(\frac{N(\boldsymbol{N}-\mathbf{1})}{\mathbf{2}}\right)_{0}+\left(12-\frac{N}{2}\right) \times \boldsymbol{N}_{\frac{1}{2}}+\left(12-\frac{N}{2}\right) \times \boldsymbol{N}_{-\frac{1}{2}}+\left(9-\frac{N}{2}\right)\left(12-\frac{N}{2}\right) \times \mathbf{1}_{1}$,

while for odd $N$, the spectrum of charged hypermultiplets is

$3 \times\left(\frac{\boldsymbol{N}(\boldsymbol{N}-\mathbf{1})}{\mathbf{2}}\right)_{-\frac{1}{N}}+\frac{19-N}{2} \times \boldsymbol{N}_{-\frac{N+1}{2 N}}+\frac{29-N}{2} \times \boldsymbol{N}_{\frac{N-1}{2 N}}+\frac{1}{4}(19-N)(23-N) \times \mathbf{1}_{1}$.

Although these spectra naively agree with eq. (4.6) if we ignore the $\mathfrak{u}(1)$ charges, the additional $\mathfrak{u}(1)$ gauge algebra is clearly an important feature. In general, the gauge algebra can be Higgsed down to $\mathfrak{s u}(N)$ by giving VEV's to two hypermultiplets of $\mathbf{1}_{1}$ matter. But if $N$ is either 18 or 19 , there are no $\mathbf{1}_{1}$ hypermultiplets, and the Higgsing is not permitted. The Automatic Enhancement Conjecture therefore implies that the $\mathfrak{s u}(18)$ or $\mathfrak{s u}(19)$ models above should not occur in F-theory, as the gauge algebra should enhance to $\mathfrak{s u}(18) \oplus \mathfrak{u}(1)$ or $\mathfrak{s u}(19) \oplus \mathfrak{u}(1)$.

This enhancement can be seen explicitly if we attempt to construct these models. Because there are no tensor multiplets, the corresponding F-theory models would have $\mathbb{P}^{2}$ as the base of the elliptic fibration. And to obtain an $\mathfrak{s u}(N)$ gauge algebra with a $b=1$ anomaly coefficient, the models should have split $I_{N}$ singularities tuned along a line in this $\mathbb{P}^{2}$. There are known UFD Weierstrass tunings that realize split $I_{N}$ singularities along a divisor $\{\sigma=0\}[42,54]$. The Weierstrass model takes the form

$$
y^{2}=x^{3}+\left(-\frac{1}{3} \Phi^{2}+f_{k} \sigma^{k}\right) x z^{4}+\left(\frac{2}{27} \Phi^{3}-\frac{1}{3} \Phi f_{k} \sigma^{k}+g_{N} \sigma^{N}\right) z^{6}
$$

for $N=2 k$ and

$$
\begin{aligned}
y^{2}=x^{3}+( & \left.-\frac{1}{3} \Phi^{2}+\frac{1}{2} \phi_{0} \psi_{k} \sigma^{k}+f_{k+1} \sigma^{k+1}\right) x z^{4} \\
& +\left(\frac{2}{27} \Phi^{3}-\frac{1}{6} \Phi\left(\phi_{0} \psi_{k}+2 f_{k+1} \sigma\right) \sigma^{k}+\frac{1}{4} \psi_{k}^{2} \sigma^{2 k}+g_{N} \sigma^{N}\right) z^{6}
\end{aligned}
$$

\footnotetext{
${ }^{5}$ For $N=3$, the hypermultiplet spectrum would consist of 24 fundamental hypermultiplets, but the $N=3$ case is not of significant interest here.
} 
for $N=2 k+1$, where $^{6}$

$$
\Phi=\frac{1}{4} \phi_{0}^{2}+\phi_{1} \sigma
$$

These models support hypermultiplets in the antisymmetric and fundamental representations. ${ }^{7}$ Thus, the natural strategy for constructing our desired $\mathfrak{s u}(N)$ models is to apply these tunings in an elliptic fibration over $\mathbb{P}^{2}$ with $[\sigma]=H$. The other parameters then have the following divisor classes:

$$
\left[\phi_{0}\right]=3 H \quad\left[\phi_{1}\right]=5 H \quad\left[\psi_{k}\right]=(9-k) H \quad\left[f_{i}\right]=(12-i) H \quad\left[g_{j}\right]=(18-j) H .
$$

For $N \leq 17$ and $N=24$, these tunings give us the desired $\mathfrak{s u}(N)$ models without any enhancements of the gauge algebra. ${ }^{8}$ But when $N=18,\left[g_{N}\right]$ is trivial, and $g_{N}$ is automatically a perfect square. If we replace $g_{N}$ with $\gamma^{2}$, the elliptic fibration described by eq. (4.9) admits a rational section

$$
[\hat{x}: \hat{y}: \hat{z}]=\left[\frac{1}{3} \Phi: \gamma \sigma^{9}: 1\right] .
$$

The gauge algebra is therefore $\mathfrak{s u}(18) \oplus \mathfrak{u}(1)$, and one can confirm with the Katz-Vafa method [55] and the proposals in [56] that the charged hypermultiplet spectrum agrees with eq. (4.7). When $N=19,\left[g_{N}\right]$ is ineffective, and $g_{N}$ is essentially zero. After setting $N$ to 19 and $g_{N}$ to 0 , the elliptic fibration described by eq. (4.10) admits the rational section

$$
[\hat{x}: \hat{y}: \hat{z}]=\left[\frac{1}{3} \Phi: \frac{1}{2} \psi_{9} \sigma^{9}: 1\right],
$$

implying that the gauge algebra is enhanced to $\mathfrak{s u}(19) \oplus \mathfrak{u}(1)$. And by the Katz-Vafa method [55] and the proposals in [56], one can again verify that the charged hypermultiplet spectrum agrees with eq. (4.8). Clearly, we see the enhancements expected from the Automatic Enhancement Conjecture in these two models.

Enhancements also occur for $N$ larger than 19, as previously observed in [42, 43]. ${ }^{9}$ The enhancements are summarized in table 1 . If we use eq. (4.9) to construct the $\mathfrak{s u}(20)$ and $\mathfrak{s u}(22)$ models, the divisor class $\left[g_{N}\right]$ becomes ineffective, and the gauge algebras enhance to $\mathfrak{s u}(20) \oplus \mathfrak{s u}(2)$ or $\mathfrak{s u}(22) \oplus \mathfrak{s u}(2)$ respectively. (The generating section in eq. (4.13) becomes torsional [57], and the gauge group is $(\mathrm{SU}(N) \times \mathrm{SU}(2)) / \mathbb{Z}_{2}$.) While the extra $\mathfrak{s u}(2)$ algebra in both cases could be Higgsed by giving VEVs to two $(\mathbf{1}, \mathbf{2})$ hypermultiplets, the $\mathfrak{s u}(20) \oplus \mathfrak{s u}(2)$ and $\mathfrak{s u}(22) \oplus \mathfrak{s u}(2)$ spectra in table 1 do not have this matter. Thus, the appearance of this extra $\mathfrak{s u}(2)$ algebra agrees with the Automatic Enhancement Conjecture.

Meanwhile, if we use eq. (4.10) to construct the $\mathfrak{s u}(21)$ and $\mathfrak{s u}(23)$ models, the divisor class $\left[\psi_{k}\right]$ becomes ineffective, and the gauge algebras enhance to $\mathfrak{s u}(22) \oplus \mathfrak{s u}(2)$ and $\mathfrak{s u}(24)$,

\footnotetext{
${ }^{6} \operatorname{In}[42], \Phi$ is defined as $\frac{1}{4} \phi_{0}^{2}+\phi_{1} \sigma+\ldots+\phi_{k-1} \sigma^{k-1}$. However, one can remove the $\phi_{i>1}$ parameters by the redefinition $\phi_{1} \rightarrow \phi_{1}-\left(\phi_{2} \sigma+\ldots+\phi_{k-1} \sigma^{k-2}\right)$.

${ }^{7}$ If $\{\sigma=0\}$ is a curve of genus greater than 0 , there may also be adjoint hypermultiplets, although this possibility is not important here.

${ }^{8}$ The $N=24$ model, however, is an example of the Massless Charge Sufficiency Conjecture, as an additional torsional section appears.

${ }^{9}$ Because it is somewhat more difficult to find extra rational sections, these previous works did not point out the additional $\mathfrak{u}(1)$ algebras for $N=18$ and 19 .
} 


\begin{tabular}{|ccc|}
\hline Expected Gauge Algebra & Enhanced Gauge Algebra & Matter Spectrum \\
\hline $\mathfrak{s u}(18)$ & $\mathfrak{s u}(18) \oplus \mathfrak{u}(1)$ & $3 \times \mathbf{1 5 3}_{0}+3 \times \mathbf{1 8}_{1 / 2}+3 \times \mathbf{1 8}_{-1 / 2}$ \\
$\mathfrak{s u}(19)$ & $\mathfrak{s u}(19) \oplus \mathfrak{u}(1)$ & $3 \times \mathbf{1 7 1}_{-1 / 19}+5 \times \mathbf{1 9}_{9 / 19}$ \\
$\mathfrak{s u}(20)$ & $\mathfrak{s u}(20) \oplus \mathfrak{s u}(2)$ & $3 \times(\mathbf{1 9 0}, \mathbf{1})+2 \times(\mathbf{2 0}, \mathbf{2})$ \\
$\mathfrak{s u}(21)$ & $\mathfrak{s u}(22) \oplus \mathfrak{s u}(2)$ & $3 \times(\mathbf{2 3 1}, \mathbf{1})+(\mathbf{2 2}, \mathbf{2})$ \\
$\mathfrak{s u}(22)$ & $\mathfrak{s u}(22) \oplus \mathfrak{s u}(2)$ & $3 \times(\mathbf{2 3 1}, \mathbf{1})+(\mathbf{2 2}, \mathbf{2})$ \\
$\mathfrak{s u}(23)$ & $\mathfrak{s u}(24)$ & $3 \times \mathbf{2 7 6}$ \\
$\mathfrak{s u}(24)$ & $\mathfrak{s u}(24)$ & $3 \times \mathbf{2 7 6}$ \\
\hline
\end{tabular}

Table 1. Enhanced gauge algebras observed when attempting to tune various expected gauge algebras along a divisor of class $H$ on $\mathbb{P}^{2}$, along with the observed matter spectrum.

respectively. Neither of these enhanced models has the requisite matter to Higgs the gauge algebra to $\mathfrak{s u}(21)$ or $\mathfrak{s u}(23)$. While one can give VEVs to $(\mathbf{2 2 , 2})$ matter to break $\mathfrak{s u}(22) \oplus$ $\mathfrak{s u}(2)$ to $\mathfrak{s u}(21)$ [58], one would need at least two hypermultiplets of $(\mathbf{2 2}, \mathbf{2})$ matter to satisfy the D-term constraints [59]. And the $\mathfrak{s u}(24)$ model, which supports only antisymmetric hypermultiplets, lacks the fundamental matter necessary to Higgs the gauge algebra down to $\mathfrak{s u}(23)$. Therefore, the observed enhancements when attempting to construct the $\mathfrak{s u}(21)$ and $\mathfrak{s u}(23)$ models agree with the expectations of the Automatic Enhancement Conjecture.

The local version of this class of enhancements states that for any element $b \in \Lambda$ satisfying $b \cdot b=1, g=0(-a \cdot b=3)$, an isolated gauge algebra of $\mathfrak{s u}(18)$ or more is automatically enhanced according to table 1 .

\subsection{Other $\mathrm{SU}(N)$ examples}

In the last two subsections we have focused on enhancements of the algebra $\mathfrak{s u}(2)$ with general anomaly coefficients and enhancements of the algebra $\mathfrak{s u}(n)$ at large $n$ on anomaly coefficients $b \cdot b=1$, where $g=0$. We briefly discuss some more general $\mathfrak{s u}(n)$ examples

\subsubsection{Enhancements of $\mathfrak{s u}(3)$ and beyond for general $b$}

A similar enhancement to that described in section 4.2.1 occurs for $\mathfrak{s u}(3)$. When the coefficients in classes $\left[f_{2}\right]=-4 a-2 b$ and $\left[g_{3}\right]=-6 a-3 b$ in a $\sigma$ expansion of $f, g$ for the general $\mathrm{SU}(3)$ form $[19,42]$ are not effective, the discriminant acquires an additional cubic factor $\psi_{1}^{3}$ where $\left[\psi_{1}\right]=-3 a-b$. This gives an enhancement $\mathfrak{s u}(3) \rightarrow \mathfrak{s u}(3) \oplus \mathfrak{s u}(3)$. Just as there is $\mathbb{Z}_{2}$ torsion in the $\mathfrak{s u}(2) \rightarrow \mathfrak{s u}(2) \oplus \mathfrak{s u}(2)$ situations of section 4.2.1, there is automatically $\mathbb{Z}_{3}$ Mordell-Weil torsion in these cases [19], so the global gauge group becomes $G^{\prime}=(\mathrm{SU}(3) \times \mathrm{SU}(3)) / \mathbb{Z}_{3}$. Again, the second $\mathrm{SU}(3)$ factor has no adjoints and all fundamentals/antifundamentals are jointly charged, so the second $\mathrm{SU}(3)$ factor cannot be Higgsed without breaking the original SU(3). For $T=0$, this enhancement occurs for the values $b=7,8$. At $b=6, f_{2}, g_{3}$ become constants and the algebra is enhanced by $\mathfrak{u}(1) \oplus \mathfrak{u}(1)$, by the same logic that led to eq. (4.5). The local version of this analysis is that when $b \cdot b>0, b \cdot b>-2 a \cdot b$ we get an extra $\mathfrak{s u}(3)$ factor on $-3 a-b$.

We now consider $\mathfrak{s u}(4)$. In general what is needed for automatic enhancement to an extra SU(2) factor with generic (fundamental, adjoint) matter that cannot be Higgsed down 
is that the extra factor must have an anomaly coefficient $b^{\prime}$ giving $g^{\prime}=0$ (for the absence of adjoints) and the automatically enhanced group must provide all fundamental $\mathrm{SU}(2)$ matter. The number of fundamental matter fields in this situation is $b^{\prime} \cdot\left(-8 a-2 b^{\prime}\right)$ from anomaly cancellation. For an $\mathfrak{s u}(4)$ with anomaly coefficient $b$ to experience enhancement to $\mathfrak{s u}(4) \oplus \mathfrak{s u}(2)$ we must then have $4 b \cdot b^{\prime}=b^{\prime} \cdot\left(-8 a-2 b^{\prime}\right)$. This can certainly occur, most simply when $2 b=-4 a-b^{\prime}$. For example, this occurs when $T=0$ where $b=5, b^{\prime}=2$. In general, this will occur when $-3 a-2 b$ is not positive but $-4 a-2 b$ is positive; it is not hard to confirm in the universal Weierstrass model for an $\mathfrak{s u}(4)$ with generic matter that this enhancement occurs under these conditions, where $f_{3}=g_{4}=0$.

We leave further exploration of automatic enhancement of this type for gauge factors with general anomaly coefficients to further work.

\subsubsection{Enhancements of $\mathfrak{s u}(N)$ for $g=0$, small $b \cdot b$}

We now focus on more general enhancements of $\mathfrak{s u}(N)$ for larger $N$, restricting to the cases where $g=0$. For $\mathfrak{s u}(N)$ models with $g=0, b \cdot b=2$, the largest value of $N$ allowed by the anomaly cancellation conditions is $N=16$. Automatic enhancement should occur for at least $N \geq 13$ because of the requirement that $(-6 a-N b) \cdot b$ must be non-negative for $\left[g_{N}\right]=-6 a-N b$ to be effective. But automatic enhancement can also occur for some $N$ less than 13 depending on the base of the F-theory model in question, since $-6 a-N b$ can be outside the positivity cone even if $(-6 a-N b) \cdot b \geq 0$. For instance, let us restrict attention to $T=1$ models, which are constructed using the Hirzebruch surfaces $\mathbb{F}_{m}$ as bases. To ensure that the divisor supporting the $\mathfrak{s u}(N)$ algebra is irreducible and has selfintersection $+2, m$ should be 0 or 2 . The automatic enhancements, which are the same for both of these cases, are summarized in table 2 .

The pattern of enhancements may be different for $b \cdot b=2$ divisors on other bases. For instance, consider a base $B$ found by blowing up $\mathbb{F}_{2}$ at the intersection of the -2 curve and a 0 curve, and imagine tuning an $\mathfrak{s u}(N)$ algebra on a +2 curve $\{\sigma=0\}$. There are no automatic enhancements for $N \leq 9$ on $B$, apart from a relatively uninteresting $\mathfrak{s u}(3) \mathrm{NHC}$ on the -3 curve. For $N=10,\left[g_{10}\right]=-6 K_{B}-10[\sigma]$ is effective and nontrivial, but the only holomorphic sections of $\left[g_{10}\right]$ are perfect squares. The gauge algebra should therefore admit an extra $\mathfrak{u}(1)$ factor. In fact, the full gauge algebra is $\mathfrak{s u}(10) \oplus \mathfrak{s u}(3) \oplus \mathfrak{s u}(2) \oplus \mathfrak{u}(1)$, and the charged hypermultiplet spectrum is

$$
4 \times(\mathbf{4 5}, \mathbf{1}, \mathbf{1})_{0}+1 \times(\mathbf{1 0}, \mathbf{1}, \mathbf{2})_{0}+5 \times(\mathbf{1 0}, \mathbf{1}, \mathbf{1})_{\frac{1}{2}}+5 \times(\mathbf{1 0}, \mathbf{1}, \mathbf{1})_{-\frac{1}{2}} .
$$

This spectrum satisfies the conditions of the Automatic Enhancement Conjecture, as one cannot Higgs away the $\mathfrak{s u}(2)$ or $\mathfrak{u}(1)$ factors without disturbing the $\mathfrak{s u}(10)$ algebra. Tuning $\mathfrak{s u}(10)$ on the $b \cdot b=2$ curve in $B$ should therefore lead to an automatic enhancement as observed, even though a similar $\mathfrak{s u}(10)$ enhancement does not occur in the $T=1$ models above. Locally, this enhancement can be understood as being forced from the intersection of the +2 curve with a curve of self-intersection -1 , and can be understood using purely local analysis as in [43]. Likewise, $\mathfrak{s u}(11)$ and $\mathfrak{s u}(12)$ experience automatic enhancements when tuned on the +2 curve of $B$, yet they can be obtained without enhancements when $T=1$. 


\begin{tabular}{|ccc|}
\hline Expected Gauge Algebra & Enhanced Gauge Algebra & Matter Spectrum \\
\hline $\mathfrak{s u}(12)$ & $\mathfrak{s u}(12) \oplus \mathfrak{u}(1)$ & $4 \times \mathbf{6 6}_{0}+4 \times \mathbf{1 2}_{\frac{1}{2}}+4 \times \mathbf{1 2}_{-\frac{1}{2}}$ \\
$\mathfrak{s u}(13)$ & $\mathfrak{s u}(13) \oplus \mathfrak{u}(1)$ & $4 \times \mathbf{7 8}_{-\frac{1}{13}}+6 \times \mathbf{1 3}_{\frac{6}{13}}$ \\
$\mathfrak{s u}(14)$ & $\mathfrak{s u}(14) \oplus \mathfrak{s u}(2)$ & $4 \times(\mathbf{9 1}, \mathbf{1})+2 \times(\mathbf{1 4}, \mathbf{2})$ \\
$\mathfrak{s u}(15)$ & $\mathfrak{s u}(16)$ & $4 \times \mathbf{1 2 0}$ \\
$\mathfrak{s u}(16)$ & $\mathfrak{s u}(16)$ & $4 \times \mathbf{1 2 0}$ \\
\hline
\end{tabular}

Table 2. Automatic enhancements for $T=1$ models with $\mathfrak{s u}(N)$ gauge algebras tuned on divisors of genus $g=0$ and self-intersection $b \cdot b=2$.

For $g=0$ and $b \cdot b=3$, the largest value of $N$ allowed by the anomaly cancellation is 13. Based on the condition for $(-6 a-N b) \cdot b$, automatic enhancements should occur at least for $N \geq 11$. Again, the actual pattern of enhancements depends on the base of the F-theory model. When $T=1$, one can find irreducible curves with self-intersection +3 on $\mathbb{F}_{1}$ and $\mathbb{F}_{3}$. On $\mathbb{F}_{1}$, there is no enhancement for $N \leq 8$, and the enhanced gauge algebras are $\mathfrak{s u}(9) \oplus \mathfrak{s u}(2)$ for $N=9, \mathfrak{s u}(10) \oplus \mathfrak{s u}(2) \oplus \mathfrak{s u}(2)$ for $N=10$, and $\mathfrak{s u}(12) \oplus \mathfrak{s p}(2)$ for $N=11$ and $N=12 .{ }^{10}$ On $\mathbb{F}_{3}$, there is no enhancement for $N \leq 9$ apart from the usual $\mathfrak{s u}(3)$ non-Higgsable cluster. The enhanced gauge algebras are $\mathfrak{s u}(10) \oplus \mathfrak{u}(1) \oplus \mathfrak{s u}(3)$ for $N=10, \mathfrak{s u}(11) \oplus \mathfrak{u}(1) \oplus \mathfrak{s u}(3)$ for $N=11$, and $\mathfrak{s u}(12) \oplus \mathfrak{s u}(2) \oplus \mathfrak{s u}(2) \oplus \mathfrak{s o}(7)$ for $N=12,{ }^{11}$ with the last gauge algebra representing that supported on the -3 curve. For both of these bases, attempting to obtain an $\mathfrak{s u}(13)$ gauge algebra using the standard tuning of eq. (4.10) produces an invalid elliptic fibration with singular fibers everywhere on the base. While we do not discuss the matter spectra for brevity, they are all consistent with the Automatic Enhancement Conjecture.

\subsection{Examples with $\mathfrak{s p}(N)$ and $\mathfrak{s o}(N)$ algebras}

We can immediately obtain $\mathfrak{s p}(m)$ examples of automatic enhancement by Higgsing the $\mathfrak{s u}(2 m)$ models of section 4.3. If we give a VEV to matter in the antisymmetric representation of $\mathfrak{s u}(2 m)$, we obtain an $\mathfrak{s u}(m)$ gauge algebra with the same anomaly coefficient $b$. From an F-theory perspective, the resulting $\mathfrak{s p}(m)$ model is given by taking the $\mathfrak{s u}(2 m)$ construction of eq. (4.9) and ignoring the tuning of $\Phi$ in eq. (4.11). The $\mathfrak{s u}(2 m)$ models of section 4.3 have enough antisymmetric hypermultiplets to perform this Higgsing. However, these hypermultiplets are uncharged under any additional $\mathfrak{s u}(2)$ or $\mathfrak{u}(1)$ factor appearing due to automatic enhancements, and the $\mathfrak{s u}(2 m) \rightarrow \mathfrak{s p}(m)$ breaking does not introduce new hypermultiplets that would let us Higgs the extra gauge factors without disturbing the $\mathfrak{s p}(m)$ algebra. This fact provides us with $T=0$ examples of automatic enhancement with $\mathfrak{s p}(m)$ gauge algebras and $b=1$ anomaly coefficients.

When $m=9$, for instance, the conjecture implies that $\mathfrak{s p}(9)$ experiences an automatic enhancement to $\mathfrak{s p}(9) \oplus \mathfrak{u}(1)$ with the following charged hypermultiplet spectrum:

$$
2 \times 152_{0}+3 \times 18_{1 / 2}+3 \times 18_{-1 / 2} .
$$

\footnotetext{
${ }^{10}$ We use conventions where $\mathfrak{s p}(2)$ is supported along non-split $I_{4}$ loci.

${ }^{11}$ Since it can be difficult to find rational sections, the enhanced gauge algebras may actually have $\mathfrak{u}(1)$ factors beyond those listed here, which we were able to identify easily.
} 
This enhancement can be seen in the $\mathfrak{s p}(9)$ Weierstrass model produced by the procedure described above: the class $\left[g_{18}\right]$ is trivial, leading to an additional section of the elliptic fibration. Similarly, $\mathfrak{s p}(10)$ and $\mathfrak{s p}(11)$ experience automatic enhancements to $\mathfrak{s p}(10) \oplus \mathfrak{s u}(2)$ and $\mathfrak{s p}(11) \oplus \mathfrak{s u}(2)$, respectively. While the $\mathfrak{s p}(12)$ model does not experience automatic enhancement, the Weierstrass model has an extra torsional section, in agreement with the Massless Charge Sufficiency Conjecture as noted in [19].

There are also $\mathfrak{s o}(N)$ examples with automatic enhancement. For example, consider a $T=0$ model with an $\mathfrak{s o}(10)$ gauge algebra and $b=4$ anomaly coefficient. The charged hypermultiplet spectrum for this model is

$$
3 \times \mathbf{4 5}+8 \times \mathbf{1 6}+4 \times \mathbf{1 0} .
$$

However, there is also a consistent $\mathfrak{s o}(10) \oplus \mathfrak{u}(1)$ model with anomaly coefficients $b=4$, $\tilde{b}=1$ and a charged hypermultiplet spectrum of the form

$$
3 \times 45_{0}+8 \times 16_{\frac{1}{4}}+4 \times 10_{\frac{1}{2}} .
$$

The Automatic Enhancement Conjecture implies that we should see an $\mathfrak{s o}(10) \rightarrow \mathfrak{s o}(10) \oplus$ $\mathfrak{u}(1)$ enhancement. In fact, this can be seen in the $\mathfrak{s o}(10)$ Weierstrass tuning

$$
y^{2}=x^{3}+\sigma^{2}\left(-\frac{1}{48} \Phi^{2}+f_{3} \sigma\right)+\sigma^{3}\left(\frac{1}{864} \Phi^{3}+\left(\gamma^{2}-\frac{1}{12} f_{3} \Phi\right) \sigma+g_{5} \sigma^{2}\right) .
$$

The $\mathfrak{s o}(10)$ algebra is tuned along $\{\sigma=0\}$, so $[\sigma]$ should be $4 H$ to obtain the correct $\mathfrak{s o}(10)$ hypermultiplet spectrum. But the divisor class $\left[g_{5}\right]=-6 K_{B}-5[\sigma]=-2 H$ is then ineffective, forcing $g_{5}$ to be 0 . In turn, the elliptic fibration acquires an additional rational section of the form

$$
[\hat{x}: \hat{y}: \hat{z}]=\left[\frac{1}{12} \sigma \Phi: \gamma \sigma^{2}: 1\right],
$$

demonstrating the expected appearance of the $\mathfrak{u}(1)$ gauge algebra.

\section{Abelian examples}

The Automatic Enhancement Conjecture also suggests that abelian gauge symmetries in certain models are forced to enhance. In fact, the conjecture is particularly powerful in these settings, as it can explain why infinite families of apparently consistent supergravity models with abelian gauge symmetries do not occur in F-theory. We first describe cases of the Automatic Enhancement Conjecture with U(1) gauge group and generic $(q=1,2)$ matter, and then focus on two types of infinite families that include exotic U(1) charges: those with $\mathrm{U}(1)$ gauge groups, and an infinite family with an $\mathfrak{s u}(13) \oplus \mathfrak{u}(1)$ gauge algebra.

\subsection{U(1) models with generic matter}

For a gauge group $\mathrm{U}(1)$, the generic matter representations are those with charges $q=$ 1,2 [25]. Weierstrass models for F-theory constructions of $\mathrm{U}(1)$ theories with these generic matter charges were constructed by Morrison and Park [18]. The explicit form of these Weierstrass models is given in eq. (A.2), and it was shown that these models are universal in 
the sense that they capture all the uncharged moduli, at least over simple bases, in [60,61]. There is a class of situations where the generic U(1) model with certain anomaly coefficients satisfies the conditions of the Automatic Enhancement Conjecture. These situations are closely related to the $\mathfrak{s u}(2)$ models described in section 4.2.1. Consider a 6D supergravity theory with gauge algebra $\mathfrak{s u}(2) \oplus \mathfrak{s u}(2)$, and anomaly coefficients $b, b^{\prime}$ satisfying $b+b^{\prime}=$ $-4 a$. In such a situation, the spectrum consists of $b \cdot b^{\prime}$ bifundamental matter fields and $g, g^{\prime}$ adjoint fields for the two $\mathfrak{s u}(2)$ factors, where $g, g^{\prime}$ are given by eq. (3.2) using $b, b^{\prime}$ respectively. When $g>0$, the first $\mathfrak{s u}(2)$ factor can be broken down to $\mathfrak{u}(1)$ by Higgsing on an adjoint field. When $g^{\prime}=0$, however, the second $\mathfrak{s u}(2)$ factor cannot be broken through Higgsing while preserving the first $\mathfrak{u}(1)$ factor. This gives a situation with gauge algebra $\mathfrak{u}(1) \oplus \mathfrak{s u}(2)$ and matter charged only in the $\mathbf{1}_{2}, \mathbf{2}_{1}$ representations. When such a theory can be constructed in F-theory, the conditions of the Automatic Enhancement Conjecture are satisfied, so we expect that the theory with only gauge algebra $\mathfrak{u}(1)$ and anomaly coefficient $\tilde{b}=2 b$ cannot be realized in F-theory.

The simplest example of this is in the case of no tensor multiplets $(T=0)$. There are anomaly-consistent $\mathrm{U}(1)$ models with anomaly coefficient $\tilde{b}=6,8, \ldots, 24$ and spectrum $\tilde{b}(24-\tilde{b}) \times( \pm \mathbf{1}), \tilde{b}(\tilde{b}-6) / 4 \times( \pm \mathbf{2})$. Naively, these come from Higgsing the anomalyconsistent $\mathfrak{s u}(2)$ model with $b=\tilde{b} / 2$. As we have seen, however, in section 4.2 .1 , the $\mathfrak{s u}(2)$ models with $b=10,11$ experience automatic enhancement, and from the above analysis the same is expected for the $\mathfrak{u}(1)$ models with $\tilde{b}=20,22$.

Indeed, we can see explicitly from the form of the Morrison-Park Weierstrass model eq. (A.2) that for the cases $\tilde{b}=20,22$, which correspond to choosing classes $\left[c_{3}\right]=\tilde{b} / 2=$ 10,11 , the class $\left[c_{0}\right]$ becomes ineffective and there is an enhancement to an additional $\mathfrak{s u}(2)$ factor on the class $\left[c_{1}\right]$. This enhancement was also discussed from different points of view in $[18,61]$. More generally, whenever we have a $\mathfrak{u}(1)$ theory with generic matter and an anomaly coefficient $\tilde{b}$ such that $-6 a-\tilde{b}$ is outside the positivity cone but $-8 a-\tilde{b}$ is in the (closure of the) cone, we have a similar situation where the theory suffers automatic enhancement to $\mathfrak{u}(1) \oplus \mathfrak{s u}(2)$. In the F-theory construction, it can be confirmed that the global structure of the group in such situations is $(\mathrm{U}(1) \times \mathrm{SU}(2)) / \mathbb{Z}_{2}[19]$.

There are also similar situations when $-6 a-\tilde{b}=0$, in which case the enhancement is to a $\mathrm{U}(1) \times \mathrm{U}(1)$ theory, as can be seen again directly from the Morrison-Park form, and when $\tilde{b}=-8 a$, which naively gives a $\mathfrak{u}(1)$ model with only even charges, but where the presence of an additional section in the Morrison-Park model makes this equivalent to the model with only charges $( \pm \mathbf{1})$, in accord with the Massless Charge Sufficiency Conjecture [19]. Note that the original Morrison-Park generating section in the enhanced $\mathrm{U}(1) \times \mathrm{U}(1)$ theory when $-6 a=\tilde{b}$ is the "diagonal" sum of the two $\mathrm{U}(1)$ generators giving the charge lattice generated by $( \pm \mathbf{1}, \mathbf{0})$ and $(\mathbf{0}, \pm \mathbf{1})$. These cases play a further role as a special case in the analysis of the following subsection.

\section{$5.2 \mathrm{U}(1)$ infinite families}

We now turn to some families of $\mathrm{U}(1)$ models with exotic charges $q>2$. It was shown in [25] that there is an infinite family of $\mathrm{U}(1)$ models with no tensor multiplets that satisfy the $6 \mathrm{D}$ anomaly cancellation conditions and other known low-energy constraints. The models 
in this family have $\mathrm{U}(1)$ height

$$
\tilde{b}=6\left(q^{2}+q r+r^{2}\right)
$$

and charged hypermultiplet spectra of the form ${ }^{12}$

$$
54 \times( \pm \boldsymbol{q})+54 \times( \pm \boldsymbol{r})+54 \times( \pm(\boldsymbol{q}+\boldsymbol{r}))
$$

for $q, r \in \mathbb{Z}$. From now on, we ignore situations where either $q, r$, or $q+r$ is 0 ; after normalizing charges, these situations correspond to a relatively uninteresting model known to occur in F-theory [18] whose charged hypermultiplet spectrum has only $108( \pm \mathbf{1})$ hypermultiplets. We also assume that $q, r$ are relatively prime, so that these charges generate the full possible charge lattice and therefore satisfy the Massless Charge Sufficiency Conjecture [19]. ${ }^{13}$ Because the number of topologically distinct elliptically fibered Calabi-Yau threefolds is finite, only a finite number of these supergravity models can be realized in F-theory. This family therefore presents an infinite number of swampland candidates, but there has been no proposal as to which precise subset of these models lie in the F-theory swampland, let alone why. And since the models in this family support arbitrarily large $\mathrm{U}(1)$ charges, this family is relevant for questions regarding which $\mathrm{U}(1)$ charges occur in F-theory (see, e.g., [41, 63-66]).

However, there is also a model with zero tensor multiplets, a $\mathrm{U}(1) \times \mathrm{U}(1)$ gauge group, and a relatively simple spectrum of charged hypermultiplets: ${ }^{14}$

$$
54 \times(\mathbf{1}, \mathbf{- 1})+54 \times(\mathbf{1}, \mathbf{0})+54 \times(\mathbf{0}, \mathbf{1}) .
$$

The anomaly coefficient matrix for the $\mathrm{U}(1) \times \mathrm{U}(1)$ gauge group is

$$
\tilde{b}=\left(\begin{array}{cc}
6 & -3 \\
-3 & 6
\end{array}\right) .
$$

This model appears repeatedly in this section, and unless otherwise specified, any reference to a $\mathrm{U}(1) \times \mathrm{U}(1)$ model refers to this specific model. Let us refer to the first $\mathrm{U}(1)$ factor as $\mathrm{U}(1)_{a}$ and the second $\mathrm{U}(1)$ factor as $\mathrm{U}(1)_{b}$. We are also free to use a different basis $\mathrm{U}(1)_{c} \times \mathrm{U}(1)_{d}$, such that ${ }^{15}$

$$
\left(\begin{array}{l}
q_{c} \\
q_{d}
\end{array}\right)=\left(\begin{array}{cc}
q & -r \\
0 & 1
\end{array}\right)\left(\begin{array}{l}
q_{a} \\
q_{b}
\end{array}\right) .
$$

The charge spectrum in this new basis is

$$
54 \times(\boldsymbol{q}+\boldsymbol{r}, \mathbf{- 1})+54 \times(\boldsymbol{q}, \mathbf{0})+54 \times(\boldsymbol{r}, \mathbf{1}),
$$

\footnotetext{
${ }^{12}$ The sign of $\mathrm{U}(1)$ charge for a charged hypermultiplet is unimportant, as a $( \pm \boldsymbol{Q})$ hypermultiplet contains fields with $\mathrm{U}(1)$ charges $+Q$ and $-Q$.

${ }^{13}$ Tarazi and Vafa have made the stronger conjecture [62] that there must always be a massless field of charge $( \pm \mathbf{1})$ in a $6 \mathrm{D} \mathrm{U}(1)$ supergravity theory of this type, which would be violated by many of these theories, but there would still be an infinite family with $q=1, r$ prime compatible even with this stronger conjecture.

${ }^{14} \mathrm{~A}\left(\boldsymbol{Q}_{\boldsymbol{a}}, \boldsymbol{Q}_{\boldsymbol{b}}\right)$ hypermultiplet is equivalent to $\left(-\boldsymbol{Q}_{\boldsymbol{a}},-\boldsymbol{Q}_{\boldsymbol{b}}\right)$ hypermultiplet, as both contain fields with charges $\left(Q_{a}, Q_{b}\right)$ and $\left(-Q_{a},-Q_{b}\right)$.

${ }^{15}$ This transformation is not valid if $q=0$, but as mentioned above this situation is uninteresting.
} 
and the anomaly coefficient matrix is

$$
\left(\begin{array}{cc}
6\left(q^{2}+q r+r^{2}\right) & -3(q+2 r) \\
-3(q+2 r) & 6
\end{array}\right) .
$$

Although $\mathrm{U}(1)_{c}$ corresponds to the $\mathrm{U}(1)$ seen in the infinite family, there are still two $\mathrm{U}(1)$ factors in this model. One could imagine obtaining a model in the infinite family by Higgsing $\mathrm{U}(1)_{d}$ in a way that preserves $\mathrm{U}(1)_{c}$. This Higgsing would entail giving a VEV to $(\mathbf{0}, \mathbf{1})$ matter in the $\mathrm{U}(1)_{c} \times \mathrm{U}(1)_{d}$ basis, but this matter is not present in the spectrum unless $r$ or $r+q$ is 0 . The Automatic Enhancement Conjecture would then imply that if we attempt to construct any $\mathrm{U}(1)$ model in the family with $r, q, r+q \neq 0$, the model is forced to obtain an extra $\mathrm{U}(1)$, and we end up with the $\mathrm{U}(1) \times \mathrm{U}(1)$ model.

The $\mathrm{U}(1) \times \mathrm{U}(1)$ model has an F-theory realization described in appendix B. Geometrically, $\mathrm{U}(1)_{a}$ and $\mathrm{U}(1)_{b}$ correspond to the generating sections $\hat{s}_{Q}$ and $\hat{s}_{R}$, while $\mathrm{U}(1)_{c}$ corresponds to the linear combination $q \hat{s}_{Q}-r \hat{s}_{R}$ under elliptic curve addition. The natural way to test the Automatic Enhancement Conjecture is to try constructing the models in the infinite family using the known F-theory $\mathrm{U}(1)$ constructions and to show that we end up with the $\mathrm{U}(1) \times \mathrm{U}(1)$ F-theory model. Because the known $\mathrm{U}(1)$ constructions in F-theory admit only relatively small charges, we will only be able to test the conjecture for small $r$ and $q$. Still, we see clear evidence of the expected enhancement in all the cases we consider. When we try to use these constructions to obtain $\mathrm{U}(1)$ models in the infinite family, the divisor classes of various parameters become either ineffective or trivial. As a result, the Mordell-Weil rank enhances, and the section that seems to support the correct $\mathrm{U}(1)$ charges becomes a combination of $\hat{s}_{Q}$ and $\hat{s}_{R}$.

$r=q=1$ When $r$ and $q$ are both 1, the desired spectrum of charged hypermultiplets for the $\mathrm{U}(1)$ model takes the form

$$
108 \times( \pm \mathbf{1})+54 \times( \pm \mathbf{2}) .
$$

Since the U(1) model only has matter with charges $q= \pm 1$ and \pm 2 , the Weierstrass model should be in the Morrison-Park form (A.2). We take the base of the elliptic fibration to be $\mathbb{P}^{2}$. For $\tilde{b}=6\left(q^{2}+q r+r^{2}\right)=18$, the parameters in Morrison-Park form would have divisor classes

$$
[\hat{b}]=6 H, \quad\left[c_{3}\right]=9 H, \quad\left[c_{2}\right]=6 H, \quad\left[c_{1}\right]=3 H, \quad\left[c_{0}\right]=0 H .
$$

This is precisely an example of the enhancement structure described in the last paragraph of the preceding subsection. The divisor class $\left[c_{0}\right]$ is trivial, and $c_{0}$ can freely be taken to be a perfect square. But if $c_{0}$ is a perfect square, the gauge group enhances to $\mathrm{U}(1) \times \mathrm{U}(1)$ [61] . One can verify the presence of this additional U(1) by counting moduli and comparing to the expectations from the gravitational anomaly cancellation conditions [17]. Additionally, we can map the Morrison-Park form with trivial $\left[c_{0}\right]$ to the $\mathrm{U}(1) \times \mathrm{U}(1)$ model discussed in appendix B by relating the parameters as follows:

$$
\begin{gathered}
\hat{b}=S_{7}, \quad c_{0}=\frac{S_{5}^{2}}{4}, \quad c_{1}=S_{9} S_{1}-\frac{1}{2} S_{5} S_{6}, \\
c_{2}=\frac{1}{4}\left(-4 S_{9} S_{2}+2 S_{5} S_{7}+S_{6}^{2}\right), \quad c_{3}=2 S_{9} S_{3}-\frac{1}{2} S_{6} S_{7} .
\end{gathered}
$$


Because $\left[c_{0}\right],\left[S_{5}\right]$, and $\left[S_{9}\right]$ are trivial, this is simply a redefinition of parameters, not a tuning. Thus, our attempt to obtain the desired U(1) model with the Morrison-Park form has recovered the $\mathrm{U}(1) \times \mathrm{U}(1)$ model, as expected. One can also verify that the standard Morrison-Park section

$$
[\hat{x}: \hat{y}: \hat{z}]=\left[c_{3}^{2}-\frac{2 \hat{b}^{2} c_{2}}{3}:-\frac{1}{2} \hat{b}^{4} c_{1}+\hat{b}^{2} c_{2} c_{3}-c_{3}^{3}: \hat{b}\right]
$$

is given by $\hat{s}_{Q}-\hat{s}_{R}$.

$r=2, q=1$ When $r=2$ and $q=1$ (or equivalently $q=2$ and $r=1$ ), the charged hypermultiplet spectrum takes the form

$$
54 \times( \pm \mathbf{1})+54 \times( \pm \mathbf{2})+54 \times( \pm \mathbf{3}) .
$$

We can try to construct this charge-3 U(1) model with a blown-down version of the $F_{3}$ toric hypersurface construction from [67]. ${ }^{16}$ While there is a Weierstrass model for this construction, it is easier to work with an alternative form of the elliptic fibration where the fiber is a cubic curve in a $\mathbb{P}^{2}$ ambient space with coordinates $[u: v: w]$ :

$$
u\left(s_{1} u^{2}+s_{2} u v+s_{3} v^{2}+s_{5} u w+s_{6} v w+s_{8} w^{2}\right)+v\left(s_{4} v^{2}+s_{7} v w+s_{9} w^{2}\right)=0 .
$$

We take the base of the elliptic fibration to be $\mathbb{P}^{2}$. The charged hypermultiplet spectrum of this construction is

$$
\begin{array}{r}
\left(12 K_{B}^{2}-K_{B}\left(8\left[s_{7}\right]-\left[s_{9}\right]\right)-4\left[s_{7}\right]^{2}+\left[s_{7}\right]\left[s_{9}\right]-\left[s_{9}\right]^{2}\right) \times( \pm \mathbf{1}) \\
+\left(6 K_{B}^{2}-K_{B}\left(4\left[s_{9}\right]-5\left[s_{7}\right]\right)+\left[s_{7}\right]^{2}+2\left[s_{7}\right]\left[s_{9}\right]-2\left[s_{9}\right]^{2}\right) \times( \pm \mathbf{2}) \\
+\left[s_{9}\right] \cdot\left(-K_{B}+\left[s_{9}\right]-\left[s_{7}\right]\right) \times( \pm \mathbf{3}),
\end{array}
$$

and the anomaly coefficient is $\tilde{b}=-6 K_{B}-2\left[s_{7}\right]+4\left[s_{9}\right]$. There are two combinations of $\left(\left[s_{7}\right],\left[s_{9}\right]\right)$ that would seem to give the correct charged hypermultiplet spectrum: $(6 H, 9 H)$ and $(0 H, 6 H)$.

This model is in fact equivalent to the $\mathrm{U}(1) \times \mathrm{U}(1)$ F-theory model for either choice of $\left(\left[s_{7}\right],\left[s_{9}\right]\right)$. If we focus on the $\left(\left[s_{7}\right],\left[s_{9}\right]\right)=(0 H, 6 H)$ possibility, the divisor classes of the other parameters are

$$
\left[s_{1}\right]=3 H, \quad\left[s_{2}\right]=0, \quad\left[s_{3}\right]=-3 H, \quad\left[s_{4}\right]=-6 H, \quad\left[s_{5}\right]=6 H, \quad\left[s_{6}\right]=3 H, \quad\left[s_{8}\right]=9 H .
$$

Because $\left[s_{3}\right]$ and $\left[s_{4}\right]$ are ineffective, $s_{3}$ and $s_{4}$ can be set to 0 , and the elliptic fibration takes the form

$$
u\left(s_{1} u^{2}+s_{2} u v+s_{5} u w+s_{6} v w+s_{8} w^{2}\right)+v w\left(s_{7} v+s_{9} w\right)=0 .
$$

If we then exchange $v \leftrightarrow w$ and identify

$$
\begin{aligned}
& s_{9}=S_{7}, \quad s_{7}=S_{9}, \quad s_{2}=S_{5}, \quad s_{5}=S_{2}, \\
& s_{8}=S_{3}, \quad s_{1}=S_{1}, \quad s_{6}=S_{6},
\end{aligned}
$$

\footnotetext{
${ }^{16}$ Using the more general charge-3 model from [36] does not change the results.
} 
we recover eq. (B.9), which describes the $\mathrm{U}(1) \times \mathrm{U}(1)$ model. Additionally, one can show that the section supporting the $r=2, q=1$ spectrum is given by $2 \hat{s}_{Q}-\hat{s}_{R}$. A similar story holds for $\left(\left[s_{7}\right],\left[s_{9}\right]\right)=(6 H, 9 H)$. We therefore see the expected enhancement to the $\mathrm{U}(1) \times \mathrm{U}(1)$ model for $r=2$ and $q=1$.

$r=3, q=1$ When $r=3$ and $q=1$ (or when $q=3$ and $r=1$ ), the $\mathrm{U}(1)$ charged hypermultiplet spectrum takes the form

$$
54 \times( \pm \mathbf{1})+54 \times( \pm \mathbf{3})+54 \times( \pm 4)
$$

We can attempt to construct this model using the charge-4 U(1) Weierstrass model in [36]. According to the matter spectrum given in [36], we should let $\left[a_{1}\right]$ be $6 H$, [ $\left.b_{1}\right]$ be $9 H$, and $\left[d_{1}\right]$ be $0 H$ to obtain the desired $\mathrm{U}(1)$ spectrum. ${ }^{17}$ The other parameters have the following divisor classes:

$$
\begin{array}{llll}
{\left[d_{0}\right]=-3 H} & {\left[d_{2}\right]=3 H} & {\left[s_{1}\right]=-6 H} & {\left[s_{2}\right]=-3 H} \\
{\left[s_{3}\right]=0 H} & {\left[s_{5}\right]=0 H} & {\left[s_{6}\right]=3 H} & {\left[s_{8}\right]=6 H}
\end{array}
$$

Because their divisor classes are ineffective, the parameters $d_{0}, s_{1}$, and $s_{2}$ should be set to 0 , giving us a Weierstrass model described by

$$
\begin{aligned}
f=-\frac{1}{48}( & \left.s_{6}^{2}-4 s_{3} s_{8}\right)^{2}-\frac{1}{2} s_{5} s_{6} s_{3}\left(a_{1} d_{2}+b_{1} d_{1}\right) \\
& -\frac{1}{3} a_{1}^{2} d_{1}^{2} s_{5}^{2}+\frac{1}{6} a_{1} d_{1} s_{5}\left(s_{6}^{2}+2 s_{3} s_{8}\right)+b_{1} d_{2} s_{5} s_{3}^{2}
\end{aligned}
$$

and

$$
\begin{aligned}
g=\frac{1}{864}( & \left.s_{6}^{2}-4 s_{3} s_{8}\right)^{3}+\frac{1}{4} s_{3}^{2} s_{5}^{2}\left(a_{1}^{2} d_{2}^{2}+b_{1}^{2} d_{1}^{2}\right)-\frac{1}{6} s_{3} s_{6} s_{5}^{2}\left(a_{1} b_{1} d_{1}^{2}+a_{1}^{2} d_{1} d_{2}\right) \\
& +\frac{1}{24} s_{3} s_{6}\left(s_{6}^{2}-4 s_{3} s_{8}\right) s_{5}\left(a_{1} d_{2}+b_{1} d_{1}\right) \\
& -\frac{1}{72} a_{1} d_{1}\left(12 b_{1} d_{2} s_{3}^{2} s_{5}^{2}+\left(s_{6}^{2}-4 s_{3} s_{8}\right)\left(s_{6}^{2}+2 s_{3} s_{8}\right) s_{5}\right) \\
& -\frac{2}{27} a_{1}^{3} d_{1}^{3} s_{5}^{3}+\frac{1}{18} a_{1}^{2} d_{1}^{2}\left(s_{6}^{2}+2 s_{3} s_{8}\right) s_{5}^{2}-\frac{1}{12} b_{1} d_{2} s_{3}^{2}\left(s_{6}^{2}-4 s_{3} s_{8}\right) s_{5} .
\end{aligned}
$$

This Weierstrass model is in fact equivalent to the $\mathrm{U}(1) \times \mathrm{U}(1)$ Weierstrass model of appendix B. To see this, we first note that we can remove all occurrences of $s_{5}$ through the rescalings $d_{2} \rightarrow s_{5}^{-1} d_{2}$ and $d_{1} \rightarrow s_{5}^{-1} d_{1}$; since $\left[s_{5}\right]$ is trivial, the divisions by $s_{5}$ do not cause issues. Then, we can identify the parameters in the two Weierstrass models as follows:

$$
S_{1}=d_{2}, \quad S_{2}=s_{8}, \quad S_{3}=b_{1}, \quad S_{5}=d_{1}, \quad S_{6}=s_{6}, \quad S_{7}=a_{1}, \quad S_{9}=s_{3} .
$$

The Weierstrass models are equivalent, implying that the gauge group is $\mathrm{U}(1) \times \mathrm{U}(1)$ instead of just $\mathrm{U}(1)$. And one can verify that the section that seems to support the $r=3, q=1$ spectrum is in fact equivalent to $3 \hat{s}_{Q}-\hat{s}_{R}$. We therefore see the expected automatic enhancement when we attempt to construct the $r=3, q=1 \mathrm{U}(1)$ model.

\footnotetext{
${ }^{17}$ We could also let $\left[a_{1}\right]=9 H$ and $\left[b_{1}\right]=6 H$, but this situation is equivalent to the $\left[a_{1}\right]=6 H,\left[b_{1}\right]=$ $9 H$ model.
} 
A similar infinite family of $\mathrm{U}(1)$ models with one tensor multiplet was given in [24]. The charged hypermultiplet spectra of these models, which take the form

$$
48 \times( \pm \boldsymbol{r})+48 \times( \pm \boldsymbol{s})+48 \times( \pm(\boldsymbol{r}+\boldsymbol{s}))
$$

mimic the structure of the $T=0$ family discussed above. The analysis in [24] pointed out that there is a related $\mathrm{U}(1) \times \mathrm{U}(1)$ model whose charged hypermultiplet spectrum is

$$
48 \times(\mathbf{1}, \mathbf{0})+48 \times(\mathbf{0}, \mathbf{1})+48 \times(\mathbf{1}, \mathbf{1}) .
$$

As with the $T=0$ infinite family, one can view the $\mathrm{U}(1)$ algebras in the $T=1$ infinite family as linear combinations of the two $\mathrm{U}(1)$ algebras in this $\mathrm{U}(1) \times \mathrm{U}(1)$ model. But we lack the matter necessary to properly Higgs the $\mathrm{U}(1) \times \mathrm{U}(1)$ model down to the desired $\mathrm{U}(1)$ models unless $r, s$, or $r+s$ is 0 . The Automatic Enhancement Conjecture therefore implies that if we attempt to construct the models in this infinite family, the gauge group should enhance to $\mathrm{U}(1) \times \mathrm{U}(1)$. One can confirm that this occurs in the $(r, s)=(1,1),(2,1)$, and $(3,1)$ cases by an explicit analysis of F-theory constructions along the lines above; some aspects of this analysis are discussed in appendix D.

There is another infinite family of U(1) models [25] with zero tensor multiplets and charged hypermultiplet spectra of the form

$$
54 \times( \pm \boldsymbol{a})+54 \times( \pm \boldsymbol{b})+54 \times( \pm \boldsymbol{c})+54 \times( \pm \boldsymbol{d})
$$

where

$$
a=m^{2}-2 m n, \quad b=2 m n-n^{2}, \quad c=m^{2}-n^{2}, \quad d=2\left(m^{2}-m n+n^{2}\right)
$$

for $m, n \in \mathbb{Z}_{+}$with $n \leq \frac{m}{2}$. Some of these models belong to the three-charge infinite family of eq. (5.2), suggesting that the Automatic Enhancement Conjecture may be important for the four-charge family as well. However, we have not yet found an enhanced model for the nontrivial four-charge models. Part of the difficulty lies in the complicated nature of the models: because even the simplest non-trivial members of the family have $\mathrm{U}(1)$ charges larger than those found in the known F-theory constructions, we cannot directly observe any enhancements. And the quadratic structure of the charges, exhibited by their dependence on $m$ and $n$, contrasts with the linear nature of eqs. (5.2) and (5.23) that naturally reflects combinations of multiple $\mathfrak{u}(1)$ algebras. In fact, a similar quadratic structure appears in the $\mathfrak{s u}(13) \oplus \mathfrak{u}(1)$ family of section 5.3. Understanding whether automatic enhancement plays a role in this four-charge family is an important direction for future work.

To summarize, the Automatic Enhancement Conjecture implies that none of the U(1) models in the infinite families of eqs. (5.2) and (5.23) (except for trivial cases with only one type of non-zero charge) can be realized in F-theory, as the gauge group is forced to enhance to $\mathrm{U}(1) \times \mathrm{U}(1)$. The conjecture thus offers a reason as to why those models in the infinite family lie in the F-theory swampland. While finding the "failure mode" for this family is an important step, it does not definitely tell us which $\mathrm{U}(1)$ charges can generally occur in F-theory. Yet this result offers a valuable lesson: limits on the maximum U(1) charge in F-theory likely come not from local constraints on the behavior of sections but from global considerations [18]. The problem with models in the infinite family lies in an enhancement of the Mordell-Weil rank, which is a global property of the fibration. 


\subsection{Infinite family of $\mathfrak{s u}(13) \oplus \mathfrak{u}(1)$ models}

In [24], an infinite family of $T=1$ supergravity models with $\mathfrak{s u}(13) \oplus \mathfrak{u}(1)$ gauge algebras was presented. The charged hypermultiplet spectra for these models take the form

$$
\mathbf{7 8}_{-3 a-\frac{2}{3} f}+3 \times \mathbf{7 8}_{a}+6 \times \mathbf{1 3}_{f}+18 \times \mathbf{1}_{6 a+f},
$$

where

$$
\begin{array}{ll}
a=13 r^{2}-234 r s-51 s^{2} & f=24\left(13 r^{2}+3 s^{2}\right) \\
r=84 n+43 & s=182 n+92
\end{array}
$$

for $n \in \mathbb{Z}$. The anomaly coefficient for the $\mathfrak{u}(1)$ algebra is given by $\tilde{b}=\frac{1}{2}(\alpha, \tilde{\alpha})$ with

$$
\begin{aligned}
& \alpha=52\left[6687 s^{4}+54756 s^{3} r+94458 s^{2} r^{2}-124956 s r^{3}+39455 r^{4}\right], \\
& \tilde{\alpha}=52\left[2475 s^{4}+37908 s^{3} r+170274 s^{2} r^{2}-29484 s r^{3}+9035 r^{4}\right],
\end{aligned}
$$

in a basis where the intersection form is

$$
\Omega=\left(\begin{array}{ll}
0 & 1 \\
1 & 0
\end{array}\right) .
$$

First, note that if we attempt to obtain just the $\mathfrak{s u}(13)$ algebra, the model suffers an automatic enhancement. In order to obtain four antisymmetric and six fundamental hypermultiplets of $\mathfrak{s u}(13)$, the anomaly coefficient $b$ for the $\mathfrak{s u}(13)$ algebra should satisfy $b \cdot b=2$ and $-a \cdot b=4$, implying that $(-6 a-13 b) \cdot b$ is negative and that $-6 a-13 b$ is ineffective. By the discussion in section 4.3, the $\mathfrak{s u}(13)$ algebra should automatically enhance to $\mathfrak{s u}(13) \oplus \mathfrak{u}(1)_{f}$, where we use the subscript $f$ to distinguish this $\mathfrak{u}(1)$ algebra from those in the infinite family. ${ }^{18}$ The charged hypermultiplet spectrum

$$
4 \times \mathbf{7 8}-\frac{1}{13}+6 \times 13_{\frac{6}{13}}
$$

lacks the necessary charged singlets to Higgs away the forced $\mathfrak{u}(1)$ algebra, in line with the Automatic Enhancement Conjecture.

Strictly speaking, this statement only applies to a model with an $\mathfrak{s u}(13)$ gauge algebra, not the $\mathfrak{s u}(13) \oplus \mathfrak{u}(1)$ models in the infinite family. But because we are able to phrase this automatic enhancement locally in terms of $a \cdot b$ and $b \cdot b$, we would expect the $\mathfrak{s u}(13)$ subalgebra of $\mathfrak{s u}(13) \oplus \mathfrak{u}(1)$ to also experience an automatic enhancement. A naive guess is that the gauge algebra for the infinite family automatically enhance to $\mathfrak{s u}(13) \oplus \mathfrak{u}(1) \oplus \mathfrak{u}(1)_{f}$, but if one combines the $\mathfrak{u}(1)$ charges in eq. (5.27) and eq. (5.31), the resulting spectrum does not satisfy the anomaly cancellation conditions. Nevertheless, the expected automatic enhancement of the $\mathfrak{s u}(13)$ algebra suggests models in this $\mathfrak{s u}(13) \oplus \mathfrak{u}(1)$ infinite family should have some inconsistency, even though we have not found an explicit automatic enhancement.

This argument does not seem to capture the true problem with the family, however. There are also seemingly consistent $\mathfrak{s u}(N) \oplus \mathfrak{u}(1)$ infinite families for $N<13$, which can

\footnotetext{
${ }^{18}$ Note that $-3 a-6 b$ is effective, and there should not be automatic enhancements beyond the one to $\mathfrak{s u}(13) \oplus \mathfrak{u}(1)$.
} 
be found by Higgsing the would-be $\mathfrak{s u}(13) \oplus \mathfrak{u}(1)$ models on fundamental matter. The $\mathfrak{s u}(N)$ subalgebras would not suffer the automatic enhancement of $\mathfrak{s u}(13)$ described above, but the mechanism that eliminates the $N<13$ families would presumably apply to the $\mathfrak{s u}(13) \oplus \mathfrak{u}(1)$ family as well. We therefore expect there to be an alternative, more satisfying explanation that rules them out. The quadratic nature of the $\mathfrak{u}(1)$ charges resembles that seen in the four-charge family of eq. (5.25), suggesting that all of these families suffer from similar issues. But it is difficult to see how the quadratic structure of the charges would fit naturally in any kind of linear $\mathfrak{u}(1)$ subalgebra of an enhanced $U(1)^{k}$ theory. Additionally, the charges are significantly larger than those that can be easily realized in F-theory constructions, so it would be tough to directly observe how the models fail. It would be interesting to understand these infinite families more properly in future work.

\subsection{Automatic enhancement for $(\mathrm{SU}(3) \times \mathrm{SU}(2) \times \mathrm{U}(1)) / \mathbb{Z}_{6}$ models}

In [17], the universal Weierstrass model was constructed for the Standard Model gauge group $(\mathrm{SU}(3) \times \mathrm{SU}(2) \times \mathrm{U}(1)) / \mathbb{Z}_{6}$. As discussed there, the model undergoes automatic enhancement for certain choices of the anomaly coefficients $b_{3}, b_{2}, \beta$. In particular, the Jacobian rank method introduced in that paper was used to count the number of moduli for all 98 of the allowed parameter choices for $6 \mathrm{D}$ models over the base $\mathbb{P}^{2}$. In 44 of these cases the moduli count does not match with the expectation from the spectrum fixed by anomaly cancellation, and the model undergoes automatic enhancement or is rendered inconsistent.

In 34 of these cases, the gauge group enhances by the addition of nonabelian or U(1) factors. In each of these cases, the resulting unHiggsed theory is anomaly consistent but has insufficient matter to supersymmetrically Higgs to the original $(\mathrm{SU}(3) \times \mathrm{SU}(2) \times \mathrm{U}(1)) / \mathbb{Z}_{6}$ model, consistent with the Automatic Enhancement Conjecture. In the remaining 10 cases, the discriminant vanishes identically, indicating that the Weierstrass model is invalid. These are all models that would apparently have an unHiggsing to a Tate $\mathrm{SU}(4) \times$ $\mathrm{SU}(3) \times \mathrm{SU}(2)$ model. However, it is found that the corresponding Tate models also have identically vanishing discriminant, corresponding to the stronger case-specific version of the enhancement conjecture that when certain positivity conditions are violated the theory is either enhanced or inconsistent. The various cases are summarized in table 3.

\section{Positivity conditions and local conditions for automatic enhancement}

In each specific class of cases we have encountered in the preceding sections, the pattern is essentially that when we try to tune an F-theory model with algebra $\mathfrak{g}$ with a certain anomaly coefficient $b$ (or $\tilde{b}$ ), and when the curve class associated with a certain other string charge $-n a-m b$ ceases to be effective, then either an additional gauge factor with algebra of at least $\mathfrak{f}$ arises with another anomaly coefficient $b^{\prime}=-p a-q b$, or the theory becomes inconsistent due to excessive singularities. Put more simply, under such circumstances we cannot have a theory with gauge algebra $\mathfrak{g}$ having anomaly coefficient $b$ satisfying the stated conditions unless there is also a gauge algebra component of at least $\mathfrak{f}$ associated with the anomaly coefficient $b^{\prime}$. 


\begin{tabular}{|cccccccccc|}
\hline$\left[b_{1}\right]$ & {$\left[d_{0}\right]$} & {$\left[d_{1}\right]$} & {$\left[d_{2}\right]$} & {$\left[s_{1}\right]$} & {$\left[s_{2}\right]$} & {$\left[s_{5}\right]$} & {$\left[s_{8}\right]$} & $\#$ of Models & Gauge Algebra \\
\hline+ & + & $\geq$ & - & $\geq$ & + & + & $\geq$ & 11 & $\mathfrak{s u}(3) \oplus \mathfrak{s u}(2) \oplus \mathfrak{u}(1) \oplus \mathfrak{u}(1)$ \\
+ & + & - & - & $\geq$ & + & $\geq$ & 0 & 9 & $\mathfrak{s u}(3) \oplus \mathfrak{s u}(3) \oplus \mathfrak{u}(1)$ \\
+ & + & - & - & + & + & + & + & 4 & $\mathfrak{s u}(3) \oplus \mathfrak{s u}(3) \oplus \mathfrak{s u}(2) \oplus \mathfrak{u}(1)$ \\
+ & + & 0 & - & + & + & $\geq$ & - & 3 & $\mathfrak{s u}(4) \oplus \mathfrak{s u}(2) \oplus \mathfrak{u}(1) \oplus \mathfrak{u}(1)$ \\
+ & + & $\geq$ & - & 0 & + & 0 & 0 & 3 & $\mathfrak{s u}(3) \oplus \mathfrak{s u}(2) \oplus \mathfrak{u}(1) \oplus \mathfrak{u}(1) \oplus \mathfrak{u}(1) \oplus \mathfrak{u}(1)$ \\
+ & + & 0 & - & 0 & + & - & - & 1 & $\mathfrak{s u}(5) \oplus \mathfrak{s u}(2) \oplus \mathfrak{u}(1) \oplus \mathfrak{u}(1)$ \\
+ & + & + & - & + & + & 0 & - & 1 & $\mathfrak{s u}(4) \oplus \mathfrak{s u}(2) \oplus \mathfrak{s u}(2) \oplus \mathfrak{u}(1) \oplus \mathfrak{u}(1)$ \\
+ & + & 0 & - & + & + & - & - & 1 & $\mathfrak{s u}(5) \oplus \mathfrak{s u}(2) \oplus \mathfrak{s u}(2) \oplus \mathfrak{u}(1) \oplus \mathfrak{u}(1)$ \\
+ & + & + & - & 0 & + & - & - & 1 & $\mathfrak{s u}(5) \oplus \mathfrak{s u}(3) \oplus \mathfrak{s u}(2) \oplus \mathfrak{u}(1) \oplus \mathfrak{u}(1)$ \\
+ & + & - & - & $\geq$ & + & + & - & 10 & $\operatorname{Invalid}$ \\
\hline
\end{tabular}

Table 3. Cases where the $(\mathrm{SU}(3) \times \mathrm{SU}(2) \times \mathrm{U}(1)) / \mathbb{Z}_{6}$ universal Weierstrass model undergoes automatic enhancement over the base $\mathbb{P}^{2}$. Cases are grouped by their resulting gauge algebra, which is determined by which divisor classes $\left[b_{3}\right]-\left[s_{8}\right]$ associated with parameters in the Weierstrass model are strictly effective $(+)$, effective $(\geq)$, trivial $(0)$, ineffective $(-)$, or unspecified $( \pm)$. The parameter $s_{6}$ is not listed and always has class $-K_{B}=3 H$.

In the context of F-theory, for each such algebra enhancement, the conjecture can thus be stated in terms of the effectiveness of certain curves; in supergravity this translates to conditions that certain anomaly coefficients or other elements of the string charge lattice lie in or outside the positivity cone. While we do not have any completely general way of unifying these positivity conditions into a single conjecture, in each individual case, the statement about positivity conditions is stronger than the Automatic Enhancement Conjecture, since it includes those cases where the theory is rendered invalid by the wouldbe enhancement. In this section we collect the specific results found in the other sections for this stronger condition.

We also collect here some corresponding local versions of the Automatic Enhancement Conjecture. In each case, the general form of the statement is that if there is a gauge component $\mathfrak{g}$ with anomaly coefficient $b$ satisfying certain conditions, then a consistent F-theory model is only possible when there is an additional gauge component of at least $\mathfrak{f}$ associated with another anomaly coefficient $b^{\prime}$. The conditions in the local form for each case only depend on $b \cdot a, b \cdot b$, which correspond to local conditions in the F-theory geometry, namely the genus and self-intersection of the associated curve. These conditions are weaker than the global positivity conditions, but are useful in analyzing models using only part of the structure of the theory.

A number of these conditions that we have encountered in the paper are listed in table 4 . 


\begin{tabular}{|ccccc|}
\hline Algebra $\mathfrak{g}$ & Positivity Condition & Local Condition & $\mathfrak{f}$ & $b^{\prime}$ \\
\hline- & - & $b \cdot b<-2, g=0$ & $G^{\prime} \supseteq \mathrm{SU}(3)$ & $b$ \\
- & - & $b \cdot b \leq-13, g=0$ & no theory & - \\
- & - & $b \cdot b<-2, g>0$ & no theory & - \\
\hline $\mathfrak{s u}(2)$ & $-3 a-b \ngtr 0$ & $b \cdot b>0, b \cdot b>-3 a \cdot b$ & $\mathfrak{s u}(2)$ & $-4 a-b$ \\
$\mathfrak{s u}(2)$ & $b=-3 a$ & - & $\mathfrak{u}(1)$ & $\tilde{b}^{\prime}=-8 a-2 b$ \\
$\mathfrak{s u}(3)$ & $-2 a-b \ngtr 0$ & $b \cdot b>0, b \cdot b>-2 a \cdot b$ & $\mathfrak{s u}(3)$ & $-3 a-b$ \\
$\mathfrak{s u}(3)$ & $b=-2 a$ & - & $\mathfrak{u}(1) \oplus \mathfrak{u}(1)$ & $\tilde{b}^{\prime}=-a\left(\begin{array}{cc}6 & -3 \\
-3 & 6\end{array}\right)$ \\
$\mathfrak{s u}(4)$ & $-3 a-2 b \ngtr 0$ & $b \cdot b>0,2 b \cdot b>-3 a \cdot b$ & $\mathfrak{s u}(2)$ & $-4 a-2 b$ \\
$\mathfrak{s u}(n=20-23)$ & - & $g=0, b \cdot b=1$ & $\mathfrak{s u}(2)$ & $-4 a-\lceil n / 2\rceil b$ \\
$\mathfrak{u}(1)$ & $-6 a-\tilde{b} \ngtr 0$ & $\tilde{b} \cdot \tilde{b}>0, \tilde{b} \cdot \tilde{b}>-6 a \cdot \tilde{b}$ & $\mathfrak{s u}(2)$ & $-4 a-\tilde{b} / 2$ \\
$\mathfrak{u}(1)$ & $\tilde{b}=-6 a$ & - & $\mathfrak{u}(1)$ & $\tilde{b}^{\prime}=-a\left(\begin{array}{cc}2 & -1 \\
-1 & 2\end{array}\right)$ \\
\hline
\end{tabular}

Table 4. Positivity cone and local versions of the Automatic Enhancement Conjecture, for cases with generic matter. In each case a gauge component $\mathfrak{g}$ with anomaly coefficient $b$ satisfying certain conditions forces enhancement to an additional gauge component of at least $\mathfrak{f}$ with anomaly coefficient $b^{\prime}$. (For $\mathfrak{u}(1)$ factors, anomaly coefficients are $\tilde{b}, \tilde{b}^{\prime}$ respectively; note that this anomaly coefficient corresponds to the normalization of the $\mathfrak{u}(1)$ factor so that all charges are integers.) The "genus" contribution $g$ is given in terms of local anomaly coefficients $a, b$ by eq. (3.2). In the "Positivity Condition" column, which gives the conditions on divisor classes under which enhancement occurs, the notation $D \ngtr 0$ means that the divisor class $D$ is ineffective.

Some comments on these local versions of the Automatic Enhancement Conjecture:

- Unlike the general statement of the Automatic Enhancement Conjecture, the local versions of these statements are specific to rather particular circumstances of gauge groups and anomaly coefficients. The list here is not in any way intended to be complete, and even for those cases listed, we have given only some simple sufficient local conditions for each enhancement. It would also be desirable to have a more unified way of framing these in terms of a more general statement analogous to Automatic Enhancement Conjecture.

- In cases where $b^{\prime}$ is not in the positive cone, there is no corresponding F-theory model - the enhancement breaks the theory.

- The local conditions given here are only sufficient conditions, since there can be an enhancement that depends on further structure. In particular, even when the sufficient local conditions are not satisfied, the presence of certain other types of curves intersecting the curve supporting the gauge divisor can provide for an enhancement in more general circumstances. An example of this is given in, for example, sec- 
tion 4.4.2, where we see that the usual enhancement that begins at $\mathfrak{s u}(13)$ on a curve of genus 0 and self-intersection $b \cdot b=2$ also occurs for $\mathfrak{s u}(10-12)$ when the curve in question intersects another curve $b^{\prime}$ of self-intersection $b^{\prime} \cdot b^{\prime}=-1$. A more careful and thorough analysis of local conditions incorporating, e.g., adjacent divisors could be made using similar principles to those used in [43].

- As for the general Automatic Enhancement Conjecture, the positivity based and local versions given here for specific gauge factors are mostly conjectural even at the level of F-theory. The case of non-Higgsable clusters $(G=1)$ was proven in full generality in F-theory in [44], but the other examples depend upon some assumptions on the form of the Weierstrass model used to achieve the initial gauge algebra $G$. In particular, while the general form has been proven for, e.g.,the $\mathfrak{s u}(n)$ algebras under the UFD assumption [42], it is possible that more general forms of the Weierstrass model such as those considered in [28] may evade the conjecture at both the local and global levels. Although such non-UFD constructions in general are expected to give exotic matter representations, this has not been proven to always be the case. Nonetheless, as discussed in appendix D, a variety of effects can remove non-UFD structure, and based on the example presented there it seems likely that these effects conspire to ensure that models without exotic matter take UFD forms.

- As for the general form of the Automatic Enhancement Conjecture, it is natural to speculate that the group-specific stronger versions of the conjecture also hold for $6 \mathrm{D}$ supergravity theories more generally; this possibility is discussed further in the conclusions section.

- We have only listed here cases with generic matter, which depend in general on knowing the corresponding universal Weierstrass models.

- As mentioned earlier, in cases where the automatic enhancement involved would break the theory, such as by producing codimension one $(4,6)$ loci or when $b^{\prime}$ is not in the positive cone, the gauge-specific positivity-based form and the local form of the Automatic Enhancement Conjecture still effectively rule out the relevant swampland models, while the global form as stated in section 2 does not due to the absence of a consistent containing theory. Several examples of this are given in paragraph "Enhancements that break the theory" on page 15 and section 5.3.

- In each case where the local enhancement mechanism forces a gauge group factor with a new anomaly coefficient $b^{\prime}$, as discussed in section 4.2.1, this can also be achieved by having an enhanced gauge factor on each of a set of anomaly coefficients that add to $b^{\prime}=b_{1}+\cdots b_{k}$.

\section{$7 \quad$ Exotic matter and discrete gauge groups}

In this section we discuss several further classes of apparent swampland models, those involving exotic matter and those involving discrete gauge groups. We first describe some 
cases of exotic matter types that should be allowed in F-theory but where certain combinations of these matter representations lie in the apparent swampland. We then briefly describe exotic matter that goes outside the F-theory classification of [67] and situations with discrete gauge groups. These latter two classes of examples are still poorly understood in F-theory, and for these types of apparent swampland models some further work and insights may be needed to fit with the context of the automatic enhancement mechanisms discussed in this paper.

\subsection{An $\mathfrak{s u}(2)$ model with two half-hypermultiplets of 4 matter}

In addition to the fundamental and adjoint representations of $\mathfrak{s u}(2)$, F-theory models can realize matter in the three-index symmetric (4) representation. Such matter is supported at triple point singularities of the divisor supporting the $\mathfrak{s u}(2)$ algebra. More precisely, a triple point singularity of an $\mathfrak{s u}(2)$ divisor in a $6 \mathrm{D}$ F-theory model may support a halfhypermultiplet of $\mathbf{4}$ matter and a full hypermultiplet of fundamental $(\mathbf{2})$ matter $[28,68] .{ }^{19}$ While there are several matter spectra with $\mathbf{4}$ matter that occur in F-theory models, there are also matter spectra that are consistent with the anomaly conditions but pose problems when one tries to construct them in F-theory. One example, described in [28], is a model with no tensor multiplets, an $\mathfrak{s u}(2)$ gauge algebra, and the following spectrum of hypermultiplets:

$$
2 \times \frac{1}{2} \mathbf{4}+84 \times \mathbf{2}+104 \times \mathbf{1}
$$

This spectrum satisfies the anomaly cancellation conditions with $b=5$. We would therefore expect the corresponding F-theory model to have a $\mathbb{P}^{2}$ base, and the $\mathfrak{s u}(2)$ algebra should be tuned on a quintic curve. Because the model supports two half-hypermultiplets of 4 matter, the quintic curve should have two triple points.

But as pointed out in [28], an irreducible quintic curve on $\mathbb{P}^{2}$ cannot have two triple point singularities. If such a quintic existed, we could always find a line that goes through both of the triple points. According to Bezout's theorem, this line should intersect the quintic at five points, counted with multiplicity. The line and the quintic intersect at the two triple points, and each of these intersections would have multiplicity three. Therefore, the triple points alone would give us an intersection number of 6 , which is greater than the expected value of 5 for the total intersection number from Bezout's theorem. This contradiction shows that an irreducible quintic curve cannot have two triple points.

There is a potential loophole in this argument: the quintic curve can contain the line passing through the triple points. In this case, the quintic curve would be reducible. For instance, suppose that, without loss of generality, the two distinct triple points are described by the intersection of a line $\left\{\eta_{b}=0\right\}$ and a quadratic curve $\left\{\eta_{a}=0\right\}$. A quintic curve of the form

$$
\eta_{b}\left(3 t_{(2)} \eta_{a}^{2}+3 t_{(1)} \eta_{a} \eta_{b}+t_{(0)} \eta_{b}^{2}\right)=0
$$

with

$$
\left[t_{(2)}\right]=0 H, \quad\left[t_{(1)}\right]=1 H, \quad\left[t_{(0)}\right]=2 H
$$

\footnotetext{
${ }^{19}$ It is also possible for triple points to support adjoint matter [28], but this possibility is not important for the models considered here.
} 
would then appear to have two triple points. Of course, this quintic curve reduces to the union of a line and a quartic curve. To deform this reducible quintic into an irreducible quintic with two triple points, we would need to introduce a term of the form $t_{(3)} \eta_{a}^{3}$. (All of the terms in the quintic must be polynomials of order three in $\eta_{a}, \eta_{b}$ to preserve the triple point singularities of the quintic and the non-UFD $\mathfrak{s u}(2)$ structure [28].) The parameter $t_{(3)}$ would then be ineffective and would be forced to vanish, giving us the reducible quintic of eq. (7.2) once again. Clearly, there is a geometric obstruction to deforming the reducible quintic into an irreducible curve.

If we were to tune $I_{2}$ singularities along the curve in eq. (7.2), the resulting gauge algebra would be $\mathfrak{s u}(2) \oplus \mathfrak{s u}(2)$ rather than $\mathfrak{s u}(2)$. We take the first $\mathfrak{s u}(2)$ to correspond to the line $\left\{\eta_{b}=0\right\}$ and the second $\mathfrak{s u}(2)$ to correspond to the quartic curve. One can find the corresponding Weierstrass model by setting $t_{(3)}$ to 0 in the $\mathfrak{s u}(2)$ construction given in appendix B of [28]. Each $\left\{\eta_{a}=\eta_{b}=0\right\}$ point supports a half-hypermultiplet of $(\mathbf{2}, \mathbf{3})$ matter and a half-hypermultiplet of $(\mathbf{2}, \mathbf{1})$ matter. The complete hypermultiplet spectrum for this model is

$$
2 \times \frac{1}{2}(\mathbf{2}, \mathbf{3})+1 \times(\mathbf{1}, \mathbf{3})+19 \times(\mathbf{2}, \mathbf{1})+64 \times(\mathbf{1}, \mathbf{2})+104 \times(\mathbf{1}, \mathbf{1}) .
$$

The appearance of the $\mathfrak{s u}(2) \oplus \mathfrak{s u}(2)$ algebra seems linked to the ineffectiveness of the parameter $t_{(3)}$. This is reminiscent of the automatic enhancement examples encountered above, in which the gauge algebra enhances because a parameter becomes ineffective. It is therefore tempting to view the forced reducibility of the quintic curve as a geometric manifestation of the Automatic Enhancement Conjecture. Specifically, we argue that the conjecture implies that any attempt to construct an $\mathfrak{s u}(2)$ model with $b=5$ and two halfhypermultiplets of 4 matter leads to a model with an $\mathfrak{s u}(2) \oplus \mathfrak{s u}(2)$ gauge algebra. In order to show that this is the case, we must examine how one would field-theoretically break the $\mathfrak{s u}(2) \oplus \mathfrak{s u}(2)$ algebra down to $\mathfrak{s u}(2)$.

We are attempting to recombine the 7-branes wrapped on the linear and quartic curves of eq. (7.2) while maintaining the triple points at $\left\{\eta_{a}=\eta_{b}=0\right\}$. Field-theoretically, this recombination process involves giving a VEV to bifundamental matter such that a diagonal $\mathfrak{s u}(2)$ subalgebra of $\mathfrak{s u}(2) \oplus \mathfrak{s u}(2)$ is preserved. Specifically, if the generators for the $\mathfrak{s u}(2)$ on the quartic curve are $T_{(4)}^{a}$ and the generators for the $\mathfrak{s u}(2)$ on the linear curve are $T_{(1)}^{a}$, we wish to preserve the generators

$$
T_{(5)}^{a}=T_{(4)}^{a}+T_{(1)}^{a}
$$

up to some normalization. Of course, the hypermultiplet spectrum in eq. (7.4) does not contain any bifundamental matter, so we cannot perform this Higgsing process. But if we ignore this problem, it naively seems that this Higgsing would give us the $\mathfrak{s u}(2)$ hypermultiplet spectrum in eq. (7.1). The $\mathfrak{s u}(2) \oplus \mathfrak{s u}(2)$ representations in eq. (7.4) branch to representations of the diagonal $\mathfrak{s u}(2)$ as follows:

$$
(2,3) \rightarrow 4+2, \quad(1,3) \rightarrow 3, \quad(2,1) \rightarrow 2, \quad(1,2) \rightarrow 2, \quad(1,1) \rightarrow 1 .
$$

Meanwhile, three of the $\mathfrak{s u}(2) \oplus \mathfrak{s u}(2)$ gauge bosons, those corresponding to the generators

$$
T_{(4)}^{a}-T_{(1)}^{a},
$$


should get masses as part of this Higgsing process. The $T_{(4)}^{a}-T_{(1)}^{a}$ generators appear to be charged in the adjoint $(\mathbf{3})$ representation of the diagonal $\mathfrak{s u}(2)$ algebra, so they must pair up with a hypermultiplet in the $\mathbf{3}$ representation of $\mathfrak{s u}(2)$ to form massive vector multiplets. This 3 hypermultiplet is removed from the massless spectrum. Combining this information with eqs. (7.4) and (7.6) leads to an ostensible massless hypermultiplet spectrum of

$$
2 \times \frac{1}{2} \mathbf{4}+84 \times \mathbf{2}+104 \times \mathbf{1}
$$

in exact agreement with eq. (7.1).

We therefore see that, if we could Higgs the $\mathfrak{s u}(2) \oplus \mathfrak{s u}(2)$ gauge algebra down to $\mathfrak{s u}(2)$, we would recover the expected $\mathfrak{s u}(2)$ model with $b=5$ and two half-hypermultiplets of 4 matter. However, the $\mathfrak{s u}(2) \oplus \mathfrak{s u}(2)$ model in question, with the hypermultiplet spectrum of eq. (7.4), lacks the bifundamental matter necessary to perform this Higgsing. The Automatic Enhancement Conjecture then states that any attempt to construct this $\mathfrak{s u}(2)$ model would lead to a model with an $\mathfrak{s u}(2) \oplus \mathfrak{s u}(2)$ gauge algebra. In other words, an $\mathfrak{s u}(2)$ gauge algebra with the hypermultiplet spectrum of eq. (7.1) would automatically enhance to an $\mathfrak{s u}(2) \oplus \mathfrak{s u}(2)$ gauge algebra. The conjecture thus offers a physical explanation for the geometric observation that a quintic curve with two triple points must be reducible.

The geometric analysis of [28] identified other $\mathfrak{s u}(2)$ models with 4 matter that have similar geometric obstructions. These models would be related to the quintic $\mathfrak{s u}(2)$ model discussed here by applying Cremona transformations to the $\mathbb{P}^{2}$ base of the elliptic fibration. It is natural to expect that these geometric obstructions can also be explained physically by the Automatic Enhancement Conjecture. However, the $\mathfrak{s u}(2)$ divisors in these examples have more complicated algebraic structures, making it more difficult to determine the enhanced gauge algebras. We therefore do not analyze these examples here, although they could serve as interesting tests of the Automatic Enhancement Conjecture in future work.

\subsection{More exotic matter}

We now briefly consider exotic matter in representations not expected from F-theory according to the classification of [28]. Some examples of such matter representations are the 3-index antisymmetric (84) representation of $\mathrm{SU}(9)$, the $\square$ (20) representation of $\mathrm{SU}(4)$, any representation of $\mathrm{G}_{2}$ other than the $\mathbf{7}$ and the adjoint, etc. In general, attempts to construct these representations lead to codimension two $(4,6)$ loci, which are associated with superconformal sectors [50-52]. While there may be a physical interpretation of these SCFT matter structures, these kinds of matter/SCFT structures are as yet poorly understood in F-theory. We may, however, see the appearance of a $(4,6)$ codimension two locus as another kind of "enhancement" that is forced when certain combinations of gauge group and matter fields are tuned in an F-theory model. In particular, this kind of enhancement, or an inconsistency of the theory, seems to arise whenever a theory is tuned in F-theory that could possibly be consistent with exotic matter outside the list of representations identified in [28]. One class of situations in which this $(4,6)$ enhancement has been studied is for the 3-index antisymmetric $\mathrm{SU}(9)$ representation; while there are apparently anomaly-free $6 \mathrm{D}$ supergravity models that contain this representation, any attempt to construct them 
in F-theory appears to lead to a codimension-two $(4,6)$ locus associated with the desired matter representation [13].

Here we briefly analyze another simple set of $6 \mathrm{D}$ supergravity theories with exotic matter that satisfy all known consistency conditions but do not seem to have an F-theory realization. These are theories with gauge group $\mathrm{G}_{2}$, no tensor multiplets, and matter in the 27-dimensional exotic matter representation. Some simple 6D supergravity models with this representation that seem to be anomaly-consistent are, for example, those with $T=0$, gauge group $\mathrm{G}_{2}$, and a matter spectrum of $31 \times \mathbf{7}+0 \times \mathbf{1 4}+x \times \mathbf{2 7}$, where $\mathbf{1 4}$ is the adjoint representation and $x=1$ or $x=2$. We argue here that any attempt to tune such a theory leads to an "enhancement" to a codimension two $(4,6)$ sector.

It is fairly straightforward to see that tuning any kind of $\mathrm{G}_{2}$ gauge factor with matter in the 27 representation leads to some kind of complications in F-theory. As mentioned briefly earlier, one simple way of classifying exotic matter fields is through their contribution to the arithmetic genus of the curve supporting them in an F-theory model, which can be computed directly from the contribution to eq. (3.2) from that particular representation in the anomaly equations [27]. The $\mathbf{2 7}$ of $\mathrm{G}_{2}$ has a genus contribution of $g=3$. Thus, we expect only to see this representation when $\mathrm{G}_{2}$ is tuned on a curve with a triple selfintersection point or other singularity of arithmetic genus 3 . At such a point, however, $(f, g)$ must vanish to orders $(6,9)$ since the orders needed for $\mathrm{G}_{2}$ in the Kodaira classification are $(2,3)$. We thus have an automatic $(4,6)$ point in any F-theory model when we try to tune this representation. The meaning of this kind of singularity is not quite clear at this time. In general, as mentioned above, we expect a superconformal field theory (SCFT) to be coupled in at such codimension $(4,6)$ points. It is possible that such an SCFT could play the role of the $\mathbf{2 7}$ matter, although it is not clear how to make complete sense of this interpretation. It has also been suggested [53] that T-brane structures may somehow enable exotic matter at codimension-two $(4,6)$ loci in such a way that there is no SCFT coupled into the theory, and in principle such a mechanism may operate here, but this is also poorly understood. While the base can be changed by blowing up the $(4,6)$ point associated with the exotic matter, giving another base with $T \rightarrow T+1$, in this case the $\mathrm{G}_{2}$ factor would generally lose its self-intersection at the locus supporting the desired $\mathbf{2 7}$ and the matter associated with that point would be lost. In any case, the enhancement of the Weierstrass model to a codimension two $(4,6)$ point may represent some kind of enhancement related to those described in the bulk of this paper, though it is not covered by the Automatic Enhancement Conjecture as stated in section 2. It would be interesting to try to incorporate this kind of swampland model into a larger framework including the other cases described here that are better understood.

Another interesting class of enhancements related to some kind of strongly coupled SCFT matter arises in the case of a tuned $\mathrm{E}_{8}$ gauge group. ${ }^{20}$ Again here there is a necessary enhancement to a local $(4,6)$ point when we tune, e.g., an $\mathrm{E}_{8}$ factor on a curve on $\mathbb{P}^{2}$, associated with some kind of SCFT. Deformations of the Weierstrass model away from this locus, as for the other tuned $(4,6)$ point SCFTs presumably correspond to some kind

\footnotetext{
${ }^{20}$ Thanks to Yinan Wang for helpful discussions on this point.
} 
of Higgsing that breaks the $\mathrm{E}_{8}$. Some investigation of this kind of structure was carried out in [69], but it would be nice to better understand these kinds of SCFT enhancements and their role in the Automatic Enhancement Conjecture.

\subsection{Discrete gauge groups}

Automatic enhancement can occur for models with discrete gauge symmetries as well, even when the desired charged matter spectrum satisfies the low-energy anomaly conditions of [35]. We do not give a complete description of automatic enhancement for discrete gauge groups here. Instead, we present examples of F-theory models where a $\mathbb{Z}_{2}$ or $\mathbb{Z}_{3}$ symmetry automatically enhances to a larger group, such as $\mathrm{U}(1)$.

For instance, we can consider a $\mathbb{Z}_{2}$ model with zero tensor multiplets and 108 charged hypermultiplets. One might imagine trying to obtain this model by Higgsing a U(1) model with anomaly coefficient $\tilde{b}=6 H$ and 108 hypermultiplets of $( \pm \mathbf{1})$ matter, but this $\mathrm{U}(1)$ model does not have the requisite $( \pm \mathbf{2})$ hypermultiplets to perform the Higgsing. The Automatic Enhancement Conjecture would therefore suggest that the $\mathbb{Z}_{2}$ gauge symmetry should automatically enhance to a $\mathrm{U}(1)$. To see if this enhancement occurs explicitly in F-theory, we can consider the $\mathbb{Z}_{2}$ Weierstrass model from [61, 70], which is given by

$$
y^{2}=x^{3}+\left(e_{1} e_{3}-\frac{1}{3} e_{2}^{2}-4 e_{0} e_{4}\right) x+\left(-e_{0} e_{3}^{2}-\frac{1}{3} e_{1} e_{2} e_{3}-\frac{2}{27} e_{2}^{3}+\frac{8}{3} e_{0} e_{2} e_{4}-e_{1}^{2} e_{4}\right) .
$$

We let the base be $\mathbb{P}^{2}$ to ensure there are zero tensor multiplets, and to obtain the correct number of charged hypermultiplets, we should let $\left[e_{i}\right]=(12-3 i) H \cdot{ }^{21}$ Since $\left[e_{4}\right]$ is trivial, $e_{4}$ can be freely taken to be a perfect square, implying that the gauge group is $\mathrm{U}(1)$ instead of $\mathbb{Z}_{2}$ [61]. We therefore see the expected $\mathbb{Z}_{2} \rightarrow \mathrm{U}(1)$ automatic enhancement.

One can also consider the $F_{1}$ toric hypersurface model discussed in [67], which has gauge group $\mathbb{Z}_{3}$. Considering this model tuned over the base $\mathbb{P}^{2}$, the Jacobian rank method of [17] seems to indicate that this is the universal model for the gauge group $\mathbb{Z}_{3}$. However, for most values of the parameters $\mathcal{S}_{7}, \mathcal{S}_{9}$, the gauge group enhances, due to either the ineffectiveness or triviality of classes of some parameters in the Weierstrass model (in the latter cases, the model develops extra U(1) gauge factors because the associated parameters are automatically perfect squares, as in many of the other cases we have discussed). For example, there is an anomaly-consistent $\mathbb{Z}_{3}$ model with $\left(\mathcal{S}_{7}, \mathcal{S}_{9}\right)=(0,1)$ containing 168 charged hypermultiplets (note that charges \pm 1 and \pm 2 are equivalent in a $6 \mathrm{D} \mathbb{Z}_{3}$ model). However, there is an associated U(1) model (the $F_{3}$ toric hypersurface model of [67]) with $\left(\mathcal{S}_{7}, \mathcal{S}_{9}\right)=(0,1)$ that would Higgs to this $\mathbb{Z}_{3}$ model, except that for this choice of classes the $\mathrm{U}(1)$ model has no $( \pm \mathbf{3})$ matter with which to perform the Higgsing. Thus, according to the Automatic Enhancement Conjecture, we expect that the $\mathbb{Z}_{3}$ model automatically enhances to a $\mathrm{U}(1)$ model for this choice of $\mathcal{S}_{7}, \mathcal{S}_{9}$, and indeed this is the case. It appears to be true that all of the enhanced models over the base $\mathbb{P}^{2}$ have insufficient matter to supersymmetrically Higgs to the associated $\mathbb{Z}_{3}$ model, consistent with the Automatic Enhancement Conjecture. Note that there are also choices of $\mathcal{S}_{7}, \mathcal{S}_{9}$, such as $\left(\mathcal{S}_{7}, \mathcal{S}_{9}\right)=(1,9)$

\footnotetext{
${ }^{21}$ We could alternatively let $\left[e_{i}\right]=3 i H$, but because we can exchange $e_{i} \leftrightarrow e_{4-i}$, the analysis is the same for this case.
} 
that are a priori consistent with anomalies for which the discriminant of the $F_{1}$ model vanishes identically, indicating that these models are actually inconsistent altogether.

It would be interesting to perform a more thorough investigation of automatic enhancements of discrete gauge symmetries in future work.

\section{Automatic enhancement in lower-dimensional theories}

\subsection{Automatic enhancement in $4 \mathrm{D} \mathcal{N}=1$ F-theory models}

While we have focused in this paper on 6D F-theory models, at least at the level of geometry similar enhancements are forced through tuning Weierstrass models with certain gauge groups on sufficiently large divisors in 4D F-theory models as well. Because the actual gauge group can be affected by fluxes and the superpotential, the physical consequences of this geometric enhancement are less clear in $4 \mathrm{D}$ theories, but the analogous phenomenon seems worth exploring.

As a simple example, consider an F-theory model on the base $\mathbb{P}^{3}$, with an $\mathfrak{s u}(2)$ algebra tuned on the divisor $D=b H$, with $H$ the hyperplane divisor. The same logic as that described in section 4.2 applies. Here $K_{B}=-4 H$, and so if $12<b<16$, there will be an additional $\mathfrak{s u}(2)$ algebra arising on the divisor $f_{1}=0$, with $\left[f_{1}\right]=(16-b) H$. As in the $6 \mathrm{D}$ context, the resulting global gauge group, at least at the level of geometry, will be $(\mathrm{SU}(2) \times \mathrm{SU}(2)) / \mathbb{Z}_{2}$.

\subsection{Automatic enhancement in 5D and 4D theories with 8 supercharges}

It is interesting to speculate whether some similar mechanism may arise in $5 \mathrm{D} \mathcal{N}=1$ and $4 \mathrm{D} \mathcal{N}=2$ theories. In particular by compactifying at least some of the cases where $6 \mathrm{D}$ theories have automatic enhancement on circles, it seems we can get some lower-dimensional theories with additional gauge factors that cannot be completely Higgsed away.

As a simple example, consider the $6 \mathrm{D} T=0$ theory with an $\mathfrak{s u}(2)$ tuned on a curve with $b=10$ or $b=11$, which as discussed in section 4.2.1 is automatically enhanced to include an extra $\mathfrak{s u}(2)$ factor on a complementary curve with $\left[f_{1}\right]=2,1$ respectively. If we compactify this $6 \mathrm{D}$ theory down to a $5 \mathrm{D}$ or $4 \mathrm{D}$ theory with 8 supercharges on a circle $S^{1}$ or torus $T^{2}$, the lower-dimensional theory again has a gauge group $(\mathrm{SU}(2) \times \mathrm{SU}(2)) / \mathbb{Z}_{2}$. The vector multiplets now include adjoint scalars for the $\mathfrak{s u}(2)$ factors, so there is a Coulomb branch where, for example, the first $\mathfrak{s u}(2)$ factor remains nonabelian while the second factor is broken to the $\mathrm{U}(1)$ subgroup so that the global group becomes $(\mathrm{SU}(2) \times \mathrm{U}(1)) / \mathbb{Z}_{2}$. In the 5D theory there are no fields charged purely under the $\mathrm{U}(1)$ factor, so it seems that we may have a similar situation where the containing $G^{\prime}$ theory cannot be broken down to a smaller theory with only a gauge algebra $\mathfrak{s u}(2)$ and the same charged matter content under that factor. The story is much less clear here, however, since we do not have an analogous construction to the F-theory geometry where we can see the forcing of the extra factor through the Weierstrass model. If there is some constraint analogous to the automatic enhancement mechanism for 5D theories, for example for 5D models that can be realized by compactifying M-theory on a Calabi-Yau threefold, this would seem to entail 
some constraint on the geometry of general CY threefolds that might hold even beyond the elliptic CY3 framework. We leave this question for further investigation. It would also be interesting to look into the parallel questions for $4 \mathrm{D}$ models coming from compactification on a further circle.

\section{Conclusions and further directions}

In this paper we have identified a range of circumstances in which tuning a certain gauge group $G$ and matter content $M$ in F-theory automatically gives rise to a larger gauge group $G^{\prime}$ and matter content $M^{\prime}$. We have analyzed a variety of constructions for specific gauge groups $G$ that satisfy the conditions of the Automatic Enhancement Conjecture stated in section 2 that there is a containing theory $G^{\prime}, M^{\prime}$ such that the larger theory cannot be broken down to the smaller theory with gauge group $G$ by a supersymmetry-preserving Higgs deformation. For each of the specific gauge groups $G$ with generic matter content, we have used explicit constructions of universal Weierstrass models to identify positivity conditions and associated local conditions in terms of anomaly coefficients that are sufficient to show that certain gauge group and matter combinations cannot be realized in F-theory without some additional enhanced gauge factors. These gauge group-specific formulations of the conjecture are stronger than the general statement since they also incorporate classes of theories that are rendered invalid by the enhancement.

While the analysis here has focused on F-theory constructions, where we can explicitly prove that automatic enhancement must take place at least through standard F-theory constructions, it is interesting to speculate that the Automatic Enhancement Conjecture, and its group-specific stronger versions, may hold more generally for 6D supergravity theories. Indeed, many of the known 6D supergravity models that seem to lie in the swampland [15] of theories that appear consistent but have no known string realization, and which do not violate other known F-theory constraints reviewed in section 3.2.1, are in the class of theories identified here that are automatically enhanced to a larger gauge symmetry when realized in F-theory through standard constructions, or are rendered invalid when this enhancement is not possible. Furthermore, as described in [19] and reviewed in section 4.2.3, another class of apparent swampland theories, in which the massless matter does not span the full charge lattice of the gauge group, appears to be similarly ruled out in F-theory through the appearance of additional sections. It would be interesting to try to find some way of unifying the set of swampland theories that undergo automatic enhancement to either a larger group or an invalid F-theory model, along with those theories that automatically acquire extra Mordell-Weil sections in F-theory, through some condition on the low-energy theory, which would provide a unified swampland hypothesis for all three of these sets of theories in the context of $6 \mathrm{D}$ supergravity. Such a formulation seems a bit subtle, however; for example, in the context of the $\mathfrak{s u}(2) \rightarrow(\mathrm{SU}(2) \times \mathrm{SU}(2)) / \mathbb{Z}_{2}$ described in section 4.2.1, automatic enhancement to the extra $\mathfrak{s u}(2)$ factor is forced on the locus $f_{1}=0$. From the F-theory point of view, this is one particular curve in a moduli space of curves in a given homology class, which is picked out by the structure of the Weierstrass model. In the 6D supergravity theory, that homology class corresponds to a particular charge in the string 
charge lattice, but the degrees of freedom associated with the specific choice of curve $f_{1}$ are not clearly visible in the low-energy theory in any way that we are familiar with; thus, in particular, world-volume methods on a string such as those used in $[2,41]$ do not seem sensitive to the specific locus $f_{1}=0$ as distinct from the other string configurations coming from branes wrapped on other cycles in the same class. Understanding this better would be an interesting direction for further investigation.

Another question of interest is whether the Automatic Enhancement Conjecture can be rigorously proven even in the context of F-theory. For the first case we have discussed, that of non-Higgsable clusters, such a proof exists. In this situation, where there is a curve $C$ of negative self-intersection $C \cdot C \leq-3$, the curve $C$ is rigid and hence unique, and the arguments of [44] show that any F-theory model with such a curve must have a non-Higgsable gauge group of at least SU(3). For the other cases we have described here, however, the argument is not quite so complete. We have shown that standard Ftheory constructions with various gauge groups and matter content $G, M$ are automatically enhanced to $G^{\prime}, M^{\prime}$, however, and while we have not ruled out the possibility of exotic Ftheory constructions such as those using non-UFD structures on singular curves [28] that could in principle realize $G, M$ theories without enhancement, the results of appendix D suggest that this approach will not lead to theories that violate the conjecture. Another way to prove the Automatic Enhancement Conjecture for many of the classes of examples we have described here would be to show that the universal moduli space of theories with at least a given gauge group and matter content $G, M$ must be a connected set (within the context of a class of theories with a given F-theory base $B$, corresponding to a fixed string charge lattice and positivity cone in the low-energy theory, and assuming fixed anomaly coefficients for the factors of $G$ ). If this is true, then every theory with $G, M$ could be reached from every other, so that the enhanced theories $G^{\prime}, M^{\prime}$ would need to be connected to any smaller theory such as one with only the gauge group and matter content $G, M$ and no enhancement, which is not allowed by the conditions of the Automatic Enhancement Conjecture. If such an argument could be realized, one could imagine a similar argument might apply in the more general context of $6 \mathrm{D}$ supergravity without reference to an F-theory completion, if it were possible to show that for a given string charge lattice and positivity cone at a fixed number of tensor multiplets $T$ the moduli space of allowed supergravity theories with at least a given $G, M$ is connected, for fixed values of the anomaly coefficients.

\section{Acknowledgments}

We would like to thank Lara Anderson, James Gray, Daniel Harlow, Sheldon Katz, David Morrison, Paul Oehlmann, Houri Tarazi, Cumrun Vafa, and Yinan Wang for helpful discussions. The work of APT and WT was supported by DOE grant DE-SC00012567. APT is supported in part by the DOE (HEP) Award DE-SC0013528. NR is supported by NSF grant PHY-1720321. 


\section{A Explicit analysis of rational sections}

\section{A.1 $(\mathrm{SU}(2) \times \mathrm{U}(1)) / \mathbb{Z}_{2}$ model}

For the $\mathfrak{s u}(2) \rightarrow(\mathrm{SU}(2) \times \mathrm{U}(1)) / \mathbb{Z}_{2}$ enhancement of section 4.2 .2 , we take the Weierstrass model in eq. (4.1) and consider situations where the divisor class $\left[g_{2}\right]$ is trivial. The parameter $g_{2}$ is then essentially a constant, and we can freely set it to a perfect square $\gamma^{2}$ without issue, where $[\gamma]$ is trivial. This is not a tuning of the model when $\left[g_{2}\right]=0$ but is instead a simple rewriting of $g_{2}$. After this substitution, the Weierstrass model is described by

$$
f=-\frac{1}{48} \phi^{2}+\sigma f_{1}, \quad g=\frac{1}{864} \phi^{3}-\frac{1}{12} \phi \sigma f_{1}+\sigma^{2} \gamma^{2} .
$$

This Weierstrass model is in fact in the Morrison-Park form $[18]^{22}$

$$
f=c_{1} c_{3}-\hat{b}^{2} c_{0}-\frac{1}{3} c_{2}^{2}, \quad g=c_{0} c_{3}^{2}-\frac{1}{3} c_{1} c_{2} c_{3}+\frac{2}{27} c_{2}^{3}-\frac{2}{3} \hat{b}^{2} c_{0} c_{2}
$$

with

$$
\hat{b}=1, \quad c_{0}=\frac{\phi^{2}}{64}-f_{1} \sigma, \quad c_{1}=-2 \gamma \sigma, \quad c_{2}=-\frac{1}{8} \phi, \quad c_{3}=0 .
$$

It therefore admits an infinite-order generating section

$$
[\hat{x}: \hat{y}: \hat{z}]=\left[\frac{\phi}{12}: \gamma \sigma: 1\right]
$$

and the gauge algebra is $\mathfrak{s u}(2) \oplus \mathfrak{u}(1)$. Because $[\gamma]$ is trivial, the only $\mathfrak{u}(1)$-charged matter occurs at $\left\{\sigma=f_{1}^{2}-\gamma^{2} \phi=0\right\}$; this locus supports $\mathbf{2}_{1 / 2}$ matter, as can be verified with the techniques in [56]. ${ }^{23}$ This matter spectrum satisfies the anomaly cancellation conditions for $\tilde{b}=-2 K_{B}-\frac{1}{2}[\sigma]$, and the gauge group is $(\mathrm{SU}(2) \times \mathrm{U}(1)) / \mathbb{Z}_{2}$.

\section{A.2 $(\mathrm{SU}(2) \times \mathrm{SU}(2)) / \mathbb{Z}_{2}$ and $\mathrm{SU}(2) / \mathbb{Z}_{2}$ models}

For the situations encountered in sections 4.2.1 and 4.2.3, we take the Weierstrass model given by eq. (4.1) and consider situations where the divisor class $\left[g_{2}\right]$ is ineffective. The parameter $g_{2}$ is therefore 0 , leading to a Weierstrass model with

$$
f=-\frac{1}{48} \phi^{2}+\sigma f_{1}, \quad g=\frac{1}{864} \phi^{3}-\frac{1}{12} \phi \sigma f_{1} .
$$

This Weierstrass model admits a rational section of the form

$$
[\hat{x}: \hat{y}: \hat{z}]=\left[\frac{\phi}{12}: 0: 1\right] \text {, }
$$

which is a torsional section of order 2 . The section is directly correlated with the $\mathbb{Z}_{2}$ quotient seen in the gauge groups [19]. In fact, this torsional section can be viewed as the $\gamma \rightarrow 0$ enhancement of the rational section in eq. (A.4) along the lines of [57].

\footnotetext{
${ }^{22}$ To avoid confusion with the anomaly coefficients, we use the symbol $\hat{b}$ to denote the parameter typically called $b$.

${ }^{23}$ The $\mathfrak{u}(1)$ charges are normalized here such that the lattice of singlet charges has unit spacing [71]. While there are no charged singlets when $[\gamma]=0$, there are charged singlets at $\left\{f_{1}=\gamma=0\right\}$ for other choices of $[\gamma]$. The charge of these would-be singlets sets the normalization.
} 


\section{B $\quad \mathrm{U}(1) \times \mathrm{U}(1)$ model}

In section 5, we make use of a $\mathrm{U}(1) \times \mathrm{U}(1)$ F-theory model based on the construction in [72]. (A more general class of F-theory models with group $\mathrm{U}(1) \times \mathrm{U}(1)$ was constructed in [73], but the more general form is not needed here.) The base of the elliptic fibration is $\mathbb{P}^{2}$, such that the resulting $6 \mathrm{D}$ spectrum has zero tensor multiplets. We take the divisor classes of the parameters to be the following: ${ }^{24}$

$$
\begin{array}{llll}
{\left[S_{1}\right]=3 H,} & {\left[S_{2}\right]=6 H,} & {\left[S_{3}\right]=9 H,} & {\left[S_{5}\right]=0 H,} \\
{\left[S_{6}\right]=3 H,} & {\left[S_{7}\right]=6 H,} & {\left[S_{8}\right]=-3 H,} & {\left[S_{9}\right]=0 H .}
\end{array}
$$

The Weierstrass construction for this $\mathrm{U}(1) \times \mathrm{U}(1)$ model, with $S_{8}$ set to 0 due to its ineffective divisor class, is described by

$$
\begin{aligned}
f=-\frac{1}{48} & \left(S_{6}^{2}-4 S_{5} S_{7}\right)^{2} \\
& +\frac{1}{6} S_{9}\left(6 S_{1} S_{3} S_{9}-3 S_{1} S_{6} S_{7}-2 S_{2}^{2} S_{9}+2 S_{2} S_{5} S_{7}+S_{2} S_{6}^{2}-3 S_{3} S_{5} S_{6}\right)
\end{aligned}
$$

and

$$
\begin{gathered}
g=\frac{1}{864}\left(S_{6}^{2}-4 S_{5} S_{7}\right)^{3}+\frac{1}{27} S_{2} S_{9}^{3}\left(9 S_{1} S_{3}-2 S_{2}^{2}\right) \\
-\frac{1}{72} S_{9}\left(S_{6}^{2}-4 S_{5} S_{7}\right)\left(-3 S_{1} S_{6} S_{7}+S_{2}\left(2 S_{5} S_{7}+S_{6}^{2}\right)-3 S_{3} S_{5} S_{6}\right) \\
+\frac{1}{36} S_{9}^{2}\left[9 S_{1}^{2} S_{7}^{2}-3 S_{3}\left(S_{1}\left(2 S_{5} S_{7}+S_{6}^{2}\right)+2 S_{2} S_{5} S_{6}\right)\right. \\
\left.\quad-6 S_{1} S_{2} S_{6} S_{7}+2 S_{2}^{2}\left(2 S_{5} S_{7}+S_{6}^{2}\right)+9 S_{3}^{2} S_{5}^{2}\right]
\end{gathered}
$$

The discriminant is then proportional to $S_{9}^{2}$, but because $\left[S_{9}\right]$ is trivial, this factor does not signal the appearance of a nonabelian gauge algebra. There are two generating sections for this elliptic fibration. The first, $\hat{s}_{Q}$, is given by

$$
\left[\hat{x}_{Q}: \hat{y}_{Q}: \hat{z}_{Q}\right]=\left[\frac{1}{12}\left(S_{6}^{2}-4\left(S_{2} S_{9}+S_{5} S_{7}\right)\right):-\frac{1}{2} S_{9}\left(S_{3} S_{5}-S_{1} S_{7}\right): 1\right]
$$

The second, $\hat{s}_{R}$, is given by

$$
\left[\hat{x}_{R}: \hat{y}_{R}: \hat{z}_{R}\right]=\left[\frac{1}{12}\left(S_{6}^{2}-4 S_{2} S_{9}+8 S_{5} S_{7}\right): \frac{1}{2}\left(S_{5} S_{6} S_{7}-S_{9}\left(S_{1} S_{7}+S_{3} S_{5}\right)\right): 1\right] .
$$

The presence of these two independent sections implies that the gauge group is $\mathrm{U}(1) \times \mathrm{U}(1)$.

According to the original matter analysis in [72], the locus $\left\{S_{8}=S_{9}=0\right\}$ supports $(\mathbf{- 1}, \mathbf{- 2})$ matter, and the locus $\left\{S_{7}=S_{9}=0\right\}$ supports $(\mathbf{0}, \mathbf{2})$ matter. Because $\left[S_{9}\right]$ is trivial, the model does not support any hypermultiplets of $(\mathbf{- 1 , - 2 )}$ or $(\mathbf{0}, \mathbf{2})$ matter. There is also $(\mathbf{1}, \mathbf{1})$ matter at

$$
\left\{S_{7} S_{8}^{2}-S_{6} S_{8} S_{9}+S_{5} S_{9}^{2}=S_{3} S_{8}^{2}-S_{2} S_{8} S_{9}+S_{1} S_{9}^{2}=0\right\}
$$

\footnotetext{
${ }^{24}$ Even though the variables are written as $s_{i}$ in [72], we write them here as $S_{i}$ to avoid confusion with variables used elsewhere in this paper.
} 
in the original model. The class of $S_{7} S_{8}^{2}-S_{6} S_{8} S_{9}+S_{5} S_{9}^{2}$ is trivial in this example, and there are no hypermultiplets of $(\mathbf{1}, \mathbf{1})$ matter. However, $(\mathbf{1}, \mathbf{- 1})$ matter is supported at $\left\{S_{3}=S_{7}=0\right\}$, implying that this model has 54 hypermultiplets of $(\mathbf{1}, \mathbf{- 1})$ matter. By examining the loci where $\hat{y}$ and $3 \hat{x}^{2}+f \hat{z}^{4}$ for the two sections simultaneously vanish, one can verify that there are 54 hypermultiplets of $(\mathbf{1 , 0 )}$ matter and 54 hypermultiplets of $(\mathbf{0}, \mathbf{1})$ matter. In summary, the total spectrum is

$$
54 \times(\mathbf{1}, \mathbf{- 1})+54 \times(\mathbf{1}, \mathbf{0})+54 \times(\mathbf{0}, \mathbf{1}) .
$$

The anomaly coefficient for the model is given by

$$
\tilde{b}=\left(\begin{array}{cc}
6 H & -3 H \\
-3 H & 6 H
\end{array}\right)
$$

It is also useful to write this model in an alternative form where the fiber is a cubic curve in a $\mathbb{P}^{2}$ ambient space. If the coordinates for this $\mathbb{P}^{2}$ ambient space are $[u: v: w]$, the model is described by

$$
u\left(S_{1} u^{2}+S_{2} u v+S_{3} v^{2}+S_{5} u w+S_{6} v w\right)+v w\left(S_{7} v+S_{9} w\right)=0 .
$$

The zero section is given by $\{u=v=0\}$, while the two generating sections are given by $\{u=w=0\}$ and $\left\{u=S_{7} v+S_{9} w=0\right\}$.

\section{Absence of adjoint matter on $C$ when $C+K_{B}$ is not effective}

In various circumstances we consider nonabelian gauge factors with anomaly coefficient $b$ such that $b+a$ is outside the positivity cone. In such circumstances we assert that in any F-theory realization the nonabelian gauge factor in question cannot have adjoint matter. For example, this comes up in section 4.2.1, where $\left[g_{2}\right]=-4 K_{B}-C$ is not in the cone of effective curves, but $C^{\prime}=\left[f_{1}\right]=-3 K_{B}-C$ is effective, when an $\mathfrak{s u}(2)$ algebra is tuned on a curve of class $C$, leading to an enhancement with at least an $\mathfrak{s u}(2)$ on a curve of class $C^{\prime}$. In this appendix we give a simple F-theory based physics argument for why a nonabelian gauge factor on a curve of class $C^{\prime}$ cannot have adjoint matter when $C^{\prime}+K_{B}$ is not effective, and we summarize a simple argument for this conclusion from algebraic geometry. We also illustrate how this works explicitly in a general class of situations at $T \leq 9$, which highlights the subtleties that arise in the classification of F-theory constructions when $T \geq 9$.

First, we give a simple physics argument based on the Morrison-Park model (A.2). Given a curve class $C^{\prime}=\left[c_{3}\right]$, we can perform a Tate tuning of an $\mathfrak{s u}(2)$ algebra over $c_{3}$, which essentially gives the Morrison-Park model with $\hat{b}=0$. When $[\hat{b}]=C^{\prime}+K_{B}$ is effective, we can deform the $\mathfrak{s u}(2)$ model to the general Morrison-Park form by turning on a nonzero value for $\hat{b}$. This corresponds physically to breaking $\mathfrak{s u}(2) \rightarrow \mathfrak{u}(1)$ by Higgsing on an adjoint field. When $[\hat{b}]$ is not effective there is no such deformation, and so no adjoint fields can be present in the original $\mathfrak{s u}(2)$ theory. Geometrically, this means that the curve $C^{\prime}$ is a rational curve, of genus zero. 
This conclusion also follows from some basic considerations of algebraic geometry. ${ }^{25}$ We assume that $C^{\prime}$ is an irreducible effective curve of (arithmetic) genus $g=1+C^{\prime}$. $\left(C^{\prime}+K_{B}\right) / 2>0$ in a complex surface $B$ that supports an elliptic Calabi-Yau threefold of interest for F-theory. We furthermore assume that $B$ is rational; this leaves out the case of the Enriques surface, which is essentially trivial for F-theory. The Riemann-Roch theorem for surfaces states that

$$
\chi\left(\mathcal{O}\left(C^{\prime}+K_{B}\right)\right)=C^{\prime} \cdot\left(C^{\prime}+K_{B}\right) / 2+\chi\left(\mathcal{O}_{B}\right),
$$

where $\chi\left(\mathcal{O}_{B}\right)=1$ since $B$ is rational, and $\mathcal{O}\left(C^{\prime}+K_{B}\right)$ is the line bundle associated with $C^{\prime}+K_{B}$. We thus have

$$
\chi\left(\mathcal{O}\left(C^{\prime}+K_{B}\right)\right)=h^{2}\left(\mathcal{O}\left(C^{\prime}+K_{B}\right)\right)-h^{1}\left(\mathcal{O}\left(C^{\prime}+K_{B}\right)\right)+h^{0}\left(\mathcal{O}\left(C^{\prime}+K_{B}\right)\right)=g .
$$

Serre duality tells us that

$$
h^{2}\left(\mathcal{O}\left(C^{\prime}+K_{B}\right)\right)=h^{0}\left(\mathcal{O}\left(-C^{\prime}\right)\right),
$$

but $h^{0}\left(\mathcal{O}\left(-C^{\prime}\right)\right)=0$ since $-C^{\prime}$ is not effective. It follows that

$$
h^{0}\left(\mathcal{O}\left(C^{\prime}+K_{B}\right)\right) \geq g>0,
$$

so the line bundle $\mathcal{O}\left(C^{\prime}+K_{B}\right)$ has nontrivial sections and thus $C^{\prime}+K_{B}$ is effective under the assumptions made above. This implies, on the other hand, that if $C^{\prime}+K_{B}$ is not effective but $C^{\prime}$ is irreducible and effective, then $C^{\prime}$ has genus $g=0$, which is what we set out to prove.

To illustrate this result more explicitly, we demonstrate the situation for $T \leq 9$ and curves of limited complexity (e.g., for curves of limited degree on blow-ups of $\mathbb{P}^{2}$ ), and briefly described the complications that arise at larger values of $T$ and for curves of higher complexity. To proceed explicitly we consider the class of cases where there is a basis of curves with intersection form $(+1,-1,-1, \ldots,-1)$ and we can write $K_{B}=(3,-1,-1, \ldots,-1)$. Since all bases can be found as multiple blowups of $\mathbb{P}^{2}$ or the Hirzebruch surfaces $\mathbb{F}_{m}, m \leq 12$ [8], this covers all cases except the Hirzebruch surfaces with even $m$, which can be treated with a similar but simpler argument. For the del Pezzo surfaces $d P_{k}$ given by blowing up $k<9$ points in general position on $\mathbb{P}^{2}$, a basis for the cone of effective divisor classes is given by $(1,0, \ldots, 0)$, corresponding to a line on the original $\mathbb{P}^{2}$ that does not pass through any of the blown up points, $(0, \ldots, 0,1,0, \ldots, 0)$, corresponding to the exceptional curve of the $i$ th blown up point, $(1,0, \ldots, 0,-1,0, \ldots, 0,-1,0, \ldots, 0)$ where the -1 entries appear in positions $i, j$, corresponding to a line passing through the corresponding two blowup points, $(2, \ldots)$ with up to $5-1$ entries (and the remaining entries being 0 ), corresponding to a conic passing through up to 5 of the blowup points, $(3, \ldots)$ with up to $k<9-1$ entries (or one -2 and up to seven $-1 \mathrm{~s}$ ), corresponding to irreducible cubics, etc.. For curves of degree $n>3$, the set of possibilities can be complicated by constraints on the possible multiplicities of singularities (see, e.g., [28]). When the points are not in general position,

\footnotetext{
${ }^{25}$ Thanks to Sheldon Katz for this proof.
} 
the effective cone is slightly larger and includes curves of self-intersection $C \cdot C<-1$, corresponding to curves of degree $d$ in the original $\mathbb{P}^{2}$ that pass through more than $d^{2}+1$ points (possibly including multiplicities).

If a curve $C^{\prime}$ is irreducible, we have $\left(K_{B}+C^{\prime}\right) \cdot C^{\prime}=2 g-2$, so the condition that $g>0$ is that $\left(K_{B}+C^{\prime}\right) \cdot C^{\prime} \geq 0$. Writing $C^{\prime}=\left(n,-m_{1},-m_{2}, \ldots,-m_{T}\right)$ in the given basis, this condition becomes

$$
n(n-3) \geq \sum_{i} m_{i}\left(m_{i}-1\right) .
$$

For $n=3$, the only solutions to this condition have all $m_{i}=0,1$, and so

$$
C^{\prime}+K_{B}=\left(n-3,1-m_{1}, \ldots, 1, m_{T}\right)=(0,0 \text { or } 1, \ldots, 0 \text { or } 1)
$$

is clearly in the effective cone. For $n=4$, we can have up to two values of $m_{i}=2$. But we then have, e.g., the worst case scenario (largest $m_{i} \mathrm{~s}$ )

$$
C^{\prime}+K_{B}=(1,-1,-1,0, \ldots, 0)
$$

which again lies in the effective cone. For $n=5$, we can have $m_{i}$ s above 1 in the combinations $(3,2,2)$ or $(2,2,2,2,2)$. These give (with all other $m$ s equal to 1 )

$$
C^{\prime}+K_{B}=(2,-2,-1,-1,0, \ldots, 0),(2,-1,-1,-1,-1,-1,0, \ldots, 0),
$$

which again are easily seen to be in the effective cone.

A similar argument holds for larger values of $n$, as long as $T \leq 9$, though as $n$ becomes increasingly large the set of effective curves becomes more subtle; for example, $C^{\prime}+K_{B}=$ $\left(3,-2,-1^{7}\right)$ saturates eq. (C.5), and depends on the fact that a cubic can be found on $\mathbb{P}^{2}$ with a double point at one chosen point and passing through seven other arbitrary points. When $T \geq 9$, however, we can also see more subtle issues arising related to the choice of which bases support an elliptic fibration, as well as what kinds of singularities are possible on a curve of given degree. For example, at $T=9, n=6$, we can choose $C^{\prime}=(6,-2, \ldots,-2)$. We then have $C^{\prime}+K_{B}=(3,-1, \ldots,-1)=-K_{B}$. We cannot, however, blow up $\mathbb{P}^{2}$ at 9 generic points and get an acceptable F-theory base; the base that results from blowing up at 9 generic points has a rigid cubic in the class $K_{B}$, so the Weierstrass model has $f, g$ that generically vanish to orders $(4,6)$ on $K_{B}$. For $T=9$, this still clearly works as $K_{B}=(3,-1, \ldots,-1)$ is effective (and non-rigid) on any acceptable F-theory base. The situation, however, clearly becomes more complicated for larger $T$, since choosing curves of the form $C^{\prime}=(6, \ldots)$ gives classes $C^{\prime}+K_{B}=(3, \ldots)$ with $9-1$ entries, curves of the form $C^{\prime}=(7, \ldots)$ can have $m$ s of, e.g., $14 \times 2$, etc.. Some of the complications that arise in determining the generators of the effective cone at larger $T$ are discussed further in, e.g., [74]. An argument of this form must in principle work for all $T$ and all allowed F-theory bases, since the more abstract mathematical argument above makes the statement rigorous at all $T$. 


\section{Another test of automatic enhancement in a U(1) model}

In this appendix, we perform an additional test of automatic enhancement for the $r=s=1$ model in the $T=1$ infinite family of eq. (5.23). Recall that the charged hypermultiplet spectrum for this model is

$$
96 \times( \pm \mathbf{1})+48 \times( \pm \mathbf{2}),
$$

and we expect an automatic enhancement to a $\mathrm{U}(1) \times \mathrm{U}(1)$ model with a charged hypermultiplet spectrum of the form

$$
48 \times(\mathbf{1}, \mathbf{0})+48 \times(\mathbf{0}, \mathbf{1})+48 \times(\mathbf{1}, \mathbf{1}) .
$$

Because there is one tensor multiplet, the base of an F-theory model realizing this spectrum would be $\mathbb{F}_{n}$. If we attempt to construct this U(1) model using the Morrison-Park tuning of eq. (A.2) with an $\mathbb{F}_{n}$ base, the parameters should have the following divisor classes:

$$
[\hat{b}]=-2 K_{B}, \quad\left[c_{m}\right]=-m K_{B} .
$$

Because $\left[c_{0}\right]$ is trivial, we see an enhancement to $\mathrm{U}(1) \times \mathrm{U}(1)$ as discussed in section 5.2.

One might imagine circumventing this enhancement by obtaining the $\mathrm{U}(1)$ model from constructions that typically give higher charges. ${ }^{26}$ Let us focus on the charge- 3 construction from [36], which supports charge-3 matter at $\left\{\eta_{a}=\eta_{b}=0\right\}$. It is known that the construction is equivalent to the Morrison-Park form if either $\left[\eta_{a}\right]$ or $\left[\eta_{b}\right]$ is trivial. But if we choose non-trivial $\left[\eta_{a}\right]$ and $\left[\eta_{b}\right]$ such that $\left[\eta_{a}\right] \cdot\left[\eta_{b}\right]=0$, the model would only support hypermultiplets with charges \pm 1 and \pm 2 . This model does not seem to be in Morrison-Park form, and one might hope it would realize eq. (D.1) without an automatic enhancement.

However, various effects either render the model invalid or secretly put it into MorrisonPark form. The charge- 3 construction contains a parameter $\bar{\phi}$ that is important for its structure. If $\bar{\phi}, \eta_{a}$, and $\eta_{b}$ share a common nontrivial factor, $(f, g, \Delta)$ vanish to orders $(4,6,12)$ at a codimension-one locus. Alternatively, if $\bar{\phi}$ can be written as

$$
\phi_{a} \eta_{a}+\phi_{b} \eta_{b}
$$

we can relate the charge-3 construction to the Morrison-Park form through the following map of parameters: ${ }^{27}$

$$
\begin{aligned}
\hat{b}= & b_{(2)} \eta_{a}^{2}+2 b_{(1)} \eta_{a} \eta_{b}+b_{(0)} \eta_{b}^{2}, \\
c_{3}= & -\left(t_{(0)} \eta_{b}^{3}+3 t_{(1)} \eta_{a} \eta_{b}^{2}+3 t_{(2)} \eta_{a}^{2} \eta_{b}+t_{(3)} \eta_{a}^{3}\right) \\
& +\frac{1}{12}\left(b_{(2)} \eta_{a}^{2}+2 b_{(1)} \eta_{a} \eta_{b}+b_{(0)} \eta_{b}^{2}\right)\left(b_{(0)} \eta_{b} \phi_{a}+b_{(1)} \eta_{a} \phi_{a}-b_{(1)} \eta_{b} \phi_{b}-b_{(2)} \eta_{a} \phi_{b}\right), \\
c_{2}= & \frac{1}{4}\left(h_{(2)} \eta_{a}^{2}+2 h_{(1)} \eta_{a} \eta_{b}+h_{(0)} \eta_{b}^{2}\right)+\frac{1}{144}\left(b_{(1)}^{2}-b_{(0)} b_{(2)}\right)\left(\phi_{a} \eta_{a}+\phi_{b} \eta_{b}\right)^{2} \\
& +\frac{1}{96}\left(b_{(2)} \phi_{b}^{2}-2 b_{(1)} \phi_{a} \phi_{b}+b_{(0)} \phi_{a}^{2}\right)\left(b_{(2)} \eta_{a}^{2}+2 b_{(1)} \eta_{a} \eta_{b}+b_{(0)} \eta_{b}^{2}\right) \\
& +\frac{1}{4}\left(t_{(1)} \eta_{b}^{2}+2 t_{(2)} \eta_{a} \eta_{b}+t_{(3)} \eta_{a}^{2}\right) \phi_{b}-\frac{1}{4}\left(t_{(0)} \eta_{b}^{2}+2 t_{(1)} \eta_{a} \eta_{b}+t_{(2)} \eta_{a}^{2}\right) \phi_{a},
\end{aligned}
$$

\footnotetext{
${ }^{26}$ The authors thank Yinan Wang for helpful discussions on this point.

${ }^{27}$ This can be understood through the normalized intrinsic structure $[28,36]$ of the charge- 3 construction.
} 


$$
\begin{aligned}
c_{1}= & \frac{1}{1728}\left(b_{(2)} \phi_{b}^{2}-2 b_{(1)} \phi_{a} \phi_{b}+b_{(0)} \phi_{a}^{2}\right)\left(b_{(0)} \eta_{b} \phi_{a}+b_{(1)} \eta_{a} \phi_{a}-b_{(1)} \eta_{b} \phi_{b}-b_{(2)} \eta_{a} \phi_{b}\right) \\
& -\frac{\eta_{a}}{48}\left(48 \lambda_{(1)}-2 h_{(1)} \phi_{a}+2 h_{(2)} \phi_{b}+t_{(1)} \phi_{a}^{2}-2 t_{(2)} \phi_{a} \phi_{b}+t_{(3)} \phi_{b}^{2}\right) \\
& -\frac{\eta_{b}}{48}\left(48 \lambda_{(0)}-2 h_{(0)} \phi_{a}+2 h_{(1)} \phi_{b}+t_{(0)} \phi_{a}^{2}-2 t_{(1)} \phi_{a} \phi_{b}+t_{(2)} \phi_{b}^{2}\right) \\
c_{0}= & -f_{2}+\frac{1}{82944}\left(b_{(2)} \phi_{b}^{2}-2 b_{(1)} \phi_{a} \phi_{b}+b_{(0)} \phi_{a}^{2}\right)^{2} \\
& +\frac{1}{576}\left(h_{(0)} \phi_{a}^{2}-2 h_{(1)} \phi_{a} \phi_{b}+h_{(2)} \phi_{b}^{2}\right) \\
& -\frac{1}{1728}\left(t_{(0)} \phi_{a}^{3}-3 t_{(1)} \phi_{b} \phi_{a}^{2}+3 t_{(2)} \phi_{b}^{2} \phi_{a}-t_{(3)} \phi_{b}^{3}\right)-\frac{1}{12}\left(\lambda_{(0)} \phi_{a}-\lambda_{(1)} \phi_{b}\right) .
\end{aligned}
$$

This mapping also applies if $[\bar{\phi}]$ is ineffective; we simply set $\phi_{a}$ and $\phi_{b}$ to 0 .

With these effects in mind, we now show that one cannot use the charge- 3 construction to successfully obtain eq. (D.1) without an automatic enhancement to $\mathrm{U}(1) \times \mathrm{U}(1)$. We go through the possible choices of divisor classes and demonstrate that they all lead to enhancement or introduce some problem. According to the matter analysis in [36], we should let

$$
[\hat{z}]=-2 K_{B}, \quad\left[\eta_{a}\right] \cdot\left[\eta_{b}\right]=0
$$

to obtain the desired charged hypermultiplet spectrum. This choice implies that

$$
\left[f_{2}\right]=0, \quad[\bar{\phi}]=K_{B}+\left[\eta_{a}\right]+\left[\eta_{b}\right] .
$$

If the model simplifies to Morrison-Park form, $\left[c_{0}\right]=\left[f_{2}\right]$ is trivial, and the divisor classes ensure we see the expected automatic enhancement. We can therefore limit our attention to situations where $\bar{\phi}$ is effective, eliminating many possibilities for $\left[\eta_{a}\right]$ and $\left[\eta_{b}\right]$. When they are both multiples of $F$, for instance, $\bar{\phi}$ is ineffective, and we recover Morrison-Park form. A similar argument shows that this same effect occurs on $\mathbb{F}_{0}$ when both $\left[\eta_{a}\right]$ and $\left[\eta_{b}\right]$ are multiples of $S$. Since the only way to satisfy $\left[\eta_{a}\right] \cdot\left[\eta_{b}\right]=0$ on $\mathbb{F}_{0}$ is to have both $\left[\eta_{a}\right]$ and $\left[\eta_{b}\right]$ be multiples of either $S$ or $F$, we know that automatic enhancement occurs for every possibility on $\mathbb{F}_{0}$, and we can focus on $\mathbb{F}_{n \geq 1}$.

To proceed, it is helpful to write

$$
\left[\eta_{a}\right]=\alpha(S+a F), \quad\left[\eta_{b}\right]=\beta(S+b F)
$$

where $\alpha, \beta$ are positive integers and $a, b$ are non-negative rational numbers such that $\alpha a$, $\beta b$ are integers. ${ }^{28}$ Here, $S$ and $F$ are the divisors on $\mathbb{F}_{n}$ such that $S \cdot S=-n$ and $F \cdot F=0$. In order to have $\left[\eta_{a}\right] \cdot\left[\eta_{b}\right]$ be 0 , we require that

$$
-n+a+b=0
$$

\footnotetext{
${ }^{28}$ If $\left[\eta_{a}\right]$ or $\left[\eta_{b}\right]$ is ineffective, the model reduces to Morrison-Park form (with a different mapping between parameters). It also supports nonabelian gauge factors that can often be Higgsed away. However, these situations do not offer a way of evading the automatic enhancement observed in Morrison-Park form. Additionally, if only one of $\left[\eta_{a}\right]$ or $\left[\eta_{b}\right]$ is proportional to $F,\left[\eta_{a}\right] \cdot\left[\eta_{b}\right]$ is non-zero. The case where both are proportional to $F$ was discussed above.
} 
First, consider cases where $0<a, b<n$. Then, both $\eta_{a}$ and $\eta_{b}$ are reducible, and they share a common factor $v$ with divisor class $S$. We also know that the intersection products

$$
\left[\eta_{a}\right] \cdot S=-\alpha(n-a), \quad\left[\eta_{a}\right] \cdot S=-\beta(n-b)
$$

are negative. And since $\alpha, \beta \geq 1$, the intersection product $[\bar{\phi}] \cdot S$ is negative as well:

$$
\begin{aligned}
{[\bar{\phi}] \cdot S } & =\left(K_{B}+\left[\eta_{a}\right]+\left[\eta_{b}\right]\right) \cdot S \\
& =n-2-\alpha(n-a)-\beta(n-b) \\
& \leq n-2-(n-a)-(n-b)=-2 .
\end{aligned}
$$

Therefore, either $[\bar{\phi}]$ is ineffective - implying the model is in Morrison-Park form—or $\bar{\phi}$ is proportional to $v$-implying that the model has a codimension-one $(4,6)$ singular locus and is invalid.

This leaves the possibility that either $a$ or $b$ is 0 . Without loss of generality, we take $b=0$ and $a=n$. The divisor classes are

$$
\left[\eta_{b}\right]=\beta S, \quad\left[\eta_{a}\right]=\alpha(S+n F),
$$

and $\eta_{b}$ is essentially $v^{\beta}$ with a numerical coefficient. Additionally,

$$
[\bar{\phi}] \cdot S=\left(K_{B}+\left[\eta_{a}\right]+\left[\eta_{b}\right]\right) \cdot S=n+2-\beta n .
$$

Because $\beta$ is positive, this intersection number is negative, implying either that $[\bar{\phi}]$ is ineffective or that $\bar{\phi}$ is proportional to $v^{\beta}$ and hence $\eta_{b}$. Both of these possibilities ensure that the model reduces to Morrison-Park form.

To summarize, if we attempt to use the charge- 3 construction to realize the matter spectrum in eq. (D.1), we either end up with an invalid model or one in Morrison-Park form that exhibits automatic enhancement. This analysis also applies to the charge- 3 construction from [67], which a specialization of the one considered here. Similarly, the charge-4 construction from [36] does not offer a way of realizing this matter spectrum that does not involve the Morrison-Park form. These results support the idea that the automatic enhancement is a genuine physical phenomenon rather than an artifact of the MorrisonPark tuning. Furthermore, since the charge-3 construction relies on the mathematical structure of non-UFD Weierstrass models, this analysis suggests more generally that nonUFD models will not in general produce models that contain only generic charges that can be realized through UFD constructions.

Open Access. This article is distributed under the terms of the Creative Commons Attribution License (CC-BY 4.0), which permits any use, distribution and reproduction in any medium, provided the original author(s) and source are credited.

\section{References}

[1] A. Adams, O. DeWolfe and W. Taylor, String universality in ten dimensions, Phys. Rev. Lett. 105 (2010) 071601 [arXiv:1006.1352] [InSPIRE].

[2] H.-C. Kim, G. Shiu and C. Vafa, Branes and the Swampland, Phys. Rev. D 100 (2019) 066006 [arXiv: 1905.08261] [INSPIRE]. 
[3] M. Montero and C. Vafa, Cobordism Conjecture, Anomalies, and the String Lamppost Principle, JHEP 01 (2021) 063 [arXiv:2008.11729] [INSPIRE].

[4] M. Cvetič, M. Dierigl, L. Lin and H.Y. Zhang, String Universality and Non-Simply-Connected Gauge Groups in 8d, Phys. Rev. Lett. 125 (2020) 211602 [arXiv:2008.10605] [INSPIRE].

[5] C. Vafa, Evidence for F-theory, Nucl. Phys. B 469 (1996) 403 [hep-th/9602022] [InSPIRE].

[6] D.R. Morrison and C. Vafa, Compactifications of F-theory on Calabi-Yau threefolds. 1, Nucl. Phys. B 473 (1996) 74 [hep-th/9602114] [INSPIRE].

[7] D.R. Morrison and C. Vafa, Compactifications of F-theory on Calabi-Yau threefolds. 2, Nucl. Phys. B 476 (1996) 437 [hep-th/9603161] [INSPIRE].

[8] A. Grassi, On minimal models of elliptic threefolds, Math. Ann. 290 (1991) 287.

[9] V. Kumar, D.R. Morrison and W. Taylor, Global aspects of the space of $6 D N=1$ supergravities, JHEP 11 (2010) 118 [arXiv: 1008.1062] [INSPIRE].

[10] W. Taylor, TASI Lectures on Supergravity and String Vacua in Various Dimensions, arXiv:1104.2051 [INSPIRE].

[11] W. Taylor, On the Hodge structure of elliptically fibered Calabi-Yau threefolds, JHEP 08 (2012) 032 [arXiv:1205.0952] [INSPIRE].

[12] N. Seiberg and E. Witten, Comments on string dynamics in six-dimensions, Nucl. Phys. B 471 (1996) 121 [hep-th/9603003] [INSPIRE].

[13] L.B. Anderson, J. Gray, N. Raghuram and W. Taylor, Matter in transition, JHEP 04 (2016) 080 [arXiv: 1512.05791] [INSPIRE].

[14] V. Kumar and W. Taylor, String Universality in Six Dimensions, Adv. Theor. Math. Phys. 15 (2011) 325 [arXiv:0906.0987] [INSPIRE].

[15] C. Vafa, The String landscape and the swampland, hep-th/0509212 [INSPIRE].

[16] E. Palti, The Swampland: Introduction and Review, Fortsch. Phys. 67 (2019) 1900037 [arXiv: 1903.06239] [INSPIRE].

[17] N. Raghuram, W. Taylor and A.P. Turner, General F-theory models with tuned $(\mathrm{SU}(3) \times \mathrm{SU}(2) \times \mathrm{U}(1)) / \mathbb{Z}_{6}$ symmetry, JHEP 04 (2020) 008 [arXiv:1912.10991] [INSPIRE].

[18] D.R. Morrison and D.S. Park, F-Theory and the Mordell-Weil Group of Elliptically-Fibered Calabi-Yau Threefolds, JHEP 10 (2012) 128 [arXiv:1208.2695] [INSPIRE].

[19] D.R. Morrison and W. Taylor, Charge completeness and the massless charge lattice in f-theory models of supergravity, to appear.

[20] S. Cecotti, C. Cordova, J.J. Heckman and C. Vafa, T-Branes and Monodromy, JHEP 07 (2011) 030 [arXiv: 1010.5780] [INSPIRE].

[21] L.B. Anderson, J.J. Heckman and S. Katz, T-Branes and Geometry, JHEP 05 (2014) 080 [arXiv: 1310.1931] [INSPIRE].

[22] A. Sagnotti, A Note on the Green-Schwarz mechanism in open string theories, Phys. Lett. B 294 (1992) 196 [hep-th/9210127] [INSPIRE].

[23] M.B. Green, J.H. Schwarz and P.C. West, Anomaly Free Chiral Theories in Six-Dimensions, Nucl. Phys. B 254 (1985) 327 [INSPIRE]. 
[24] D.S. Park and W. Taylor, Constraints on 6D Supergravity Theories with Abelian Gauge Symmetry, JHEP 01 (2012) 141 [arXiv:1110.5916] [INSPIRE].

[25] W. Taylor and A.P. Turner, An infinite swampland of U(1) charge spectra in 6D supergravity theories, JHEP 06 (2018) 010 [arXiv: 1803.04447] [INSPIRE].

[26] T. Weigand, F-theory, PoS TASI2017 (2018) 016 [arXiv:1806.01854] [InSPIRE].

[27] V. Kumar, D.S. Park and W. Taylor, 6D supergravity without tensor multiplets, JHEP 04 (2011) 080 [arXiv: 1011.0726] [INSPIRE].

[28] D. Klevers, D.R. Morrison, N. Raghuram and W. Taylor, Exotic matter on singular divisors in F-theory, JHEP 11 (2017) 124 [arXiv:1706.08194] [INSPIRE].

[29] W. Taylor and A.P. Turner, Generic matter representations in 6D supergravity theories, JHEP 05 (2019) 081 [arXiv:1901.02012] [INSPIRE].

[30] M. Bershadsky, K.A. Intriligator, S. Kachru, D.R. Morrison, V. Sadov and C. Vafa, Geometric singularities and enhanced gauge symmetries, Nucl. Phys. B 481 (1996) 215 [hep-th/9605200] [INSPIRE].

[31] V. Kumar and W. Taylor, A Bound on 6D N=1 supergravities, JHEP 12 (2009) 050 [arXiv: 0910.1586] [INSPIRE].

[32] J.H. Schwarz, Anomaly-free supersymmetric models in six-dimensions, Phys. Lett. B 371 (1996) 223 [hep-th/9512053] [INSPIRE].

[33] N. Seiberg and W. Taylor, Charge Lattices and Consistency of 6D Supergravity, JHEP 06 (2011) 001 [arXiv:1103.0019] [INSPIRE].

[34] S. Monnier, G.W. Moore and D.S. Park, Quantization of anomaly coefficients in $6 D$ $\mathcal{N}=(1,0)$ supergravity, JHEP 02 (2018) 020 [arXiv:1711.04777] [INSPIRE].

[35] S. Monnier and G.W. Moore, Remarks on the Green-Schwarz Terms of Six-Dimensional Supergravity Theories, Commun. Math. Phys. 372 (2019) 963 [arXiv:1808.01334] [INSPIRE].

[36] N. Raghuram, Abelian F-theory Models with Charge-3 and Charge-4 Matter, JHEP 05 (2018) 050 [arXiv: 1711.03210] [INSPIRE].

[37] L. Bhardwaj, D.R. Morrison, Y. Tachikawa and A. Tomasiello, The frozen phase of F-theory, JHEP 08 (2018) 138 [arXiv:1805.09070] [INSPIRE].

[38] T. Banks and N. Seiberg, Symmetries and Strings in Field Theory and Gravity, Phys. Rev. D 83 (2011) 084019 [arXiv: 1011.5120] [INSPIRE].

[39] D. Harlow and H. Ooguri, Symmetries in quantum field theory and quantum gravity, Commun. Math. Phys. 383 (2021) 1669 [arXiv: 1810.05338] [INSPIRE].

[40] T. Banks, N. Seiberg and E. Silverstein, Zero and one-dimensional probes with $N=8$ supersymmetry, Phys. Lett. B 401 (1997) 30 [hep-th/9703052] [INSPIRE].

[41] S.-J. Lee and T. Weigand, Swampland Bounds on the Abelian Gauge Sector, Phys. Rev. D 100 (2019) 026015 [arXiv: 1905.13213] [INSPIRE].

[42] D.R. Morrison and W. Taylor, Matter and singularities, JHEP 01 (2012) 022 [arXiv: 1106.3563] [INSPIRE].

[43] S.B. Johnson and W. Taylor, Enhanced gauge symmetry in 6D F-theory models and tuned elliptic Calabi-Yau threefolds, Fortsch. Phys. 64 (2016) 581 [arXiv:1605.08052] [inSPIRE]. 
[44] D.R. Morrison and W. Taylor, Classifying bases for 6D F-theory models, Central Eur. J. Phys. 10 (2012) 1072 [arXiv:1201.1943] [INSPIRE].

[45] B. Haghighat, A. Klemm, G. Lockhart and C. Vafa, Strings of Minimal 6d SCFTs, Fortsch. Phys. 63 (2015) 294 [arXiv:1412.3152] [INSPIRE].

[46] H.-C. Kim, S. Kim and J. Park, 6d strings from new chiral gauge theories, arXiv: 1608.03919 [INSPIRE].

[47] H. Shimizu and Y. Tachikawa, Anomaly of strings of $6 d \mathcal{N}=(1,0)$ theories, JHEP 11 (2016) 165 [arXiv: 1608.05894] [INSPIRE].

[48] A. Grassi and D.R. Morrison, Anomalies and the Euler characteristic of elliptic Calabi-Yau threefolds, Commun. Num. Theor. Phys. 6 (2012) 51 [arXiv:1109.0042] [INSPIRE].

[49] Y.-C. Huang and W. Taylor, Comparing elliptic and toric hypersurface Calabi-Yau threefolds at large Hodge numbers, JHEP 02 (2019) 087 [arXiv: 1805.05907] [INSPIRE].

[50] N. Seiberg, Nontrivial fixed points of the renormalization group in six-dimensions, Phys. Lett. B 390 (1997) 169 [hep-th/9609161] [INSPIRE].

[51] J.J. Heckman, D.R. Morrison and C. Vafa, On the Classification of 6D SCFTs and Generalized ADE Orbifolds, JHEP 05 (2014) 028 [Erratum ibid. 06 (2015) 017] [arXiv: 1312.5746] [INSPIRE].

[52] M. Del Zotto, J.J. Heckman, A. Tomasiello and C. Vafa, 6d Conformal Matter, JHEP 02 (2015) 054 [arXiv: 1407.6359] [INSPIRE].

[53] M. Cvetič, J.J. Heckman and L. Lin, Towards Exotic Matter and Discrete Non-Abelian Symmetries in F-theory, JHEP 11 (2018) 001 [arXiv:1806.10594] [INSPIRE].

[54] S. Katz, D.R. Morrison, S. Schäfer-Nameki and J. Sully, Tate's algorithm and F-theory, JHEP 08 (2011) 094 [arXiv: 1106.3854] [INSPIRE].

[55] S.H. Katz and C. Vafa, Matter from geometry, Nucl. Phys. B 497 (1997) 146 [hep-th/9606086] [INSPIRE].

[56] N. Raghuram and A.P. Turner, Orders of vanishing and u(1) charges in f-theory, to appear.

[57] F. Baume, M. Cvetič, C. Lawrie and L. Lin, When rational sections become cyclic - Gauge enhancement in F-theory via Mordell-Weil torsion, JHEP 03 (2018) 069 [arXiv: 1709.07453] [INSPIRE].

[58] L.-F. Li, Group Theory of the Spontaneously Broken Gauge Symmetries, Phys. Rev. D 9 (1974) 1723 [INSPIRE].

[59] U.H. Danielsson and P. Stjernberg, Notes on equivalences and Higgs branches in $N=2$ supersymmetric Yang-Mills theory, Phys. Lett. B 380 (1996) 68 [hep-th/9603082] [INSPIRE].

[60] Y.-N. Wang, Tuned and Non-Higgsable U(1)s in F-theory, JHEP 03 (2017) 140 [arXiv: 1611.08665] [INSPIRE].

[61] D.R. Morrison and W. Taylor, Sections, multisections, and U(1) fields in F-theory, arXiv: 1404.1527 [INSPIRE].

[62] H. Tarazi and C. Vafa, On finiteness of supergravity landscapes in $d=6$, to appear.

[63] N. Raghuram and W. Taylor, Large U(1) charges in F-theory, JHEP 10 (2018) 182 [arXiv: 1809.01666] [INSPIRE]. 
[64] F.M. Cianci, D.K. Mayorga Peña and R. Valandro, High U(1) charges in type IIB models and their F-theory lift, JHEP 04 (2019) 012 [arXiv:1811.11777] [INSPIRE].

[65] A. Collinucci, M. Fazzi, D.R. Morrison and R. Valandro, High electric charges in M-theory from quiver varieties, JHEP 11 (2019) 111 [arXiv:1906.02202] [INSPIRE].

[66] L.B. Anderson, J. Gray and P.-K. Oehlmann, F-Theory on Quotients of Elliptic Calabi-Yau Threefolds, JHEP 12 (2019) 131 [arXiv:1906.11955] [INSPIRE].

[67] D. Klevers, D.K. Mayorga Peña, P.-K. Oehlmann, H. Piragua and J. Reuter, F-Theory on all Toric Hypersurface Fibrations and its Higgs Branches, JHEP 01 (2015) 142 [arXiv: 1408.4808] [INSPIRE].

[68] D. Klevers and W. Taylor, Three-Index Symmetric Matter Representations of SU(2) in F-theory from Non-Tate Form Weierstrass Models, JHEP 06 (2016) 171 [arXiv: 1604.01030] [INSPIRE].

[69] J. Tian and Y.-N. Wang, E-string spectrum and typical F-theory geometry, arXiv: 1811.02837 [INSPIRE].

[70] V. Braun and D.R. Morrison, F-theory on Genus-One Fibrations, JHEP 08 (2014) 132 [arXiv: 1401.7844] [INSPIRE].

[71] M. Cvetič and L. Lin, The Global Gauge Group Structure of F-theory Compactification with $\mathrm{U}(1) s, J H E P 01$ (2018) 157 [arXiv:1706.08521] [INSPIRE].

[72] M. Cvetič, D. Klevers and H. Piragua, F-Theory Compactifications with Multiple U(1)-Factors: Constructing Elliptic Fibrations with Rational Sections, JHEP 06 (2013) 067 [arXiv: 1303.6970] [INSPIRE].

[73] M. Cvetič, D. Klevers, H. Piragua and W. Taylor, General U(1) $\times \mathrm{U}(1)$ F-theory compactifications and beyond: geometry of unHiggsings and novel matter structure, JHEP 11 (2015) 204 [arXiv: 1507.05954] [inSPIRE].

[74] W. Taylor and Y.-N. Wang, Non-toric bases for elliptic Calabi-Yau threefolds and 6D F-theory vacua, Adv. Theor. Math. Phys. 21 (2017) 1063 [arXiv:1504.07689] [InSPIRE]. 